\title{
RERTR-7 Irradiation Summary Report
}

D. M. Perez

M. A. Lillo

G. S. Chang

G. A. Roth

N. E. Woolstenhulme

D. M. Wachs

December 2011

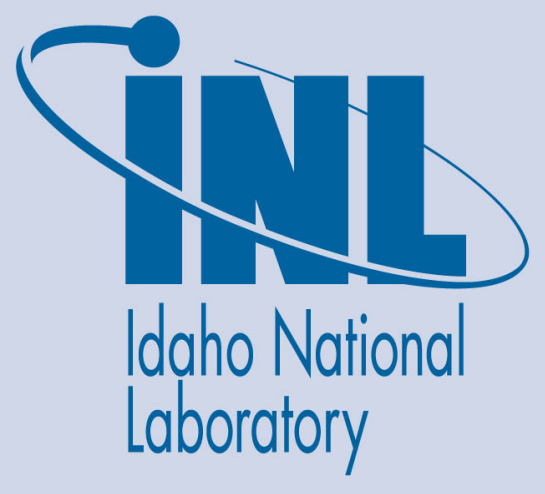

The INL is a U.S. Department of Energy National Laboratory operated by Battelle Energy Alliance 


\section{DISCLAIMER}

This information was prepared as an account of work sponsored by an agency of the U.S. Government. Neither the U.S. Government nor any agency thereof, nor any of their employees, makes any warranty, expressed or implied, or assumes any legal liability or responsibility for the accuracy, completeness, or usefulness, of any information, apparatus, product, or process disclosed, or represents that its use would not infringe privately owned rights. References herein to any specific commercial product, process, or service by trade name, trade mark, manufacturer, or otherwise, does not necessarily constitute or imply its endorsement, recommendation, or favoring by the U.S. Government or any agency thereof. The views and opinions of authors expressed herein do not necessarily state or reflect those of the U.S. Government or any agency thereof. 


\title{
RERTR-7 Irradiation Summary Report
}

\author{
D. M. Perez \\ M. A. Lillo \\ G. S. Chang \\ G. A. Roth \\ N. E. Woolstenhulme \\ D. M. Wachs
}

December 2011

\author{
Idaho National Laboratory \\ Fuel Performance and Design \\ Idaho Falls, Idaho 83415
}

http://www.inl.gov

\author{
Prepared for the \\ U.S. Department of Energy \\ Office of National Nuclear Security Administration \\ Under DOE Idaho Operations Office \\ Contract DE-AC07-05ID14517
}



RERTR-7 Irradiation Summary Report

INL/EXT-11-24283

Revision 0

December 2011

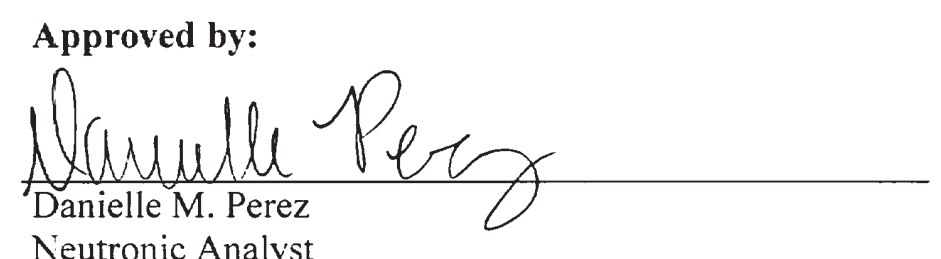

Neutronic Analyst

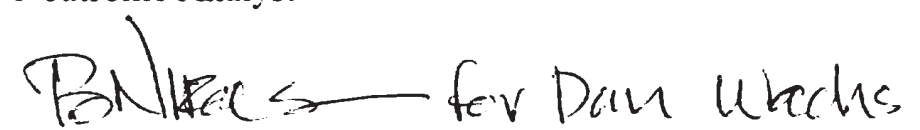

Daniel M. Wacks

Principle Investigator

PAl

Bruce Nelson

Experiment Manager
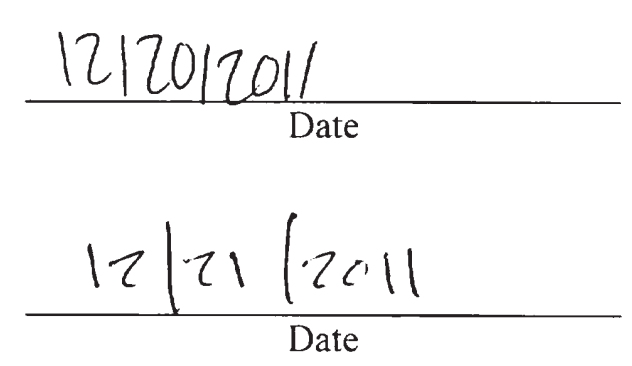

$\frac{12 / 20 \mid 2011}{\text { Date }}$ 


\section{SUMMARY}

The Reduced Enrichment for Research and Test Reactor (RERTR) experiment RERTR-7A, was designed to test several modified fuel designs to target fission densities representative of a peak low enriched uranium (LEU) burnup in excess of 90\% U-235 at peak experiment power sufficient to generate a peak surface heat flux of approximately $300 \mathrm{~W} / \mathrm{cm}^{2}$. The RERTR-7B experiment was designed as a high power test of 'second generation' dispersion fuels at peak experiment power sufficient to generate a surface heat flux on the order of 230 $\mathrm{W} / \mathrm{cm}^{2}{ }^{1}$

The following report summarizes the life of the RERTR-7A and RERTR-7B experiments through end of irradiation, including as-run neutronic analyses, thermal analyses and hydraulic testing results. 


\section{CONTENTS}

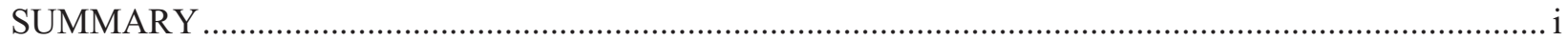

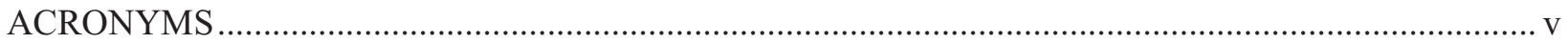

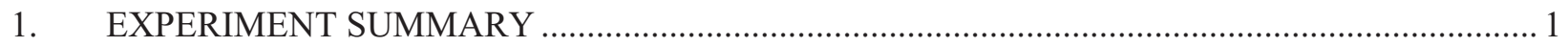

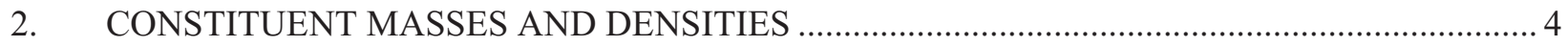

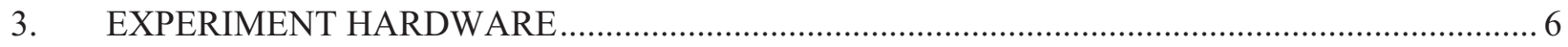

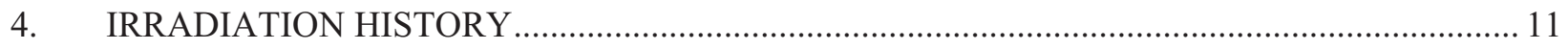

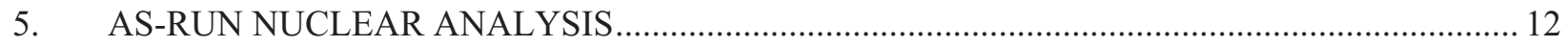

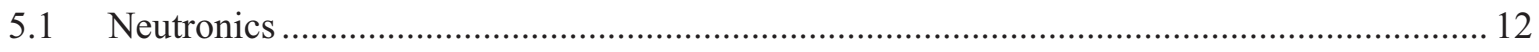

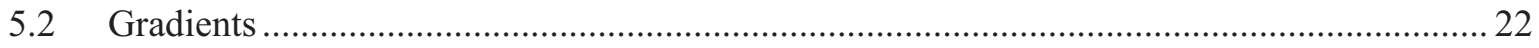

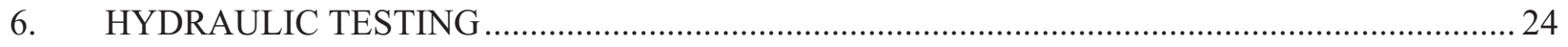

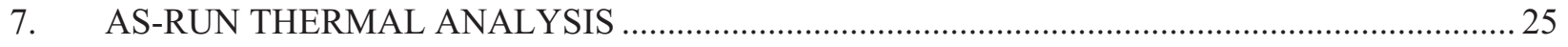

7.1 Coolant Temperature as a Function of Location ................................................................ 25

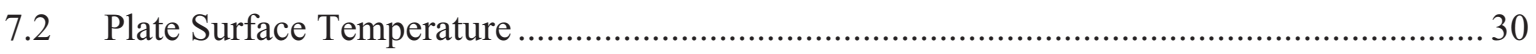

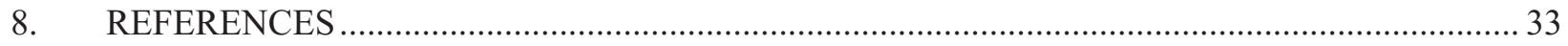

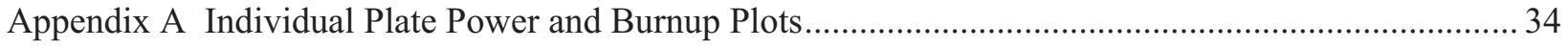

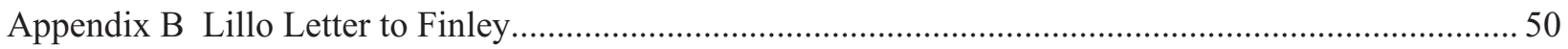

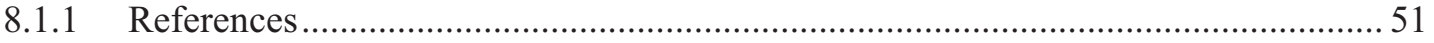

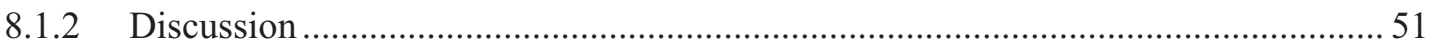

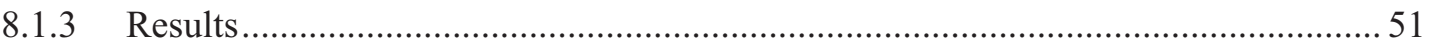

\section{FIGURES}

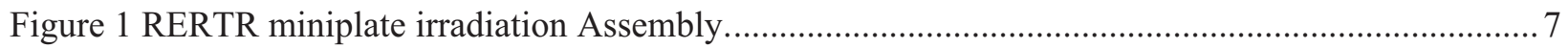

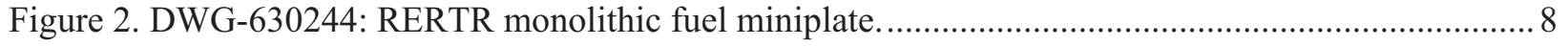

Figure 3. DWG-630238: RERTR dispersion fuel miniplate..............................................................

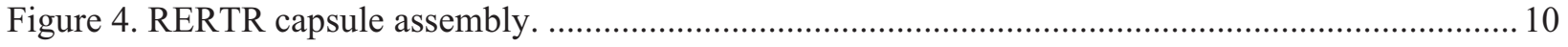

Figure 5. RERTR-7A fuel miniplates thermal neutron flux L2ARs in transverse direction. ${ }^{6}$.................... 22

Figure 6. RERTR-7A fuel miniplates fission rate L2ARs in transverse direction. ${ }^{6}$................................23

Figure 7. RERTR-7A coolant temperature as a function of location along the test assembly for

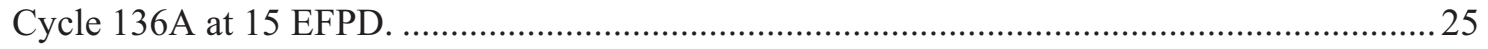

Figure 8. RERTR-7A coolant temperature as a function of location along the test assembly for Cycle 136A at 18 EFPD. 
Figure 9. RERTR-7A coolant temperature as a function of location along the test assembly for Cycle 136A at 50.9 EFPD.

Figure 10. RERTR-7A coolant temperature as a function of location along the test assembly for Cycle $146 \mathrm{~B}$ at 12 EFPD.

Figure 11. RERTR-7A coolant temperature as a function of location along the test assembly for Cycle 146B at 25.5 EFPD.

Figure 12. RERTR-7A coolant temperature as a function of location along the test assembly for Cycle $146 \mathrm{~B}$ at 39 EFPD 28

Figure 13. RERTR-7B coolant temperature as a function of location along the test assembly for Cycle $146 \mathrm{~B}$ at 15 EFPD .28

Figure 14. RERTR-7B coolant temperature as a function of location along the test assembly for Cycle $146 \mathrm{~B}$ at 30 EFPD.

Figure 15. RERTR-7B coolant temperature as a function of location along the test assembly for Cycle $146 \mathrm{~B}$ at 39 EFPD.

\section{TABLES}

Table 1. RERTR-7A experiment matrix loading diagram. ${ }^{1}$................................................................. 2

Table 2. RERTR-7B experiment matrix loading diagram. ${ }^{1}$.............................................................. 3

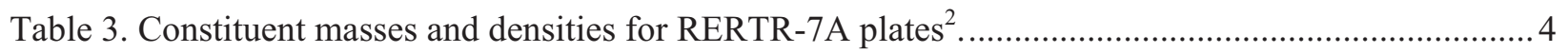

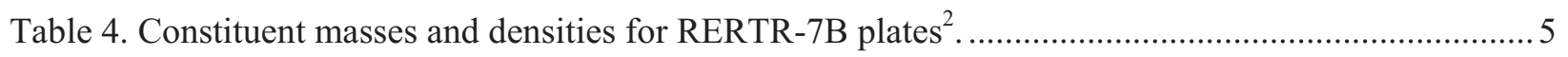

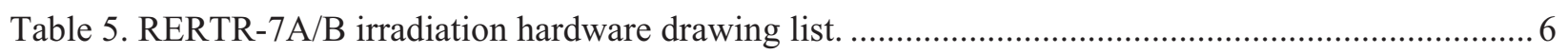

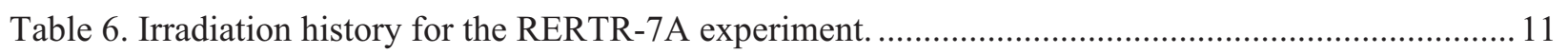

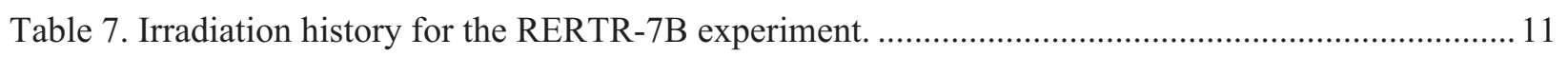

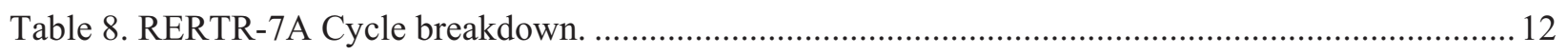

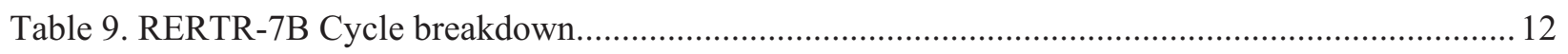

Table 10. RERTR-7A average plate power and burnup for MOC1 136A (15 EFPD)............................. 13

Table 11. RERTR-7A average plate power and burnup for MOC2 136A (35 EFPD)............................ 14

Table 12. RERTR-7A average plate power and burnup for EOC 136A (50.9 EFPD). ........................... 15

Table 13. RERTR-7A average plate power and burnup for MOC1 136B (12.0 EFPD, 62.9 EFPD

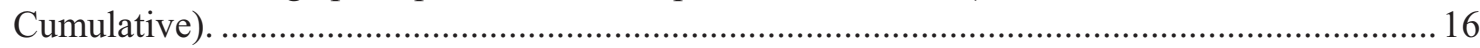

Table 14. RERTR-7A average plate power and burnup for MOC2 136B (25.5 EFPD, 76.4 EFPD Cumulative).

Table 15. RERTR-7A average plate power and burnup for EOC 136B (39 EFPD, 89.9 EFPD Cumulative). 18

Table 16. RERTR-7B average plate power and burnup for MOC1 136B (15 EFPD)............................. 19

Table 17. RERTR-7B average plate power and burnup for MOC2 136B (30 EFPD)..............................20

Table 18. RERTR-7B average plate power and burnup for EOC 136B (39 EFPD)..............................2 21

Table 19. Loss coefficients for the RERTR irradiation test vehicle components. ${ }^{7}$.................................. 24 
Table 20. RERTR-7A plate surface temperatures for Cycle 136A (50.9 EFPD).

Table 21. RERTR-7A plate surface temperatures for Cycle 136B (39 EFPD)....................................... 31

Table 22. RERTR-7B plate surface temperatures for Cycle 136B (39 EFPD)....................................... 32 


\section{ACRONYMS}

Al aluminum

ATR Advanced Test Reactor

CNEA Comisión Nacional de Energía Atómica (The National Atomic Enerty Commission) Argentina

EFPD effective full power days

FSW friction stir weld

L2AR local-to-average ratio

LEU low enriched uranium

MCNP Monte Carlo N-Particle

Mo molybdenum

RERTR Reduced Enrichment Research and Test Reactor

Si silicon

Ti titanium

TLPB transient liquid phase bonding

TS thermal spray

U uranium

U-Mo uranium-molybdenum

$\mathrm{Zr} \quad$ zirconium 


\section{RERTR-7 Irradiation Summary Report \\ 1. EXPERIMENT SUMMARY}

In support of the Global Threat Reduction Initiative (GTRI) Fuel Development (FD) program

(historically known as Reduced Enrichment for Research and Test Reactors (RERTR)), the RERTR-7A

experiment was designed to test several modified fuel designs to target fission densities representative of a peak low enriched uranium (LEU) burnup in excess of $90 \%$ U-235 at peak experiment power sufficient to generate a peak surface heat flux of approximately $300 \mathrm{~W} / \mathrm{cm}^{2}$. The RERTR-7B experiment was designed as a high power test of 'second generation' dispersion fuels at peak experiment power sufficient to generate a surface heat flux on the order of $230 \mathrm{~W} / \mathrm{cm}^{2}$.

The RERTR-8 test assembly holds 4 capsules, designated as A, B, C and D, with A at the top of the assembly and D at the bottom. Each capsule has 2 levels, with 4 plate positions per level, for a total of 8 plate positions per capsule and 32 plate positions per assembly. Within each capsule the 8 plate positions are azimuthally designated as 1 through 4 in the upper level and 5 through 8 in the lower level. The loading diagram for the RERTR-8 experiment matrix is shown in Table 1. 
Table 1. RERTR-7A experiment matrix loading diagram. ${ }^{1}$

\begin{tabular}{|c|c|c|c|c|}
\hline \multicolumn{5}{|c|}{ RERTR-7A Experiment Matrix } \\
\hline Capsule & Column 1 & Column 2 & Column 3 & Column 4 \\
\hline \multirow[b]{2}{*}{$\begin{array}{c}\text { A } \\
\text { Top }\end{array}$} & A1 & A2 & A3 & A4 \\
\hline & $\begin{array}{l}\text { DUM11 } \\
\text { BLANK }\end{array}$ & $\begin{array}{l}\text { DUM14 } \\
\text { BLANK }\end{array}$ & $\begin{array}{l}\text { DUM12 } \\
\text { BLANK }\end{array}$ & $\begin{array}{c}\text { DUM8 } \\
\text { BLANK }\end{array}$ \\
\hline \multirow{4}{*}{$\underset{\text { Bottom }}{\text { A }}$} & A5 & A6 & A7 & A8 \\
\hline & R3R040 & V5R040 & R5R030 & H1F020 \\
\hline & U-7Mo Roll & U-7Mo Roll & U-10Mo Roll & U-12Mo FSW \\
\hline & Al 4043 Matrix & Al-0.5Si Matrix & Al-0.5 Si Matrix & 0.010" Foil \\
\hline \multirow{4}{*}{$\begin{array}{c}\text { B } \\
\text { Top }\end{array}$} & B1 & $\mathrm{B} 2$ & B3 & B4 \\
\hline & R1R040 & R2R040 & R0R010 & H1T010 \\
\hline & U-7Mo Roll & U-7Mo Roll & U-7Mo Roll & U-12Mo TLPB \\
\hline & Al 6061 Matrix & Al-2Si Matrix & Pure Al Matrix & 0.010" Foil \\
\hline \multirow{4}{*}{$\begin{array}{c}\text { B } \\
\text { Bottom }\end{array}$} & B5 & B6 & B7 & B8 \\
\hline & L1F01L & V5R050 & L1F140 & MZ25 \\
\hline & U-10Mo FSW & U-7Mo Roll & U-10Mo FSW & U-7Mo Roll \\
\hline & Holed Foil & Al-0.5Si Matrix & 0.010" Foil & Zr Clad CNEA \\
\hline \multirow{4}{*}{$\begin{array}{c}\text { C } \\
\text { Top }\end{array}$} & $\mathrm{C} 1$ & $\mathrm{C} 2$ & $\mathrm{C} 3$ & $\mathrm{C} 4$ \\
\hline & H1F030 & L1T020 & L1F1 10 & MZ50 \\
\hline & U-12Mo FSW & U-10Mo TLPB & U-10Mo FSW & U-7Mo Roll \\
\hline & 0.010" Foil & 0.010" Foil & 0.010" Foil & Zr Clad CNEA \\
\hline \multirow{4}{*}{$\begin{array}{c}\text { C } \\
\text { Bottom }\end{array}$} & $\mathrm{C} 5$ & C6 & $\mathrm{C} 7$ & $\mathrm{C} 8$ \\
\hline & L1F120 & H1Т020 & R3R050 & R5R040 \\
\hline & U-10Mo FSW & U-12Mo TLPB & U-7Mo Roll & U-10Mo Roll \\
\hline & 0.010" Foil & $0.010 "$ Foil & Al 4043 Matrix & Al-0.5Si Matrix \\
\hline \multirow{3}{*}{$\begin{array}{c}\text { D } \\
\text { Top }\end{array}$} & D1 & D2 & D3 & D4 \\
\hline & R1R050 & DUM13 & R0R020 & DUM19 \\
\hline & $\begin{array}{c}\text { U-7Mo Roll } \\
\text { Al } 6061 \text { Matrix }\end{array}$ & BLANK & $\begin{array}{c}\text { U-7Mo Roll } \\
\text { Pure Al Matrix }\end{array}$ & BLANK \\
\hline \multirow{4}{*}{$\underset{\text { Bottom }}{\text { D }}$} & D5 & D6 & D7 & D8 \\
\hline & DUM05 & L1F160 & L2F040 & R2R050 \\
\hline & BLANK & U-10Mo FSW & U-10Mo TLPB & U-7Mo Roll \\
\hline & & 0.010" Foil & $0.020 "$ Foil & Al-2Si Matrix \\
\hline
\end{tabular}


Table 2. RERTR-7B experiment matrix loading diagram. ${ }^{1}$

\begin{tabular}{|c|c|c|c|c|}
\hline \multicolumn{5}{|c|}{ RERTR-7B Experiment Matrix } \\
\hline Capsule & Column 1 & Column 2 & Column 3 & Column 4 \\
\hline A & A1 & A2 & A3 & A4 \\
Top & BLANK & BLANK & BLANK & BLANK \\
\hline A & A5 & A6 & A7 & A8 \\
Bottom & BLANK & BLANK & BLANK & BLANK \\
\hline B & B1 & B2 & B3 & B4 \\
Top & BLANK & BLANK & BLANK & BLANK \\
\hline B & B5 & B6 & B7 & B8 \\
Bottom & BLANK & BLANK & BLANK & BLANK \\
\hline & C1 & C2 & C3 & C4 \\
C & F3R010 & R0R010 & R3R010 & D3R010 \\
Top & U-7Mo-2Zr Roll & U-7Mo & U-7Mo & U-7Mo-1Ti Roll \\
& A1 4043 Matrix & A1 Matrix & Al 4043 Matrix & Al 4043 Matrix \\
\hline C & C5 & C6 & C7 & C8 \\
Bottom & BLANK & BLANK & BLANK & BLANK \\
\hline D & D1 & D2 & D3 & D4 \\
Top & BLANK & BLANK & BLANK & BLANK \\
\hline D & D5 & D6 & D7 & D8 \\
Bottom & BLANK & BLANK & BLANK & BLANK \\
\hline
\end{tabular}




\section{CONSTITUENT MASSES AND DENSITIES}

The constituent masses and densities for the RERTR-7A and -7B plates were obtained from the asbuilt plate summary sheets. Table 3 summarizes the constituent masses and densities for the plates in the RERTR-7A experiment and Table 4 summarizes the constituent masses and densities for the plates in the RERTR-7B experiment.

Table 3. Constituent masses and densities for RERTR-7A plates ${ }^{2}$.

\begin{tabular}{|c|c|c|c|c|c|c|c|c|c|}
\hline \multirow{2}{*}{$\begin{array}{c}\text { Fuel } \\
\text { Plate } \\
\text { ID } \\
\end{array}$} & \multirow[b]{2}{*}{$\begin{array}{c}\text { Fuel } \\
\text { Plate \# }\end{array}$} & \multicolumn{4}{|c|}{ Fuel Constituent Masses } & \multicolumn{4}{|c|}{ Constituent Densities } \\
\hline & & $\begin{array}{c}\text { Total-U } \\
(\mathrm{g})\end{array}$ & $\begin{array}{c}\text { U-235 } \\
(\mathrm{g})\end{array}$ & $\begin{array}{l}\text { Mo } \\
(\mathrm{g})\end{array}$ & $\begin{array}{c}\text { Matrix } \\
(\mathrm{g})\end{array}$ & $\begin{array}{c}\text { Total-U } \\
(\mathrm{g} / \mathrm{cc})\end{array}$ & $\begin{array}{l}\text { U-235 } \\
(\mathrm{g} / \mathrm{cc})\end{array}$ & $\begin{array}{c}\text { Mo } \\
(\mathrm{g} / \mathrm{cc})\end{array}$ & $\begin{array}{c}\text { Matrix } \\
(\mathrm{g} / \mathrm{cc})\end{array}$ \\
\hline $\mathrm{A} 1$ & Blank & -- & -- & -- & -- & -- & -- & -- & -- \\
\hline $\mathrm{A} 2$ & Blank & -- & -- & -- & -- & -- & -- & -- & -- \\
\hline A3 & Blank & -- & -- & -- & -- & -- & -- & -- & -- \\
\hline A4 & Blank & -- & -- & -- & -- & -- & -- & -- & -- \\
\hline A5 & R3R040 & 6.060 & 3.530 & 0.459 & 1.431 & 6.809 & 3.966 & 0.516 & 1.608 \\
\hline A6 & V5R040 & 6.130 & 3.550 & 0.681 & 1.369 & 6.811 & 3.944 & 0.757 & 1.521 \\
\hline A7 & R5R030 & 6.080 & 3.540 & 0.458 & 1.422 & 6.909 & 4.023 & 0.520 & 1.616 \\
\hline $\mathrm{A} 8$ & $\mathrm{H} 1 \mathrm{~F} 020$ & 5.650 & 3.330 & 0.780 & -- & 14.487 & 8.538 & 2.000 & -- \\
\hline B1 & R1R040 & 6.120 & 3.550 & 0.460 & 1.380 & 7.116 & 4.128 & 0.535 & 1.605 \\
\hline $\mathrm{B} 2$ & R2R040 & 6.070 & 3.530 & 0.460 & 1.420 & 6.670 & 3.879 & 0.505 & 1.560 \\
\hline B3 & R0R010 & 6.080 & 3.530 & 0.448 & 1.422 & 6.909 & 4.011 & 0.509 & 1.616 \\
\hline B4 & H1T010 & 5.520 & 3.210 & 0.750 & -- & 14.526 & 8.447 & 1.974 & -- \\
\hline B5 & L1F01L & 5.300 & 3.160 & 0.600 & -- & 15.143 & 9.029 & 1.714 & -- \\
\hline B6 & V5R050 & 6.100 & 3.540 & 0.678 & 1.372 & 6.703 & 3.890 & 0.745 & 1.508 \\
\hline B7 & L1F140 & 5.860 & 3.410 & 0.660 & -- & 15.421 & 8.974 & 1.737 & -- \\
\hline $\mathrm{B} 8$ & $\mathrm{MZ} 25^{*}$ & 5.900 & 1.170 & 0.450 & -- & 14.861 & 2.947 & 1.134 & -- \\
\hline $\mathrm{C} 1$ & H1F030 & 5.820 & 3.380 & 0.800 & -- & 14.550 & 8.450 & 2.000 & -- \\
\hline $\mathrm{C} 2$ & L1T020 & 5.740 & 3.420 & 0.650 & -- & 15.105 & 9.000 & 1.711 & -- \\
\hline $\mathrm{C} 3$ & L1F110 & 5.910 & 3.440 & 0.660 & -- & 15.154 & 8.821 & 1.692 & -- \\
\hline $\mathrm{C} 4$ & MZ 50* & 10.900 & 2.160 & 1.000 & -- & 13.728 & 2.720 & 1.259 & -- \\
\hline $\mathrm{C} 5$ & L1F120 & 5.960 & 3.470 & 0.660 & -- & 15.282 & 8.897 & 1.692 & -- \\
\hline C6 & Н1T020 & 5.400 & 3.190 & 0.750 & -- & 14.595 & 8.622 & 2.027 & -- \\
\hline $\mathrm{C} 7$ & R3R050 & 6.080 & 3.540 & 0.458 & 1.432 & 6.909 & 4.023 & 0.520 & 1.627 \\
\hline $\mathrm{C} 8$ & R5R040 & 6.070 & 3.540 & 0.464 & 1.426 & 6.898 & 4.023 & 0.527 & 1.620 \\
\hline D1 & R1R050 & 6.080 & 3.540 & 0.460 & 1.380 & 7.070 & 4.116 & 0.535 & 1.605 \\
\hline $\mathrm{D} 2$ & Blank & -- & -- & -- & -- & -- & -- & -- & -- \\
\hline D3 & R0R020 & 6.080 & 3.530 & 0.450 & 1.420 & 6.831 & 3.966 & 0.506 & 1.596 \\
\hline D4 & Blank & -- & -- & -- & -- & -- & -- & -- & -- \\
\hline D5 & Blank & -- & -- & -- & -- & -- & -- & -- & -- \\
\hline D6 & L1F160 & 4.870 & 2.830 & 0.540 & -- & 15.219 & 8.844 & 1.688 & -- \\
\hline D7 & L2F040 & 10.820 & 6.310 & 1.220 & -- & 15.457 & 9.014 & 1.743 & -- \\
\hline D8 & R2R050 & 6.070 & 3.530 & 0.462 & 1.418 & 6.898 & 4.011 & 0.525 & 1.611 \\
\hline
\end{tabular}


Table 4. Constituent masses and densities for RERTR-7B plates ${ }^{2}$.

\begin{tabular}{|c|c|c|c|c|c|c|c|c|c|c|c|}
\hline \multirow{2}{*}{$\begin{array}{c}\text { Fuel } \\
\text { Plate } \\
\text { ID } \\
\end{array}$} & \multirow[b]{2}{*}{$\begin{array}{c}\text { Fuel } \\
\text { Plate \# }\end{array}$} & \multicolumn{5}{|c|}{ Fuel Constituent Masses } & \multicolumn{5}{|c|}{ Constituent Densities } \\
\hline & & $\begin{array}{c}\text { Total-U } \\
(\mathrm{g})\end{array}$ & $\begin{array}{c}\text { U-235 } \\
(\mathrm{g}) \\
\end{array}$ & $\begin{array}{l}\text { Mo } \\
(\mathrm{g})\end{array}$ & $\begin{array}{c}\mathrm{Zr} / \mathrm{Ti} \\
(\mathrm{g})\end{array}$ & $\begin{array}{c}\text { Matrix } \\
(\mathrm{g})\end{array}$ & $\begin{array}{c}\text { Total-U } \\
(\mathrm{g} / \mathrm{cc})\end{array}$ & $\begin{array}{l}\text { U-235 } \\
(\mathrm{g} / \mathrm{cc})\end{array}$ & $\begin{array}{c}\text { Mo } \\
(\mathrm{g} / \mathrm{cc})\end{array}$ & $\begin{array}{l}\mathrm{Zr} / \mathrm{Ti} \\
(\mathrm{g} / \mathrm{cc})\end{array}$ & $\begin{array}{c}\text { Matrix } \\
(\mathrm{g} / \mathrm{cc})\end{array}$ \\
\hline $\mathrm{A} 1$ & Blank & -- & -- & -- & -- & -- & -- & -- & -- & -- & -- \\
\hline $\mathrm{A} 2$ & Blank & -- & -- & -- & -- & -- & -- & -- & -- & -- & -- \\
\hline A3 & Blank & -- & -- & -- & -- & -- & -- & -- & -- & -- & -- \\
\hline $\mathrm{A} 4$ & Blank & -- & -- & -- & -- & -- & -- & -- & -- & -- & -- \\
\hline A5 & Blank & -- & -- & -- & -- & -- & -- & -- & -- & -- & -- \\
\hline A6 & Blank & -- & -- & -- & -- & -- & -- & -- & -- & -- & -- \\
\hline A7 & Blank & -- & -- & -- & -- & -- & -- & -- & -- & -- & -- \\
\hline A8 & Blank & -- & -- & -- & -- & -- & -- & -- & -- & -- & -- \\
\hline $\mathrm{B} 1$ & Blank & -- & -- & -- & -- & -- & -- & -- & -- & -- & -- \\
\hline $\mathrm{B} 2$ & Blank & -- & -- & -- & -- & -- & -- & -- & -- & -- & -- \\
\hline B3 & Blank & -- & -- & -- & -- & -- & -- & -- & -- & -- & -- \\
\hline B4 & Blank & -- & -- & -- & -- & -- & -- & -- & -- & -- & -- \\
\hline B5 & Blank & -- & -- & -- & -- & -- & -- & -- & -- & -- & -- \\
\hline B6 & Blank & -- & -- & -- & -- & -- & -- & -- & -- & -- & -- \\
\hline B7 & Blank & -- & -- & -- & -- & -- & -- & -- & -- & -- & -- \\
\hline B8 & Blank & -- & -- & -- & -- & -- & -- & -- & -- & -- & -- \\
\hline $\mathrm{C} 1$ & F3R010 & 5.991 & 3.475 & 0.461 & $0.132(\mathrm{Zr})$ & 1.656 & 6.260 & 3.631 & 0.482 & $0.138(\mathrm{Zr})$ & 1.730 \\
\hline $\mathrm{C} 2$ & R0R010 & 5.930 & 3.439 & 0.447 & -- & 1.693 & 6.196 & 3.594 & 0.467 & -- & 1.769 \\
\hline $\mathrm{C} 3$ & R3R010 & 5.886 & 3.414 & 0.443 & -- & 1.681 & 6.150 & 3.567 & 0.463 & -- & 1.757 \\
\hline $\mathrm{C} 4$ & D3R010 & 5.942 & 3.446 & 0.452 & $0.065(\mathrm{Ti})$ & 1.652 & 6.209 & 3.601 & 0.472 & $0.067(\mathrm{Ti})$ & 1.726 \\
\hline $\mathrm{C} 5$ & Blank & -- & -- & -- & -- & -- & -- & -- & -- & -- & -- \\
\hline C6 & Blank & -- & -- & -- & -- & -- & -- & -- & -- & -- & -- \\
\hline $\mathrm{C} 7$ & Blank & -- & -- & -- & -- & -- & -- & -- & -- & -- & -- \\
\hline $\mathrm{C} 8$ & Blank & -- & -- & -- & -- & -- & -- & -- & -- & -- & -- \\
\hline D1 & Blank & -- & -- & -- & -- & -- & -- & -- & -- & -- & -- \\
\hline $\mathrm{D} 2$ & Blank & -- & -- & -- & -- & -- & -- & -- & -- & -- & -- \\
\hline D3 & Blank & -- & -- & -- & -- & -- & -- & -- & -- & -- & -- \\
\hline D4 & Blank & -- & -- & -- & -- & -- & -- & -- & -- & -- & -- \\
\hline D5 & Blank & -- & -- & -- & -- & -- & -- & -- & -- & -- & -- \\
\hline D6 & Blank & -- & -- & -- & -- & -- & -- & -- & -- & -- & -- \\
\hline D7 & Blank & -- & -- & -- & -- & -- & -- & -- & -- & -- & -- \\
\hline D8 & Blank & -- & -- & -- & -- & -- & -- & -- & -- & -- & -- \\
\hline
\end{tabular}




\section{EXPERIMENT HARDWARE}

The experiment hardware configuration is identical for the RERTR-7A and -7B experiments. A list of irradiation hardware drawings used for analysis is given in Table 5.

Table 5. RERTR-7A/B irradiation hardware drawing list.

\begin{tabular}{cl}
\hline $\begin{array}{c}\text { Drawing } \\
\text { Number }\end{array}$ & \multicolumn{1}{c}{ Drawing Title } \\
\hline DWG-630223 & RERTR ATR Large B-Position Irradiation Experiment Assembly \\
DWG-630233 & ATR Large B-Position Basket \\
DWG-630231 & ATR Top Spacer Assembly \\
DWG-630225 & ATR Upper Spacer Assembly \\
DWG-630229 & ATR Bottom Spacer Assembly \\
DWG-630227 & ATR Large B-Position Fuel Capsule Assembly \\
DWG-630237 & Fuel Capsule \\
DWG-630239 & Capsule Cap \\
DWG-630244 & RERTR Mini-Plate \\
DWG-630238 & Fuel Plate, Dispersion \\
\hline
\end{tabular}

The RERTR miniplate irradiation assembly, (see Figure 1) shows the main components of the test assembly, which include the bottom spacer, upper and top spacers, experiment capsules and basket. The bottom spacer elevates the experiment capsules to the correct location in the core. The upper and top spacers allow the operators to assure that the experiment is seated fully into the basket. All spacers are similar to the capsule design except the spacers do not have the grooves for the plates. The capsules hold the fuel plates; a capsule cap is welded onto the top of the capsule to keep the plates from sliding out during handling and irradiation. The fuel plate drawings for monolithic and dispersion plates (DWG-630244 and DWG-630238, respectively) and RERTR miniplate capsule assembly are shown in Figure 2, Figure 3 and Figure 4, respectively. Each capsule has a notch at the top and a groove at the bottom which allow the capsules to stack and align properly into the core. The basket holds the test assembly in the reactor during irradiation, the notches on the outer wall allow for bypass coolant flow to cool the outer wall. The basket has two guide bars on the inside wall to guide the assembly into the baskets. 


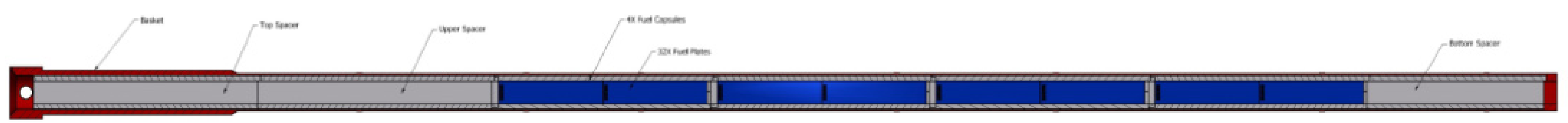

Figure 1 RERTR miniplate irradiation Assembly. 


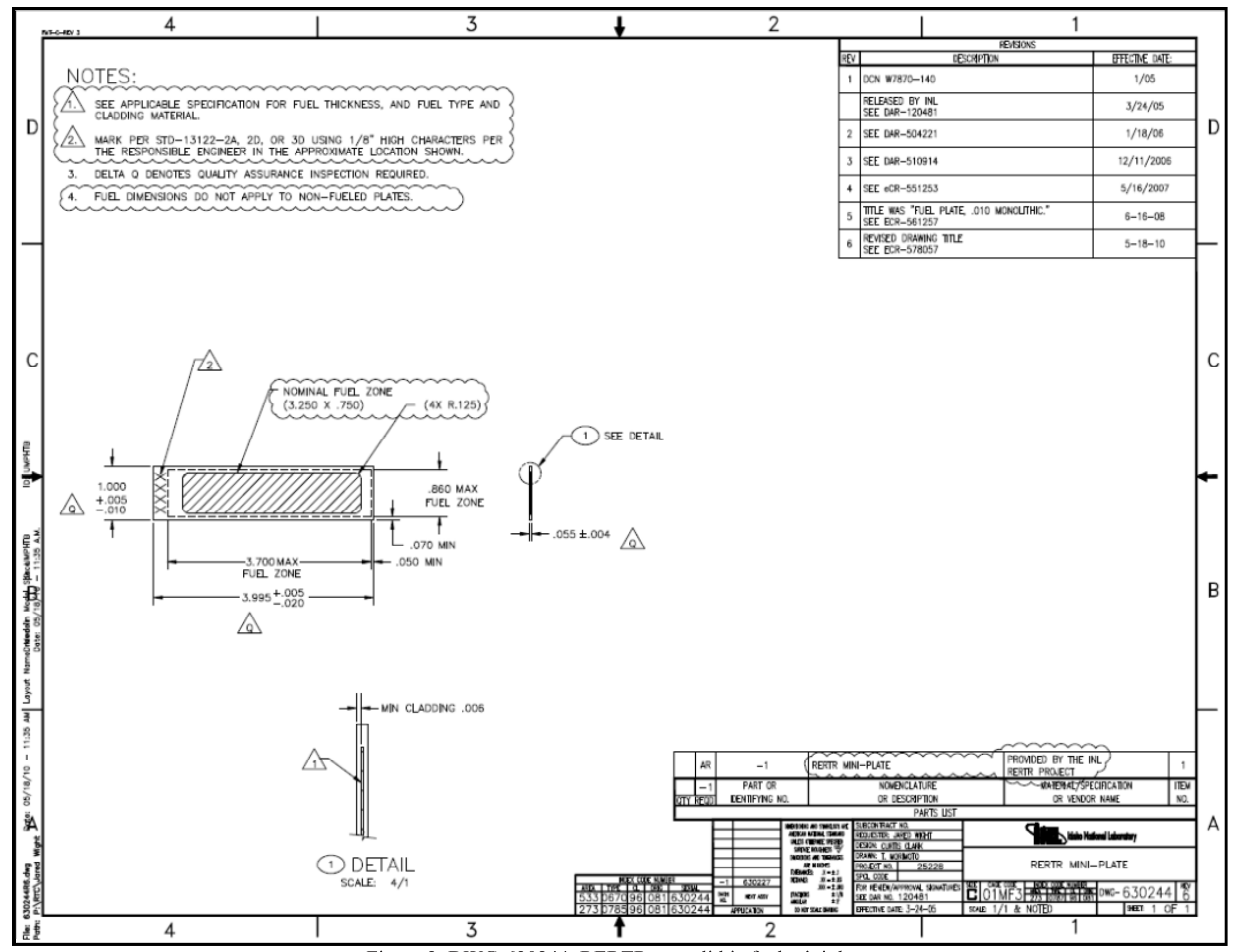

Figure 2. DWG-630244: RERTR monolithic fuel miniplate. 


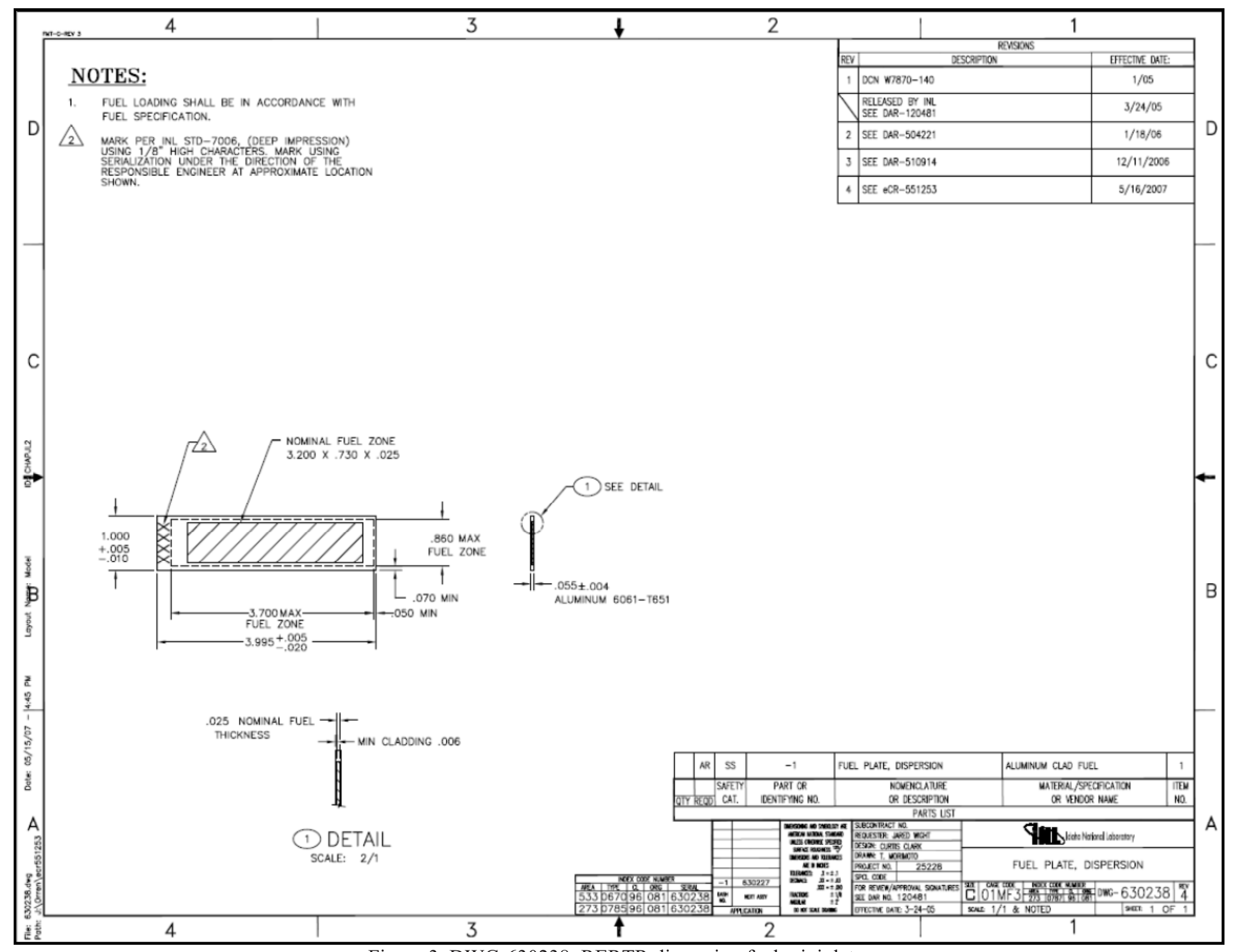

Figure 3. DWG-630238: RERTR dispersion fuel miniplate. 


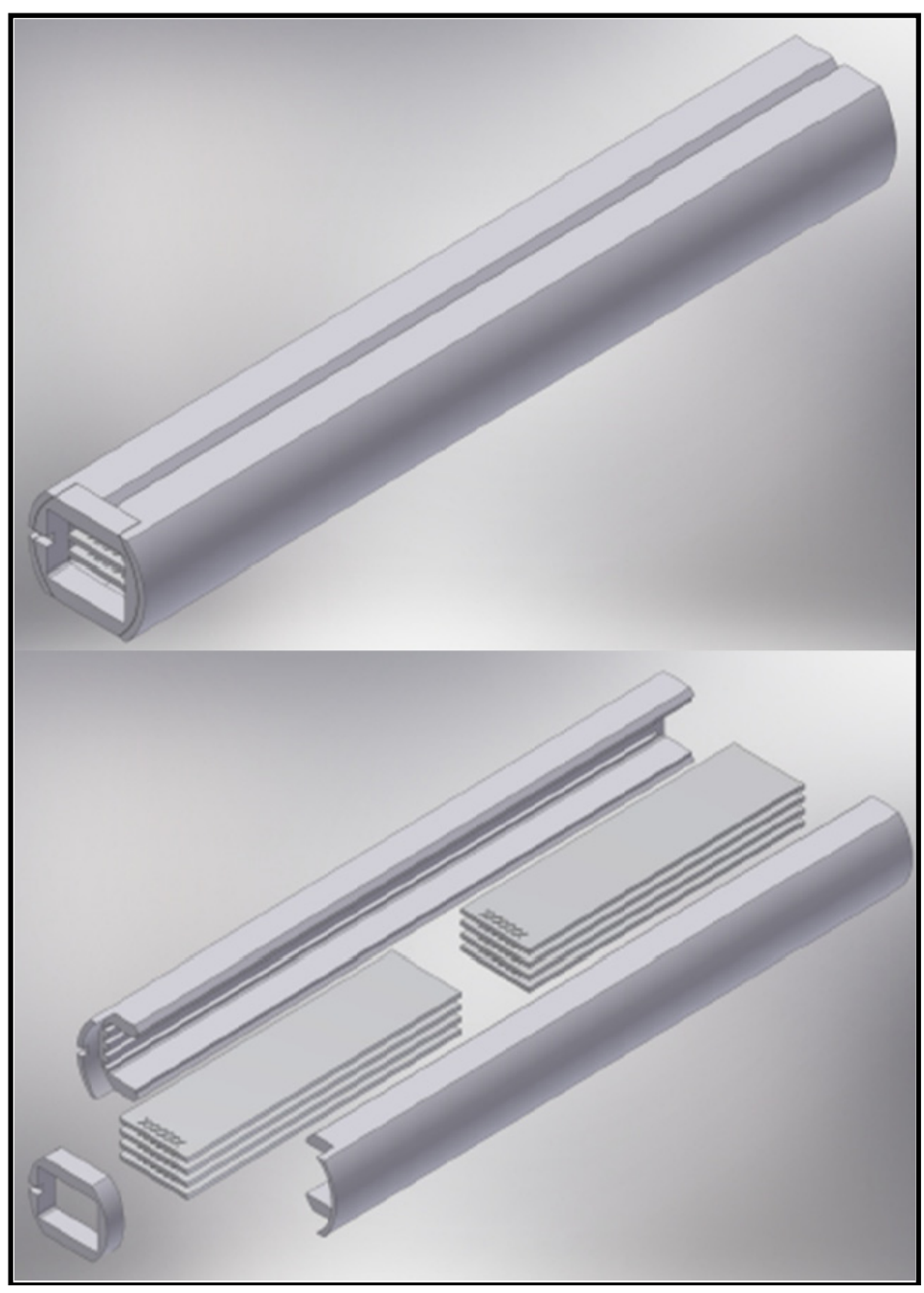

Figure 4. RERTR capsule assembly. 


\section{IRRADIATION HISTORY}

The RERTR-7A test assembly was irradiated in Cycles 136A and 136B in the large-B position B-11. The power of position B-11 in the core is represented by the south lobe power which is the average of the $\mathrm{SW}, \mathrm{C}$ and SE lobe powers, $\mathrm{S}=(\mathrm{SW}+\mathrm{C}+\mathrm{SE}) / 3$. Cycle 136A ran for 50.9 EFPDs with the south lobe power at 23.3 MW (total core power of $106 \mathrm{MW}$ ), Cycle 136B ran for 39 EFPDs with the south lobe power at 21.4 MW (total core power 105.9 MW). There was one mid-cycle SCRAM during cycle 136B with a duration of 7 days. This information is tabulated in Table 6.

The RERTR-7B test assembly was irradiated in Cycle 136B in the large-B position B-12. The power of position B-12 is represented by the west lobe power which is the average of the NW, SW and C lobe powers, $\mathrm{W}=(\mathrm{NW}+\mathrm{C}+\mathrm{SW}) / 3$. Cycle $136 \mathrm{~B}$ had a west lobe power of $21.6 \mathrm{MW}$; there was one midcycle SCRAM during cycle 136B with a duration of 7 days. This information is tabulated in Table 7.

Table 6. Irradiation history for the RERTR-7A experiment.

\begin{tabular}{|c|c|c|c|c|c|c|}
\hline ATR & $\begin{array}{c}\text { RERTR-7A } \\
\text { Capsules } \\
\text { Irradiated }\end{array}$ & Dates Irradiated & $\begin{array}{c}\text { Cycle } \\
\text { EFPDs }\end{array}$ & $\begin{array}{c}\text { Mid-Cycle } \\
\text { SCRAM } \\
\text { (days) }\end{array}$ & $\begin{array}{c}\text { South Lobe } \\
\text { Source } \\
\text { Power (MW) }\end{array}$ & $\begin{array}{c}\text { Total } \\
\text { Core } \\
\text { Power } \\
\text { (MW) }\end{array}$ \\
\hline 136A & A,B,C,D & $\begin{array}{c}11 / 24 / 2005- \\
1 / 14 / 2006\end{array}$ & 50.9 & 0 & 23.3 & 106.0 \\
\hline 136B & A,B,C,D & $\begin{array}{c}01 / 22 / 2006- \\
03 / 09 / 2006\end{array}$ & 39.0 & 7 & 23.3 & 105.9 \\
\hline
\end{tabular}

Table 7. Irradiation history for the RERTR-7B experiment.

\begin{tabular}{|c|c|c|c|c|c|c|}
\hline ATR & $\begin{array}{c}\text { RERTR-7B } \\
\text { Capsules } \\
\text { Irradiated }\end{array}$ & Dates Irradiated & $\begin{array}{c}\text { Cycle } \\
\text { EFPDs }\end{array}$ & $\begin{array}{c}\text { Mid-Cycle } \\
\text { SCRAM } \\
\text { (days) }\end{array}$ & $\begin{array}{c}\text { West Lobe } \\
\text { Source } \\
\text { Power } \\
\text { (MW) }\end{array}$ & $\begin{array}{c}\text { Total } \\
\text { Core } \\
\text { Power } \\
\text { (MW) }\end{array}$ \\
\hline 136B & $\mathrm{C}$ & $\begin{array}{c}01 / 22 / 2006- \\
03 / 09 / 2006\end{array}$ & 39.0 & 7 & 21.6 & 105.9 \\
\hline
\end{tabular}

The power history for each cycle is obtained as in ATR Surveillance Report from the ATR Data Acquisition System (DAS). The plot of each lobe power on an hourly basis for cycle 136B is shown in Figure 5.

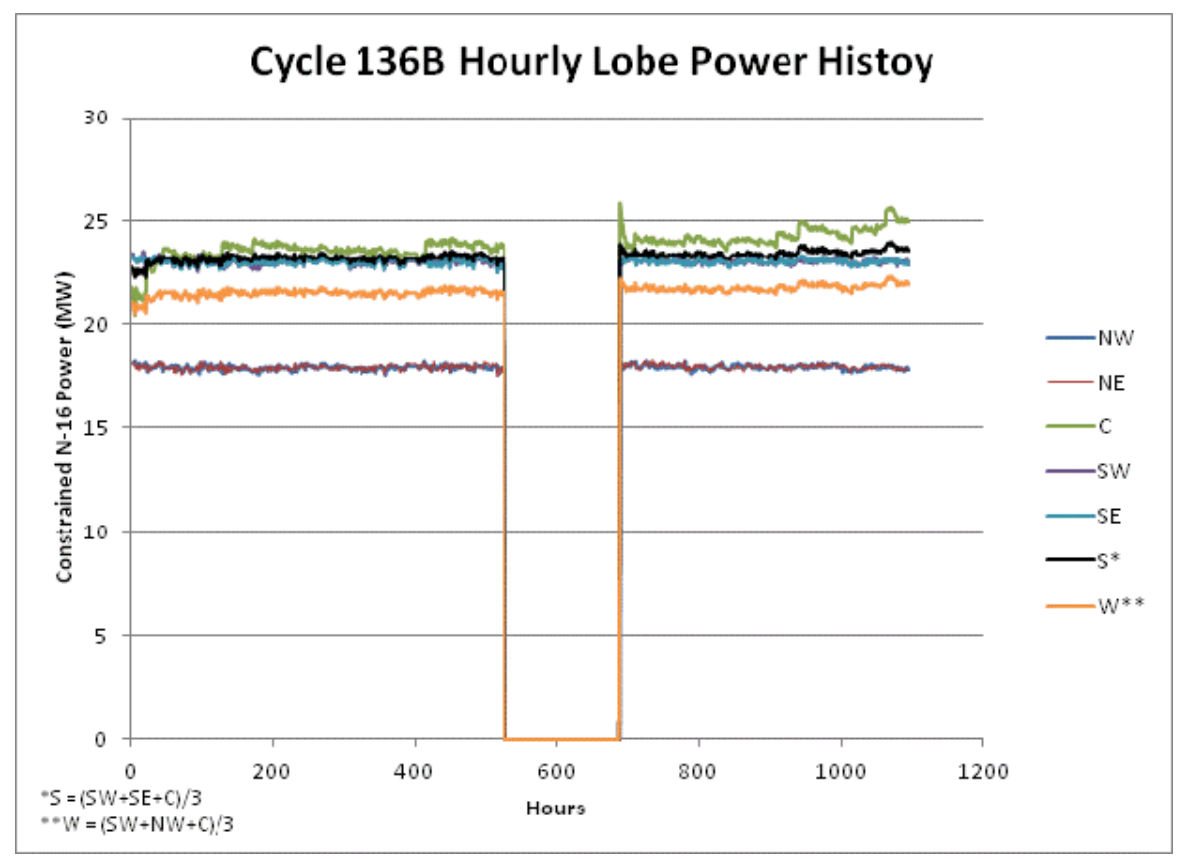

Figure 5. Hourly lobe power history for ATR Cycle 136B. 


\section{AS-RUN NUCLEAR ANALYSIS}

\subsection{Neutronics}

The as-run calculations were performed using the irradiation history in Table 6 and Table 7 and the Monte Carlo N-Particle (MCNP) code. The calculated as-run fission heat rates and as-run U-235 burnup results for the fueled miniplates reported have an uncertainty band $(1 \sigma)$ of $2.5 \%{ }^{4}$ The time intervals used to calculate average plate power and burnup for the plates in RERTR-7A and RERTR-7B are shown in Table 8 and Table 9, respectively. The RERTR-7A end of cycle average plate power and burnup for cycles 136A and 136B are shown in Table 12 and Table 15, respectively. The RERTR-7A average plate power and burnup for cycle 136A are shown in Table 10 through Table 12 and the RERTR-7A average plate power and burnup for cycle 136B are shown in Table 13 through Table 15. The RERTR-7B end of cycle average plate power and burnup for cycle 136B is shown in Table 18, with the average plate power and burnup for the entire cycle shown in Table 16 through Table 18. The plots of the power and burnup as a function of the ATR Cycle time interval are in Appendix A.

Table 8. RERTR-7A Cycle breakdown.

\begin{tabular}{ccc}
$\begin{array}{c}\text { Time } \\
\text { Interval }\end{array}$ & $\begin{array}{c}136 \mathrm{~A} \\
\text { (days) }\end{array}$ & $\begin{array}{c}136 \mathrm{~B} \\
\text { (days) }\end{array}$ \\
\hline 01 & $1.00 \mathrm{E}-4$ & $1.00 \mathrm{E}-4$ \\
02 & 15 & 12 \\
03 & 20 & 13.5 \\
04 & 15.9 & 13.5 \\
05 & $1.00 \mathrm{E}-3$ & $1.00 \mathrm{E}-3$ \\
EFPDs & 50.9 & 39 \\
\hline \hline Cumulative & 50.9 & 89.9 \\
\hline
\end{tabular}

Table 9. RERTR-7B Cycle breakdown.

\begin{tabular}{cc}
$\begin{array}{c}\text { Time } \\
\text { Interval }\end{array}$ & $\begin{array}{c}136 \mathrm{~B} \\
\text { (days) }\end{array}$ \\
\hline 01 & $1.00 \mathrm{E}-4$ \\
02 & 15 \\
03 & 15 \\
04 & 9 \\
05 & $1.00 \mathrm{E}-3$ \\
EFPDs & 39 \\
\hline \hline Cumulative & 39 \\
\hline
\end{tabular}


Table 10. RERTR-7A average plate power and burnup for MOC1 136A (15 EFPD).

\begin{tabular}{|c|c|c|c|c|c|c|}
\hline $\begin{array}{c}\text { Plate } \\
\text { Location }\end{array}$ & Plate ID & $\begin{array}{c}\text { Fission Heat } \\
\text { Rate } \\
(\mathrm{W} / \mathrm{g}) \\
\end{array}$ & $\begin{array}{c}\text { Fission Power } \\
\text { Density } \\
(\mathrm{W} / \mathrm{cc}) \\
\end{array}$ & $\begin{array}{c}\text { Surface Heat } \\
\text { Flux* } \\
\left(\mathrm{W} / \mathrm{cm}^{2}\right) \\
\end{array}$ & $\begin{array}{c}\text { U-235 } \\
\text { Burnup } \\
(\%) \\
\end{array}$ & $\begin{array}{c}\text { Fission } \\
\text { Density } \\
\text { (fissions/cc) } \\
\end{array}$ \\
\hline A1 & Blank & --- & ב-- & ב-- & -- & -- \\
\hline $\mathrm{A} 2$ & Blank & -- & -- & -- & -- & -- \\
\hline A3 & Blank & -- & -- & -- & -- & -- \\
\hline A4 & Blank & -- & -- & -- & -- & -- \\
\hline A5 & R3R040 & 984.22 & 8179.59 & 259.70 & $4.52 \%$ & $3.67 \mathrm{E}+20$ \\
\hline A6 & V5R040 & 793.59 & 6785.25 & 215.43 & $3.81 \%$ & $3.05 E+20$ \\
\hline A7 & R5R030 & 823.42 & 6850.52 & 217.50 & $3.81 \%$ & $3.08 \mathrm{E}+20$ \\
\hline A8 & H1F020 & 1168.61 & 18920.73 & 240.29 & $4.61 \%$ & $8.49 \mathrm{E}+20$ \\
\hline $\mathrm{B} 1$ & R1R040 & 1106.59 & 9209.71 & 292.41 & $5.07 \%$ & $4.14 \mathrm{E}+20$ \\
\hline $\mathrm{B} 2$ & R2R040 & 917.46 & 7624.66 & 242.08 & $4.26 \%$ & $3.42 \mathrm{E}+20$ \\
\hline B3 & R0R010 & 921.96 & 7660.44 & 243.22 & $4.26 \%$ & $3.44 \mathrm{E}+20$ \\
\hline B4 & H1T010 & 1342.34 & 21194.23 & 269.17 & $5.31 \%$ & $9.52 \mathrm{E}+20$ \\
\hline B5 & L1F01L & 1454.36 & 21604.47 & 274.38 & $5.49 \%$ & $9.70 \mathrm{E}+20$ \\
\hline B6 & V5R050 & 976.05 & 8314.10 & 263.97 & $4.57 \%$ & $3.73 \mathrm{E}+20$ \\
\hline B7 & L1F140 & 1263.18 & 20742.40 & 263.43 & $4.91 \%$ & $9.31 \mathrm{E}+20$ \\
\hline $\mathrm{B} 8$ & MZ 25 & 673.77 & 10773.19 & 136.82 & $7.26 \%$ & $4.84 \mathrm{E}+20$ \\
\hline $\mathrm{C} 1$ & H1F030 & 1359.10 & 22658.06 & 287.76 & $5.41 \%$ & $1.02 \mathrm{E}+21$ \\
\hline $\mathrm{C} 2$ & L1T020 & 1206.15 & 19408.08 & 246.48 & $4.62 \%$ & $8.71 \mathrm{E}+20$ \\
\hline $\mathrm{C} 3$ & L1F110 & 1227.84 & 20315.86 & 258.01 & $4.78 \%$ & $9.12 \mathrm{E}+20$ \\
\hline $\mathrm{C} 4$ & MZ 50 & 566.91 & 8493.51 & 215.74 & $6.31 \%$ & $3.81 \mathrm{E}+20$ \\
\hline $\mathrm{C} 5$ & L1F120 & 1364.67 & 22750.59 & 288.93 & $5.31 \%$ & $1.02 \mathrm{E}+21$ \\
\hline C6 & H1T020 & 1151.68 & 17833.67 & 226.49 & $4.57 \%$ & $8.01 \mathrm{E}+20$ \\
\hline $\mathrm{C} 7$ & R3R050 & 940.81 & 7836.72 & 248.82 & $4.31 \%$ & $3.52 \mathrm{E}+20$ \\
\hline $\mathrm{C} 8$ & R5R040 & 1131.48 & 9414.07 & 298.90 & $5.14 \%$ & $4.23 \mathrm{E}+20$ \\
\hline D1 & R1R050 & 1226.49 & 10155.30 & 322.43 & $5.59 \%$ & $4.56 \mathrm{E}+20$ \\
\hline D2 & Blank & -- & -- & -- & -- & -- \\
\hline D3 & R0R020 & 1226.64 & 10192.32 & 323.61 & $5.61 \%$ & $4.58 \mathrm{E}+20$ \\
\hline D4 & Blank & -- & -- & -- & -- & -- \\
\hline D5 & Blank & -- & -- & -- & -- & -- \\
\hline D6 & L1F160 & 1112.59 & 15155.51 & 192.47 & $4.33 \%$ & $6.80 \mathrm{E}+20$ \\
\hline D7 & L2F040 & 771.55 & 11696.54 & 297.09 & $3.05 \%$ & $5.25 \mathrm{E}+20$ \\
\hline D8 & R2R050 & 886.78 & 7369.40 & 233.98 & $4.07 \%$ & $3.31 \mathrm{E}+20$ \\
\hline
\end{tabular}

*Calculated using nominal fuel meat thickness $(0.0635 \mathrm{~cm}$ for dispersion, $0.0254 \mathrm{~cm}$ for thin monolithic and $0.0508 \mathrm{~cm}$ for thick monolithic). 
Table 11. RERTR-7A average plate power and burnup for MOC2 136A (35 EFPD).

\begin{tabular}{|c|c|c|c|c|c|c|}
\hline $\begin{array}{c}\text { Plate } \\
\text { Location }\end{array}$ & Plate ID & $\begin{array}{c}\text { Fission Heat } \\
\text { Rate } \\
(\mathrm{W} / \mathrm{g})\end{array}$ & $\begin{array}{c}\text { Fission Power } \\
\text { Density } \\
(\mathrm{W} / \mathrm{cc})\end{array}$ & $\begin{array}{c}\text { Surface Heat } \\
\text { Flux* } \\
\left(\mathrm{W} / \mathrm{cm}^{2}\right)\end{array}$ & $\begin{array}{c}\text { U-235 } \\
\text { Burnup } \\
(\%)\end{array}$ & $\begin{array}{c}\text { Fission } \\
\text { Density } \\
\text { (fissions/cc) }\end{array}$ \\
\hline A1 & Blank & -- & --- & --- & -- & -- \\
\hline $\mathrm{A} 2$ & Blank & -- & -- & -- & -- & -- \\
\hline A3 & Blank & -- & -- & -- & -- & -- \\
\hline A4 & Blank & -- & -- & -- & -- & -- \\
\hline A5 & R3R040 & 945.99 & 7740.44 & 245.76 & $10.19 \%$ & $8.31 \mathrm{E}+20$ \\
\hline A6 & V5R040 & 761.89 & 6433.03 & 204.25 & $8.50 \%$ & $6.90 \mathrm{E}+20$ \\
\hline A7 & R5R030 & 792.36 & 6507.73 & 206.62 & $8.58 \%$ & $6.97 \mathrm{E}+20$ \\
\hline A8 & H1F020 & 1128.09 & 17932.31 & 227.74 & $10.38 \%$ & $1.92 \mathrm{E}+21$ \\
\hline B1 & R1R040 & 1064.65 & 8706.95 & 276.45 & $11.35 \%$ & $9.35 \mathrm{E}+20$ \\
\hline $\mathrm{B} 2$ & R2R040 & 883.21 & 7235.05 & 229.71 & $9.55 \%$ & $7.75 \mathrm{E}+20$ \\
\hline B3 & R0R010 & 886.93 & 7263.98 & 230.63 & $9.61 \%$ & $7.79 \mathrm{E}+20$ \\
\hline B4 & H1T010 & 1291.95 & 19975.90 & 253.69 & $11.93 \%$ & $2.15 \mathrm{E}+21$ \\
\hline B5 & L1F01L & 1402.70 & 20639.31 & 262.12 & $12.36 \%$ & $2.19 \mathrm{E}+21$ \\
\hline B6 & V5R050 & 938.53 & 7872.84 & 249.96 & $10.35 \%$ & $8.45 \mathrm{E}+20$ \\
\hline B7 & L1F140 & 1216.15 & 19577.62 & 248.64 & $11.05 \%$ & $2.10 \mathrm{E}+21$ \\
\hline B8 & MZ 25 & 635.92 & 10061.71 & 127.78 & $16.19 \%$ & $1.09 \mathrm{E}+21$ \\
\hline C1 & H1F030 & 1309.49 & 21367.53 & 271.37 & $12.15 \%$ & $2.30 \mathrm{E}+21$ \\
\hline $\mathrm{C} 2$ & L1T020 & 1164.29 & 18381.64 & 233.45 & $10.38 \%$ & $1.97 \mathrm{E}+21$ \\
\hline $\mathrm{C} 3$ & L1F110 & 1183.49 & 19208.35 & 243.95 & $10.77 \%$ & $2.06 \mathrm{E}+21$ \\
\hline $\mathrm{C} 4$ & MZ 50 & 536.08 & 7960.10 & 202.19 & $14.01 \%$ & $8.58 \mathrm{E}+20$ \\
\hline $\mathrm{C} 5$ & L1F120 & 1314.00 & 21441.32 & 272.30 & $11.88 \%$ & $2.30 \mathrm{E}+21$ \\
\hline C6 & H1T020 & 1111.03 & 16897.81 & 214.60 & $10.20 \%$ & $1.81 \mathrm{E}+21$ \\
\hline $\mathrm{C} 7$ & R3R050 & 905.50 & 7433.72 & 236.02 & $9.78 \%$ & $7.97 \mathrm{E}+20$ \\
\hline $\mathrm{C} 8$ & R5R040 & 1086.78 & 8883.39 & 282.05 & $11.63 \%$ & $9.54 \mathrm{E}+20$ \\
\hline D1 & R1R050 & 1178.95 & 9574.09 & 303.98 & $12.45 \%$ & $1.03 \mathrm{E}+21$ \\
\hline D2 & Blank & -- & -- & -- & -- & -- \\
\hline D3 & R0R020 & 1179.74 & 9615.34 & 305.29 & $12.54 \%$ & $1.03 \mathrm{E}+21$ \\
\hline D4 & Blank & -- & -- & -- & -- & -- \\
\hline D5 & Blank & -- & -- & -- & -- & -- \\
\hline D6 & L1F160 & 1071.66 & 14346.93 & 182.21 & $9.75 \%$ & $1.54 \mathrm{E}+21$ \\
\hline D7 & L2F040 & 743.31 & 11132.24 & 282.76 & $6.93 \%$ & $1.19 \mathrm{E}+21$ \\
\hline D8 & R2R050 & 857.07 & 7024.94 & 223.04 & $9.23 \%$ & $7.51 \mathrm{E}+20$ \\
\hline
\end{tabular}

*Calculated using nominal fuel meat thickness $(0.0635 \mathrm{~cm}$ for dispersion, $0.0254 \mathrm{~cm}$ for thin monolithic and $0.0508 \mathrm{~cm}$ for thick monolithic). 
Table 12. RERTR-7A average plate power and burnup for EOC 136A (50.9 EFPD).

\begin{tabular}{|c|c|c|c|c|c|c|}
\hline $\begin{array}{c}\text { Plate } \\
\text { Location }\end{array}$ & Plate ID & $\begin{array}{c}\text { Fission Heat } \\
\text { Rate } \\
(\mathrm{W} / \mathrm{g}) \\
\end{array}$ & $\begin{array}{c}\text { Fission Power } \\
\text { Density } \\
(\mathrm{W} / \mathrm{cc}) \\
\end{array}$ & $\begin{array}{c}\text { Surface Heat } \\
\text { Flux* } \\
\left(\mathrm{W} / \mathrm{cm}^{2}\right) \\
\end{array}$ & $\begin{array}{c}\text { U-235 } \\
\text { Burnup } \\
(\%)\end{array}$ & $\begin{array}{c}\text { Fission } \\
\text { Density } \\
\text { (fissions/cc) } \\
\end{array}$ \\
\hline A1 & Blank & -- & ב-- & ב-- & -- & ב-- \\
\hline $\mathrm{A} 2$ & Blank & -- & -- & -- & -- & -- \\
\hline A3 & Blank & -- & -- & -- & -- & -- \\
\hline A4 & Blank & -- & -- & -- & -- & -- \\
\hline A5 & R3R040 & 936.35 & 7511.43 & 238.49 & $14.58 \%$ & $1.19 \mathrm{E}+21$ \\
\hline A6 & V5R040 & 762.93 & 6340.55 & 201.31 & $12.23 \%$ & $9.91 \mathrm{E}+20$ \\
\hline A7 & R5R030 & 790.67 & 6387.45 & 202.80 & $12.32 \%$ & $1.00 \mathrm{E}+21$ \\
\hline A8 & H1F020 & 1118.04 & 17357.77 & 220.44 & $14.80 \%$ & $2.75 \mathrm{E}+21$ \\
\hline $\mathrm{B} 1$ & R1R040 & 1051.19 & 8410.79 & 267.04 & $16.21 \%$ & $1.33 \mathrm{E}+21$ \\
\hline $\mathrm{B} 2$ & R2R040 & 882.71 & 7099.87 & 225.42 & $13.70 \%$ & $1.11 \mathrm{E}+21$ \\
\hline B3 & R0R010 & 886.80 & 7129.26 & 226.35 & $13.75 \%$ & $1.12 \mathrm{E}+21$ \\
\hline B4 & H1T010 & 1280.75 & 19275.79 & 244.80 & $17.04 \%$ & $3.06 \mathrm{E}+21$ \\
\hline B5 & L1F01L & 1388.89 & 19590.09 & 248.79 & $17.61 \%$ & $3.12 \mathrm{E}+21$ \\
\hline B6 & V5R050 & 937.40 & 7710.28 & 244.80 & $14.80 \%$ & $1.21 \mathrm{E}+21$ \\
\hline B7 & L1F140 & 1215.57 & 19080.13 & 242.32 & $15.86 \%$ & $3.01 \mathrm{E}+21$ \\
\hline B8 & MZ 25 & 604.96 & 9447.96 & 119.99 & $22.85 \%$ & $1.54 \mathrm{E}+21$ \\
\hline $\mathrm{C} 1$ & H1F030 & 1296.80 & 20595.75 & 261.57 & $17.33 \%$ & $3.28 \mathrm{E}+21$ \\
\hline $\mathrm{C} 2$ & L1T020 & 1165.30 & 17956.09 & 228.04 & $14.86 \%$ & $2.83 \mathrm{E}+21$ \\
\hline $\mathrm{C} 3$ & L1F110 & 1182.41 & 18720.59 & 237.75 & $15.41 \%$ & $2.95 \mathrm{E}+21$ \\
\hline $\mathrm{C} 4$ & MZ 50 & 517.16 & 7594.75 & 192.91 & $19.85 \%$ & $1.22 \mathrm{E}+21$ \\
\hline $\mathrm{C} 5$ & L1F120 & 1300.84 & 20654.37 & 262.31 & $16.93 \%$ & $3.29 \mathrm{E}+21$ \\
\hline C6 & H1T020 & 1112.02 & 16524.97 & 209.87 & $14.63 \%$ & $2.60 \mathrm{E}+21$ \\
\hline $\mathrm{C} 7$ & R3R050 & 904.16 & 7283.15 & 231.24 & $14.04 \%$ & $1.14 \mathrm{E}+21$ \\
\hline $\mathrm{C} 8$ & R5R040 & 1071.19 & 8559.10 & 271.75 & $16.58 \%$ & $1.36 \mathrm{E}+21$ \\
\hline D1 & R1R050 & 1160.17 & 9197.33 & 292.02 & $17.79 \%$ & $1.47 \mathrm{E}+21$ \\
\hline D2 & Blank & -- & -- & -- & -- & -- \\
\hline D3 & R0R020 & 1162.41 & 9246.84 & 293.59 & $17.90 \%$ & $1.47 \mathrm{E}+21$ \\
\hline D4 & Blank & -- & -- & -- & -- & -- \\
\hline D5 & Blank & -- & -- & -- & -- & -- \\
\hline D6 & L1F160 & 1063.10 & 13915.43 & 176.73 & $13.92 \%$ & $2.20 \mathrm{E}+21$ \\
\hline D7 & L2F040 & 746.57 & 11007.47 & 279.59 & $9.92 \%$ & $1.72 \mathrm{E}+21$ \\
\hline D8 & R2R050 & 884.53 & 6798.83 & 215.86 & $13.18 \%$ & $1.07 \mathrm{E}+21$ \\
\hline
\end{tabular}

*Calculated using nominal fuel meat thickness $(0.0635 \mathrm{~cm}$ for dispersion, $0.0254 \mathrm{~cm}$ for thin monolithic and $0.0508 \mathrm{~cm}$ for thick monolithic). 
Table 13. RERTR-7A average plate power and burnup for MOC1 136B (12.0 EFPD, 62.9 EFPD Cumulative).

\begin{tabular}{|c|c|c|c|c|c|c|}
\hline $\begin{array}{c}\text { Plate } \\
\text { Location }\end{array}$ & Plate ID & $\begin{array}{c}\text { Fission Heat } \\
\text { Rate } \\
(\mathrm{W} / \mathrm{g}) \\
\end{array}$ & $\begin{array}{c}\text { Fission Power } \\
\text { Density } \\
(\mathrm{W} / \mathrm{cc}) \\
\end{array}$ & $\begin{array}{c}\text { Surface Heat } \\
\text { Flux* } \\
\left(\mathrm{W} / \mathrm{cm}^{2}\right) \\
\end{array}$ & $\begin{array}{c}\text { U-235 } \\
\text { Burnup } \\
(\%) \\
\end{array}$ & $\begin{array}{c}\text { Fission } \\
\text { Density } \\
\text { (fissions/cc) } \\
\end{array}$ \\
\hline A1 & Blank & -- & --- & -- & -- & -- \\
\hline $\mathrm{A} 2$ & Blank & -- & -- & -- & -- & -- \\
\hline A3 & Blank & -- & -- & -- & -- & -- \\
\hline A4 & Blank & -- & -- & -- & -- & -- \\
\hline A5 & R3R040 & 927.90 & 7328.32 & 232.67 & $17.83 \%$ & $1.45 \mathrm{E}+21$ \\
\hline A6 & V5R040 & 763.82 & 6267.09 & 198.98 & $14.95 \%$ & $1.22 \mathrm{E}+21$ \\
\hline A7 & R5R030 & 793.14 & 6323.44 & 200.77 & $15.12 \%$ & $1.23 \mathrm{E}+21$ \\
\hline $\mathrm{A} 8$ & H1F020 & 1111.72 & 16942.59 & 215.17 & $18.06 \%$ & $3.36 \mathrm{E}+21$ \\
\hline $\mathrm{B} 1$ & R1R040 & 1043.94 & 8205.64 & 260.53 & $19.83 \%$ & $1.63 \mathrm{E}+21$ \\
\hline $\mathrm{B} 2$ & R2R040 & 885.50 & 7017.29 & 222.80 & $16.82 \%$ & $1.37 \mathrm{E}+21$ \\
\hline B3 & R0R010 & 888.12 & 7034.46 & 223.34 & $16.88 \%$ & $1.37 \mathrm{E}+21$ \\
\hline B4 & H1T010 & 1268.13 & 18680.23 & 237.24 & $20.81 \%$ & $3.74 \mathrm{E}+21$ \\
\hline B5 & L1F01L & 1379.79 & 19011.75 & 241.45 & $21.48 \%$ & $3.80 \mathrm{E}+21$ \\
\hline B6 & V5R050 & 941.31 & 7620.99 & 241.97 & $18.17 \%$ & $1.48 \mathrm{E}+21$ \\
\hline B7 & L1F140 & 1216.67 & 18707.40 & 237.58 & $19.36 \%$ & $3.68 \mathrm{E}+21$ \\
\hline $\mathrm{B} 8$ & MZ 25 & 582.67 & 9009.56 & 114.42 & $27.62 \%$ & $1.86 \mathrm{E}+21$ \\
\hline $\mathrm{C} 1$ & H1F030 & 1286.68 & 19991.53 & 253.89 & $21.09 \%$ & $3.99 \mathrm{E}+21$ \\
\hline $\mathrm{C} 2$ & L1T020 & 1171.72 & 17706.16 & 224.87 & $18.21 \%$ & $3.46 \mathrm{E}+21$ \\
\hline $\mathrm{C} 3$ & L1F110 & 1186.63 & 18421.44 & 233.95 & $18.88 \%$ & $3.61 \mathrm{E}+21$ \\
\hline $\mathrm{C} 4$ & MZ 50 & 503.72 & 7334.79 & 186.30 & $24.10 \%$ & $1.48 \mathrm{E}+21$ \\
\hline $\mathrm{C} 5$ & L1F120 & 1292.77 & 20084.50 & 255.07 & $20.68 \%$ & $4.01 \mathrm{E}+21$ \\
\hline C6 & H1T020 & 1115.14 & 16265.15 & 206.57 & $17.93 \%$ & $3.18 \mathrm{E}+21$ \\
\hline $\mathrm{C} 7$ & R3R050 & 906.15 & 7188.12 & 228.22 & $17.22 \%$ & $1.40 \mathrm{E}+21$ \\
\hline $\mathrm{C} 8$ & R5R040 & 1063.26 & 8346.28 & 264.99 & $20.20 \%$ & $1.66 \mathrm{E}+21$ \\
\hline D1 & R1R050 & 1147.55 & 8920.48 & 283.23 & $21.66 \%$ & $1.79 \mathrm{E}+21$ \\
\hline $\mathrm{D} 2$ & Blank & -- & -- & -- & -- & -- \\
\hline D3 & R0R020 & 1150.33 & 8973.83 & 284.92 & $21.84 \%$ & $1.80 \mathrm{E}+21$ \\
\hline D4 & Blank & -- & -- & -- & -- & -- \\
\hline D5 & Blank & -- & -- & -- & -- & -- \\
\hline D6 & L1F160 & 1058.07 & 13606.48 & 172.80 & $17.04 \%$ & $2.69 \mathrm{E}+21$ \\
\hline D7 & L2F040 & 570.82 & 10932.76 & 277.69 & $12.18 \%$ & $2.11 \mathrm{E}+21$ \\
\hline D8 & R2R050 & 839.18 & 6662.31 & 211.53 & $16.17 \%$ & $1.31 \mathrm{E}+21$ \\
\hline
\end{tabular}

*Calculated using nominal fuel meat thickness $(0.0635 \mathrm{~cm}$ for dispersion, $0.0254 \mathrm{~cm}$ for thin monolithic and $0.0508 \mathrm{~cm}$ for thick monolithic). 
Table 14. RERTR-7A average plate power and burnup for MOC2 136B (25.5 EFPD, 76.4 EFPD Cumulative).

\begin{tabular}{|c|c|c|c|c|c|c|}
\hline $\begin{array}{c}\text { Plate } \\
\text { Location }\end{array}$ & Plate ID & $\begin{array}{c}\text { Fission Heat } \\
\text { Rate } \\
(\mathrm{W} / \mathrm{g}) \\
\end{array}$ & $\begin{array}{c}\text { Fission Power } \\
\text { Density } \\
(\mathrm{W} / \mathrm{cc}) \\
\end{array}$ & $\begin{array}{c}\text { Surface Heat } \\
\text { Flux* } \\
\left(\mathrm{W} / \mathrm{cm}^{2}\right) \\
\end{array}$ & $\begin{array}{c}\text { U-235 } \\
\text { Burnup } \\
(\%) \\
\end{array}$ & $\begin{array}{c}\text { Fission } \\
\text { Density } \\
\text { (fissions/cc) } \\
\end{array}$ \\
\hline A1 & Blank & -- & --- & -- & -- & -- \\
\hline $\mathrm{A} 2$ & Blank & -- & -- & -- & -- & -- \\
\hline A3 & Blank & -- & -- & -- & -- & -- \\
\hline A4 & Blank & -- & -- & -- & -- & -- \\
\hline $\mathrm{A} 5$ & R3R040 & 922.27 & 7195.90 & 228.47 & $21.33 \%$ & $1.74 \mathrm{E}+21$ \\
\hline A6 & V5R040 & 963.11 & 6201.90 & 196.91 & $18.05 \%$ & $1.47 \mathrm{E}+21$ \\
\hline A7 & R5R030 & 793.95 & 6266.50 & 198.96 & $18.23 \%$ & $1.48 \mathrm{E}+21$ \\
\hline $\mathrm{A} 8$ & H1F020 & 1103.84 & 16585.84 & 210.64 & $21.69 \%$ & $4.03 \mathrm{E}+21$ \\
\hline $\mathrm{B} 1$ & R1R040 & 1034.86 & 8027.02 & 254.86 & $23.75 \%$ & $1.95 \mathrm{E}+21$ \\
\hline $\mathrm{B} 2$ & R2R040 & 884.49 & 6930.56 & 220.05 & $20.25 \%$ & $1.65 \mathrm{E}+21$ \\
\hline B3 & R0R010 & 886.02 & 6938.92 & 220.31 & $20.32 \%$ & $1.65 \mathrm{E}+21$ \\
\hline B4 & H1T010 & 1255.18 & 18192.08 & 231.04 & $24.87 \%$ & $4.47 \mathrm{E}+21$ \\
\hline B5 & L1F01L & 1370.59 & 18555.87 & 235.66 & $25.70 \%$ & $4.55 \mathrm{E}+21$ \\
\hline B6 & V5R050 & 939.65 & 7518.59 & 238.72 & $21.91 \%$ & $1.79 \mathrm{E}+21$ \\
\hline B7 & L1F140 & 1213.60 & 18374.46 & 233.36 & $23.27 \%$ & $4.42 \mathrm{E}+21$ \\
\hline $\mathrm{B} 8$ & MZ 25 & 563.70 & 8651.81 & 109.88 & $32.71 \%$ & $2.21 \mathrm{E}+21$ \\
\hline $\mathrm{C} 1$ & H1F030 & 1274.95 & 19490.73 & 247.53 & $25.26 \%$ & $4.78 \mathrm{E}+21$ \\
\hline $\mathrm{C} 2$ & L1T020 & 1173.34 & 17466.45 & 221.82 & $21.93 \%$ & $4.17 \mathrm{E}+21$ \\
\hline $\mathrm{C} 3$ & L1F110 & 1187.80 & 18160.93 & 230.64 & $22.67 \%$ & $4.35 \mathrm{E}+21$ \\
\hline $\mathrm{C} 4$ & MZ 50 & 492.90 & 7131.69 & 181.14 & $28.70 \%$ & $1.77 \mathrm{E}+21$ \\
\hline $\mathrm{C} 5$ & L1F120 & 1282.95 & 19602.16 & 248.95 & $24.74 \%$ & $4.80 \mathrm{E}+21$ \\
\hline C6 & H1T020 & 1114.53 & 16028.10 & 203.56 & $21.62 \%$ & $3.83 \mathrm{E}+21$ \\
\hline $\mathrm{C} 7$ & R3R050 & 906.36 & 7107.72 & 225.67 & $20.71 \%$ & $1.69 \mathrm{E}+21$ \\
\hline $\mathrm{C} 8$ & R5R040 & 1054.77 & 8167.82 & 259.33 & $24.20 \%$ & $1.99 \mathrm{E}+21$ \\
\hline D1 & R1R050 & 1134.67 & 8694.53 & 276.05 & $25.92 \%$ & $2.14 \mathrm{E}+21$ \\
\hline $\mathrm{D} 2$ & Blank & -- & -- & -- & -- & -- \\
\hline D3 & R0R020 & 1135.60 & 8727.97 & 277.11 & $26.11 \%$ & $2.15 \mathrm{E}+21$ \\
\hline D4 & Blank & -- & -- & -- & -- & -- \\
\hline D5 & Blank & -- & -- & -- & -- & -- \\
\hline D6 & L1F160 & 1049.97 & 13318.72 & 169.15 & $20.49 \%$ & $3.23 \mathrm{E}+21$ \\
\hline D7 & L2F040 & 754.57 & 10881.35 & 276.39 & $14.73 \%$ & $2.55 \mathrm{E}+21$ \\
\hline D8 & R2R050 & 833.21 & 6542.98 & 207.74 & $19.43 \%$ & $1.58 \mathrm{E}+21$ \\
\hline
\end{tabular}

*Calculated using nominal fuel meat thickness $(0.0635 \mathrm{~cm}$ for dispersion, $0.0254 \mathrm{~cm}$ for thin monolithic and $0.0508 \mathrm{~cm}$ for thick monolithic). 
Table 15. RERTR-7A average plate power and burnup for EOC 136B (39 EFPD, 89.9 EFPD Cumulative).

\begin{tabular}{|c|c|c|c|c|c|c|}
\hline $\begin{array}{c}\text { Plate } \\
\text { Location }\end{array}$ & Plate ID & $\begin{array}{c}\text { Fission Heat } \\
\text { Rate } \\
(\mathrm{W} / \mathrm{g}) \\
\end{array}$ & $\begin{array}{c}\text { Fission Power } \\
\text { Density } \\
(\mathrm{W} / \mathrm{cc})\end{array}$ & $\begin{array}{c}\text { Surface Heat } \\
\text { Flux* } \\
\left(\mathrm{W} / \mathrm{cm}^{2}\right) \\
\end{array}$ & $\begin{array}{c}\text { U-235 } \\
\text { Burnup } \\
(\%)\end{array}$ & $\begin{array}{c}\text { Fission } \\
\text { Density } \\
\text { (fissions/cc) } \\
\end{array}$ \\
\hline A1 & Blank & (-- & --- & - & -- & -- \\
\hline $\mathrm{A} 2$ & Blank & -- & -- & -- & -- & -- \\
\hline A3 & Blank & -- & -- & -- & -- & -- \\
\hline A4 & Blank & -- & -- & -- & -- & -- \\
\hline A5 & R3R040 & 913.62 & 7037.27 & 223.43 & $24.83 \%$ & $2.03 \mathrm{E}+21$ \\
\hline A6 & V5R040 & 762.24 & 6125.01 & 194.47 & $21.03 \%$ & $1.71 \mathrm{E}+21$ \\
\hline A7 & R5R030 & 790.83 & 6169.56 & 195.88 & $21.28 \%$ & $1.73 \mathrm{E}+21$ \\
\hline A8 & H1F020 & 1095.89 & 16204.74 & 205.80 & $25.19 \%$ & $4.68 \mathrm{E}+21$ \\
\hline $\mathrm{B} 1$ & R1R040 & 1023.00 & 7817.30 & 248.20 & $27.55 \%$ & $2.27 \mathrm{E}+21$ \\
\hline $\mathrm{B} 2$ & R2R040 & 884.75 & 6843.85 & 217.29 & $23.63 \%$ & $1.92 \mathrm{E}+21$ \\
\hline B3 & R0R010 & 885.59 & 6848.70 & 217.45 & $23.69 \%$ & $1.93 \mathrm{E}+21$ \\
\hline B4 & H1T010 & 1240.90 & 17666.12 & 224.36 & $28.83 \%$ & $5.18 \mathrm{E}+21$ \\
\hline B5 & L1F01L & 1356.32 & 18004.52 & 228.66 & $29.83 \%$ & $5.28 \mathrm{E}+21$ \\
\hline B6 & V5R050 & 934.87 & 7379.88 & 234.31 & $25.54 \%$ & $2.09 \mathrm{E}+21$ \\
\hline B7 & L1F140 & 1211.13 & 18022.91 & 228.89 & $27.09 \%$ & $5.15 \mathrm{E}+21$ \\
\hline B8 & MZ 25 & 542.89 & 8266.35 & 104.98 & $37.52 \%$ & $2.54 \mathrm{E}+21$ \\
\hline $\mathrm{C} 1$ & H1F030 & 1261.36 & 18931.22 & 240.43 & $29.30 \%$ & $5.55 \mathrm{E}+21$ \\
\hline $\mathrm{C} 2$ & L1T020 & 1173.89 & 17182.25 & 218.21 & $25.56 \%$ & $4.86 \mathrm{E}+21$ \\
\hline $\mathrm{C} 3$ & L1F110 & 1184.06 & 17797.85 & 226.03 & $26.41 \%$ & $5.07 \mathrm{E}+21$ \\
\hline $\mathrm{C} 4$ & MZ 50 & 479.45 & 6888.12 & 174.96 & $33.11 \%$ & $2.05 \mathrm{E}+21$ \\
\hline $\mathrm{C} 5$ & L1F120 & 1270.01 & 19051.21 & 241.95 & $28.67 \%$ & $5.57 \mathrm{E}+21$ \\
\hline C6 & H1T020 & 1115.35 & 15781.65 & 200.43 & $25.17 \%$ & $4.47 \mathrm{E}+21$ \\
\hline $\mathrm{C} 7$ & R3R050 & 904.55 & 7001.17 & 222.29 & $24.20 \%$ & $1.97 \mathrm{E}+21$ \\
\hline $\mathrm{C} 8$ & R5R040 & 1043.69 & 7962.17 & 252.80 & $28.15 \%$ & $2.31 \mathrm{E}+21$ \\
\hline D1 & R1R050 & 1118.95 & 8434.68 & 267.80 & $30.05 \%$ & $2.48 \mathrm{E}+21$ \\
\hline D2 & Blank & -- & -- & -- & -- & -- \\
\hline D3 & R0R020 & 1121.57 & 8483.28 & 269.34 & $30.25 \%$ & $2.49 \mathrm{E}+21$ \\
\hline D4 & Blank & -- & -- & -- & -- & -- \\
\hline D5 & Blank & -- & -- & -- & -- & -- \\
\hline D6 & L1F160 & 1042.32 & 13024.55 & 165.41 & $23.78 \%$ & $3.75 \mathrm{E}+21$ \\
\hline D7 & L2F040 & 759.86 & 10842.23 & 275.39 & $17.24 \%$ & $2.99 \mathrm{E}+21$ \\
\hline D8 & R2R050 & 826.32 & 6410.15 & 203.52 & $22.61 \%$ & $1.84 \mathrm{E}+21$ \\
\hline
\end{tabular}

* Calculated using nominal fuel meat thickness $(0.0635 \mathrm{~cm}$ for dispersion, $0.0254 \mathrm{~cm}$ for thin monolithic and $0.0508 \mathrm{~cm}$ for thick monolithic). 
Table 16. RERTR-7B average plate power and burnup for MOC1 136B (15 EFPD).

\begin{tabular}{|c|c|c|c|c|c|c|}
\hline $\begin{array}{c}\text { Plate } \\
\text { Location }\end{array}$ & Plate ID & $\begin{array}{c}\text { Fission Heat } \\
\text { Rate } \\
(\mathrm{W} / \mathrm{g})\end{array}$ & $\begin{array}{c}\text { Fission Power } \\
\text { Density } \\
(\mathrm{W} / \mathrm{cc}) \\
\end{array}$ & $\begin{array}{c}\text { Surface Heat } \\
\text { Flux* } \\
\left(\mathrm{W} / \mathrm{cm}^{2}\right) \\
\end{array}$ & $\begin{array}{c}\text { U-235 } \\
\text { Burnup } \\
(\%) \\
\end{array}$ & $\begin{array}{c}\text { Fission } \\
\text { Density } \\
\text { (fissions/cc) } \\
\end{array}$ \\
\hline A1 & Blank & -- & -- & -- & -- & -- \\
\hline $\mathrm{A} 2$ & Blank & -- & -- & -- & -- & -- \\
\hline A3 & Blank & -- & -- & -- & -- & -- \\
\hline A4 & Blank & -- & -- & -- & -- & -- \\
\hline A5 & Blank & -- & -- & -- & -- & -- \\
\hline A6 & Blank & -- & -- & -- & -- & -- \\
\hline A7 & Blank & -- & -- & -- & -- & -- \\
\hline A8 & Blank & -- & -- & -- & -- & -- \\
\hline $\mathrm{B} 1$ & Blank & -- & -- & -- & -- & -- \\
\hline $\mathrm{B} 2$ & Blank & -- & -- & -- & -- & -- \\
\hline B3 & Blank & -- & -- & -- & -- & -- \\
\hline B4 & Blank & -- & -- & -- & -- & -- \\
\hline B5 & Blank & -- & -- & -- & -- & -- \\
\hline B6 & Blank & -- & -- & -- & -- & -- \\
\hline B7 & Blank & -- & -- & -- & -- & -- \\
\hline B8 & Blank & -- & -- & -- & -- & -- \\
\hline $\mathrm{C} 1$ & F3R010 & 868.39 & 7376.10 & 234.19 & $4.15 \%$ & $3.31 \mathrm{E}+20$ \\
\hline $\mathrm{C} 2$ & R0R010 & 717.49 & 6053.46 & 192.20 & $3.46 \%$ & $2.72 \mathrm{E}+20$ \\
\hline $\mathrm{C} 3$ & R3R010 & 729.68 & 6021.32 & 191.18 & $3.49 \%$ & $2.70 \mathrm{E}+20$ \\
\hline $\mathrm{C} 4$ & D3R010 & 881.96 & 7368.78 & 233.96 & $4.18 \%$ & $3.31 \mathrm{E}+20$ \\
\hline $\mathrm{C} 5$ & Blank & -- & -- & -- & -- & -- \\
\hline C6 & Blank & -- & -- & -- & -- & -- \\
\hline $\mathrm{C} 7$ & Blank & -- & -- & -- & -- & -- \\
\hline $\mathrm{C} 8$ & Blank & -- & -- & -- & -- & -- \\
\hline D1 & Blank & --- & -- & -- & -- & --- \\
\hline $\mathrm{D} 2$ & Blank & -- & -- & -- & -- & -- \\
\hline D3 & Blank & -- & -- & -- & -- & -- \\
\hline D4 & Blank & -- & -- & -- & -- & -- \\
\hline D5 & Blank & -- & -- & -- & -- & -- \\
\hline D6 & Blank & -- & -- & -- & -- & -- \\
\hline D7 & Blank & -- & -- & -- & -- & -- \\
\hline D8 & Blank & -- & -- & -- & -- & -- \\
\hline
\end{tabular}

*Calculated using nominal fuel meat thickness of $0.0635 \mathrm{~cm}$. 
Table 17. RERTR-7B average plate power and burnup for MOC2 136B (30 EFPD).

\begin{tabular}{|c|c|c|c|c|c|c|}
\hline $\begin{array}{c}\text { Plate } \\
\text { Location }\end{array}$ & Plate ID & $\begin{array}{c}\text { Fission Heat } \\
\text { Rate } \\
(\mathrm{W} / \mathrm{g}) \\
\end{array}$ & $\begin{array}{c}\text { Fission Power } \\
\text { Density } \\
(\mathrm{W} / \mathrm{cc}) \\
\end{array}$ & $\begin{array}{c}\text { Surface Heat } \\
\text { Flux* } \\
\left(\mathrm{W} / \mathrm{cm}^{2}\right) \\
\end{array}$ & $\begin{array}{c}\text { U-235 } \\
\text { Burnup } \\
(\%) \\
\end{array}$ & $\begin{array}{c}\text { Fission } \\
\text { Density } \\
\text { (fissions/cc) } \\
\end{array}$ \\
\hline A1 & Blank & +-- & +-- & +-- & --- & ב-- \\
\hline $\mathrm{A} 2$ & Blank & -- & -- & -- & -- & -- \\
\hline A3 & Blank & -- & -- & -- & -- & -- \\
\hline A4 & Blank & -- & -- & -- & -- & -- \\
\hline A5 & Blank & -- & -- & -- & -- & -- \\
\hline A6 & Blank & -- & -- & -- & -- & -- \\
\hline A7 & Blank & -- & -- & -- & -- & -- \\
\hline $\mathrm{A} 8$ & Blank & -- & -- & -- & -- & -- \\
\hline B1 & Blank & +-- & ב-- & +-- & -- & -- \\
\hline $\mathrm{B} 2$ & Blank & -- & -- & -- & -- & -- \\
\hline B3 & Blank & -- & -- & -- & -- & -- \\
\hline B4 & Blank & -- & -- & -- & -- & -- \\
\hline B5 & Blank & -- & -- & -- & -- & -- \\
\hline B6 & Blank & -- & -- & -- & -- & -- \\
\hline B7 & Blank & -- & -- & -- & -- & -- \\
\hline B8 & Blank & -- & -- & -- & -- & -- \\
\hline $\mathrm{C} 1$ & F3R010 & 835.49 & 6999.74 & 222.24 & $8.09 \%$ & $6.45 \mathrm{E}+20$ \\
\hline $\mathrm{C} 2$ & R0R010 & 689.63 & 5754.27 & 182.70 & $6.73 \%$ & $5.30 \mathrm{E}+20$ \\
\hline $\mathrm{C} 3$ & R3R010 & 701.33 & 5720.05 & 181.61 & $6.78 \%$ & $5.27 \mathrm{E}+20$ \\
\hline $\mathrm{C} 4$ & D3R010 & 849.24 & 6999.44 & 222.23 & $8.16 \%$ & $6.45 \mathrm{E}+20$ \\
\hline $\mathrm{C} 5$ & Blank & -- & -- & -- & -- & -- \\
\hline C6 & Blank & -- & -- & -- & -- & -- \\
\hline $\mathrm{C} 7$ & Blank & -- & -- & -- & -- & -- \\
\hline $\mathrm{C} 8$ & Blank & -- & -- & -- & -- & -- \\
\hline D1 & Blank & -- & -- & -- & -- & -- \\
\hline D2 & Blank & -- & -- & -- & -- & -- \\
\hline D3 & Blank & -- & -- & -- & -- & -- \\
\hline D4 & Blank & -- & -- & -- & -- & -- \\
\hline D5 & Blank & -- & -- & -- & -- & -- \\
\hline D6 & Blank & -- & -- & -- & -- & -- \\
\hline D7 & Blank & -- & -- & -- & -- & -- \\
\hline D8 & Blank & -- & -- & -- & -- & -- \\
\hline
\end{tabular}

*Calculated using nominal fuel meat thickness of $0.0635 \mathrm{~cm}$. 
Table 18. RERTR-7B average plate power and burnup for EOC 136B (39 EFPD).

\begin{tabular}{|c|c|c|c|c|c|c|}
\hline $\begin{array}{c}\text { Plate } \\
\text { Location }\end{array}$ & Plate ID & $\begin{array}{c}\text { Fission Heat } \\
\text { Rate } \\
(\mathrm{W} / \mathrm{g}) \\
\end{array}$ & $\begin{array}{c}\text { Fission Power } \\
\text { Density } \\
(\mathrm{W} / \mathrm{cc}) \\
\end{array}$ & $\begin{array}{c}\text { Surface Heat } \\
\text { Flux* } \\
\left(\mathrm{W} / \mathrm{cm}^{2}\right) \\
\end{array}$ & $\begin{array}{c}\text { U-235 } \\
\text { Burnup } \\
(\%) \\
\end{array}$ & $\begin{array}{c}\text { Fission } \\
\text { Density } \\
\text { (fissions/cc) } \\
\end{array}$ \\
\hline A1 & Blank & "-- & ב-- & ב-- & --- & ב-- \\
\hline $\mathrm{A} 2$ & Blank & -- & -- & -- & -- & -- \\
\hline A3 & Blank & -- & -- & -- & -- & -- \\
\hline A4 & Blank & -- & -- & -- & -- & -- \\
\hline A5 & Blank & -- & -- & -- & -- & -- \\
\hline A6 & Blank & -- & -- & -- & -- & -- \\
\hline A7 & Blank & -- & -- & -- & -- & -- \\
\hline A8 & Blank & -- & -- & -- & -- & -- \\
\hline $\mathrm{B} 1$ & Blank & ב-- & ב-- & ב-- & - & ב-- \\
\hline $\mathrm{B} 2$ & Blank & -- & -- & -- & -- & -- \\
\hline B3 & Blank & -- & -- & -- & -- & -- \\
\hline B4 & Blank & -- & -- & -- & -- & -- \\
\hline B5 & Blank & -- & -- & -- & -- & -- \\
\hline B6 & Blank & -- & -- & -- & -- & -- \\
\hline B7 & Blank & -- & -- & -- & -- & -- \\
\hline B8 & Blank & -- & -- & -- & -- & -- \\
\hline $\mathrm{C} 1$ & F3R010 & 827.94 & 6847.06 & 217.39 & $10.42 \%$ & $8.30 \mathrm{E}+20$ \\
\hline $\mathrm{C} 2$ & R0R010 & 689.19 & 5689.95 & 180.66 & $8.69 \%$ & $6.83 \mathrm{E}+20$ \\
\hline $\mathrm{C} 3$ & R3R010 & 700.30 & 5650.02 & 179.39 & $8.76 \%$ & $6.79 \mathrm{E}+20$ \\
\hline $\mathrm{C} 4$ & D3R010 & 842.81 & 6853.73 & 217.61 & $10.51 \%$ & $8.30 \mathrm{E}+20$ \\
\hline $\mathrm{C} 5$ & Blank & -- & -- & -- & -- & -- \\
\hline C6 & Blank & -- & -- & -- & -- & -- \\
\hline $\mathrm{C} 7$ & Blank & -- & -- & -- & -- & -- \\
\hline $\mathrm{C} 8$ & Blank & -- & -- & -- & -- & -- \\
\hline D1 & Blank & -- & -- & -- & -- & -- \\
\hline D2 & Blank & -- & -- & -- & -- & -- \\
\hline D3 & Blank & -- & -- & -- & -- & -- \\
\hline D4 & Blank & -- & -- & -- & -- & -- \\
\hline D5 & Blank & -- & -- & -- & -- & -- \\
\hline D6 & Blank & -- & -- & -- & -- & -- \\
\hline D7 & Blank & -- & -- & -- & -- & -- \\
\hline D8 & Blank & -- & -- & -- & -- & -- \\
\hline
\end{tabular}

*Calculated using nominal fuel meat thickness of $0.0635 \mathrm{~cm}$. 


\subsection{Gradients}

The MCNP-calculated power gradients ${ }^{6}$ in the transverse directions are represented by the thermal neutron flux and fission rate local-2-average ratios (L2ARs) as a function of position. Figure 6 and Figure 7 depict the power gradient in the transverse direction for the RERTR-7A experiment; the gradients were not calculated for the RERTR-7B experiment.

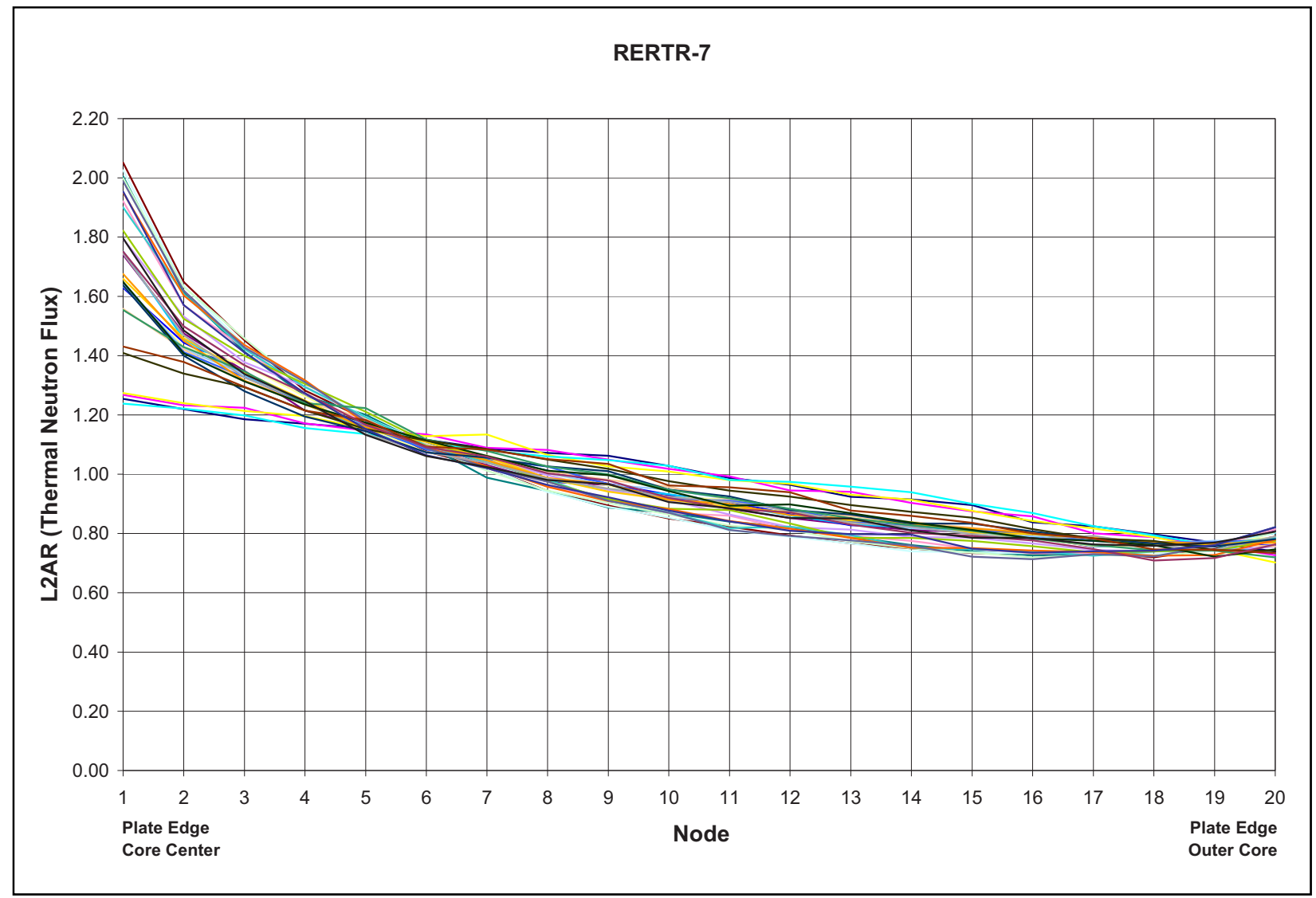

Figure 6. RERTR-7A fuel miniplates thermal neutron flux L2ARs in transverse direction. ${ }^{6}$ 


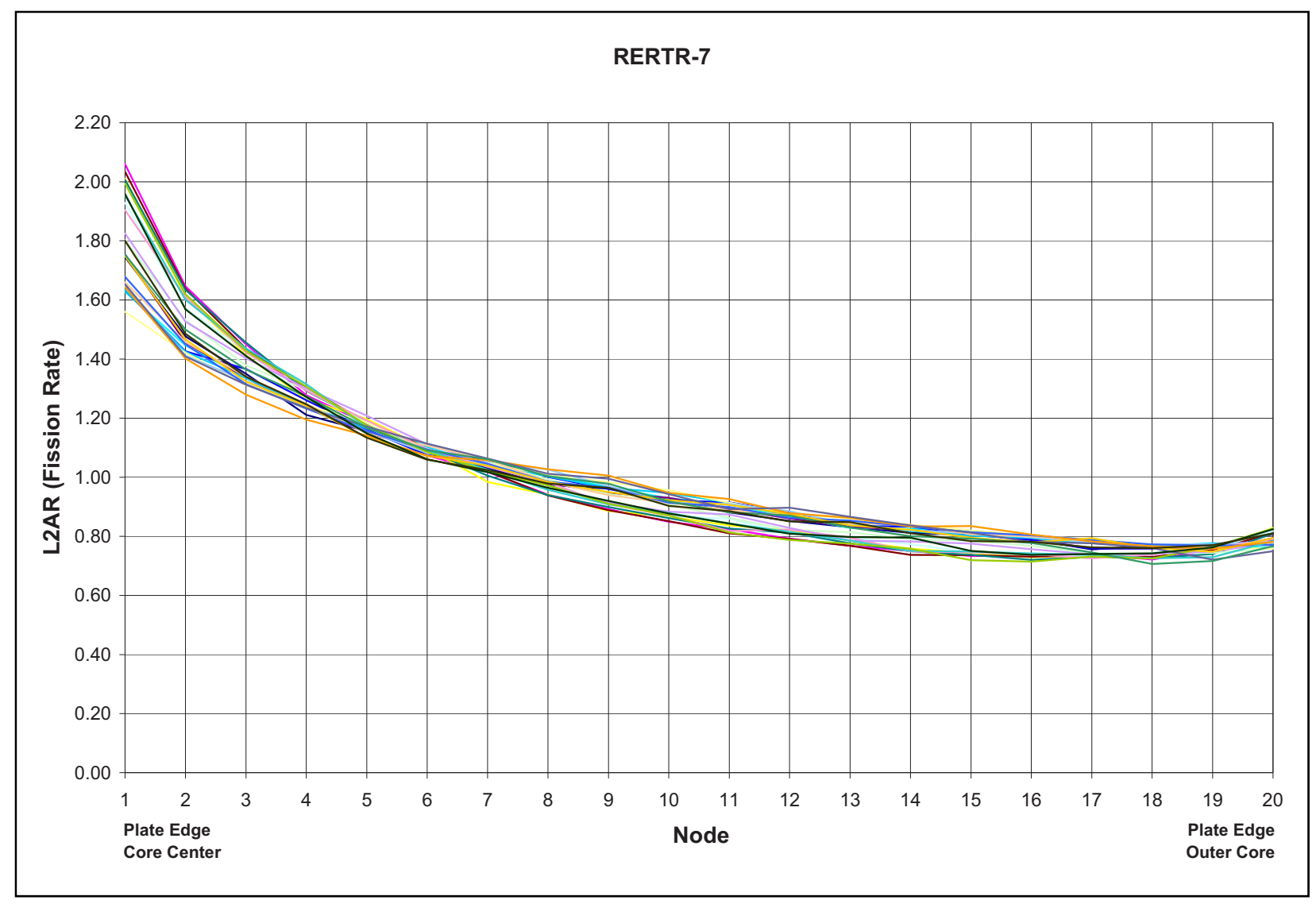

Figure 7. RERTR-7A fuel miniplates fission rate L2ARs in transverse direction. ${ }^{6}$ 


\section{HYDRAULIC TESTING}

A fully assembled irradiation test vehicle (with simulated fuel plates) was used for testing. The test vehicle was fabricated such that the orifice plates could be easily changed. The hydraulic resistance of the RERTR Large B-Position irradiation test vehicle with various orifice plate sizes were calculated, the results are shown in Table 19.

Table 19. Loss coefficients for the RERTR irradiation test vehicle components. ${ }^{7}$

\begin{tabular}{ccc}
\hline $\begin{array}{c}\text { Orifice Dia. } \\
(\mathrm{mm})\end{array}$ & $\begin{array}{c}\mathrm{K} / \mathrm{A}^{2} \\
\left(1 / \mathrm{m}^{4}\right)\end{array}$ & $\begin{array}{c}\text { ATR Coolant } \\
\text { Flow Rate } \\
\left(\mathrm{cm}^{3} / \mathrm{sec}\right)\end{array}$ \\
\hline 10 & $5.3041 \times 10^{8}$ & 1252 \\
9 & $8.2181 \times 10^{8}$ & 1046 \\
8 & $1.6961 \times 10^{9}$ & 757 \\
7.32 & $2.9022 \times 10^{9}$ & 588 \\
7 & $3.0058 \times 10^{9}$ & 579 \\
6 & $4.0784 \times 10^{9}$ & 500 \\
5 & $101743 \times 10^{10}$ & 298 \\
\hline Bypass & $2.7958 \times 10^{8}$ & -- \\
Vehicle & $1.4161 \times 10^{8}$ & 2727 \\
\hline
\end{tabular}

Based on the results from the hydraulic testing, the orifice was removed leaving the capsule in the "Vehicle" configuration to provide an ATR coolant flow rate through the capsules of $2727 \mathrm{~cm}^{3} / \mathrm{sec}^{8}$ 


\section{AS-RUN THERMAL ANALYSIS}

The thermal as-run analysis was performed using the as-built geometry, MCNP-calculated surface heat flux $\left(\mathrm{W} / \mathrm{cm}^{2}\right)$ and nominal coolant channel flow rate. ABAQUS ${ }^{9}$ was used to calculate the coolant channel temperatures and plate surface temperatures.

The heat transfer correlation used to calculate these temperatures was calculated from the Colburn equation (equation 5-50c from Reference 10):

$$
N u=\frac{h D}{k}=0.023 R e^{0.8} \operatorname{Pr}^{0.3}
$$

Where $\mathrm{Nu}$ is the Nusselt number, $\mathrm{h}$ is the heat transfer coefficient, $\mathrm{D}$ is the hydraulic diameter, $\mathrm{k}$ is the thermal conductivity, Re is the Reynolds number and Pr is the Prandlt number.

\subsection{Coolant Temperature as a Function of Location}

The coolant temperature was analyzed at the five flow channels in the test assembly, with Channel 1 at the right of the assembly. For each cycle, the coolant temperature was plotted as a function of location along the test assembly with 0 inches being at the top of the assembly. These plots are shown in Figure 8 through Figure 16.

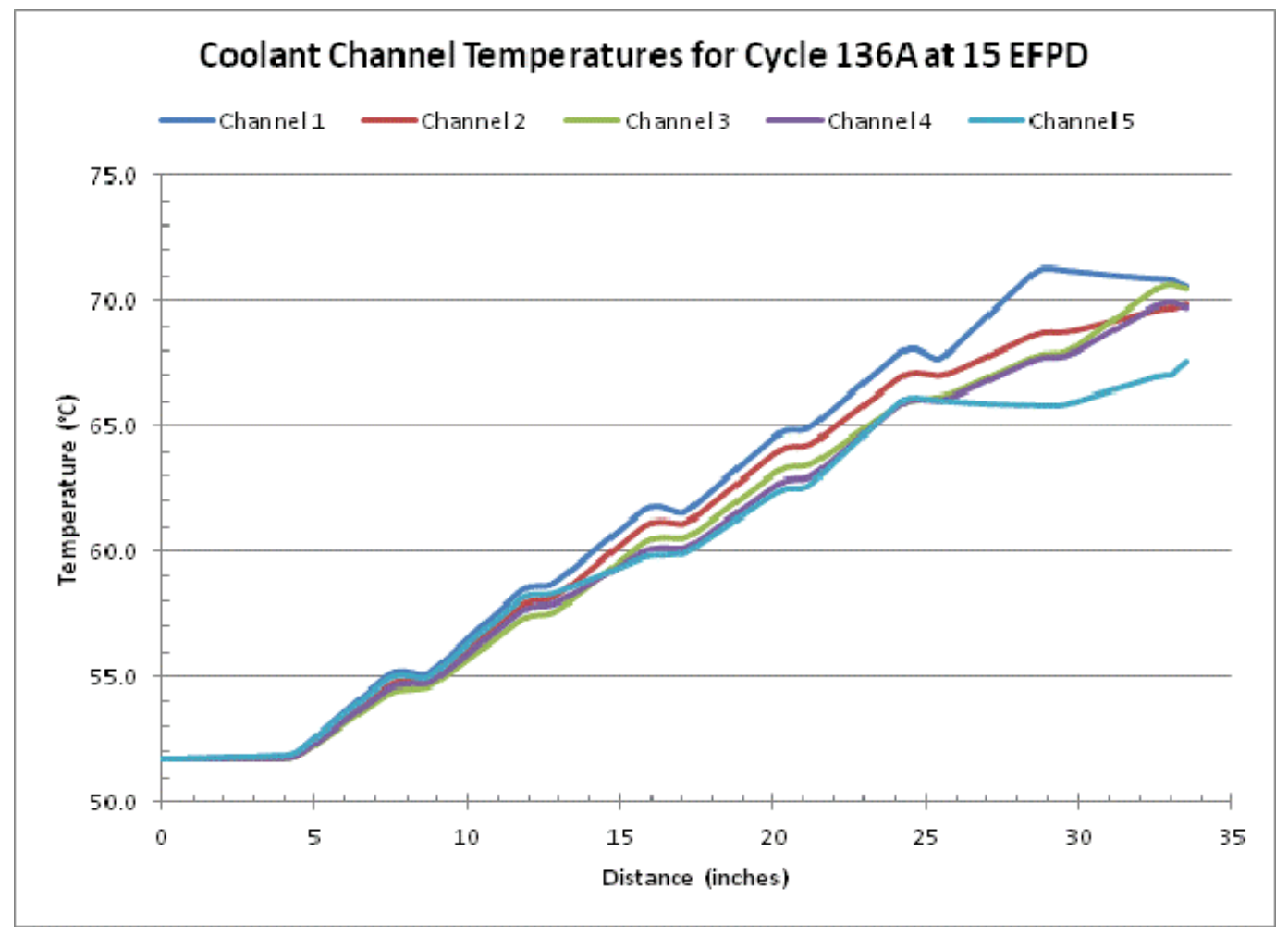

Figure 8. RERTR-7A coolant temperature as a function of location along the test assembly for Cycle $136 \mathrm{~A}$ at 15 EFPD. 


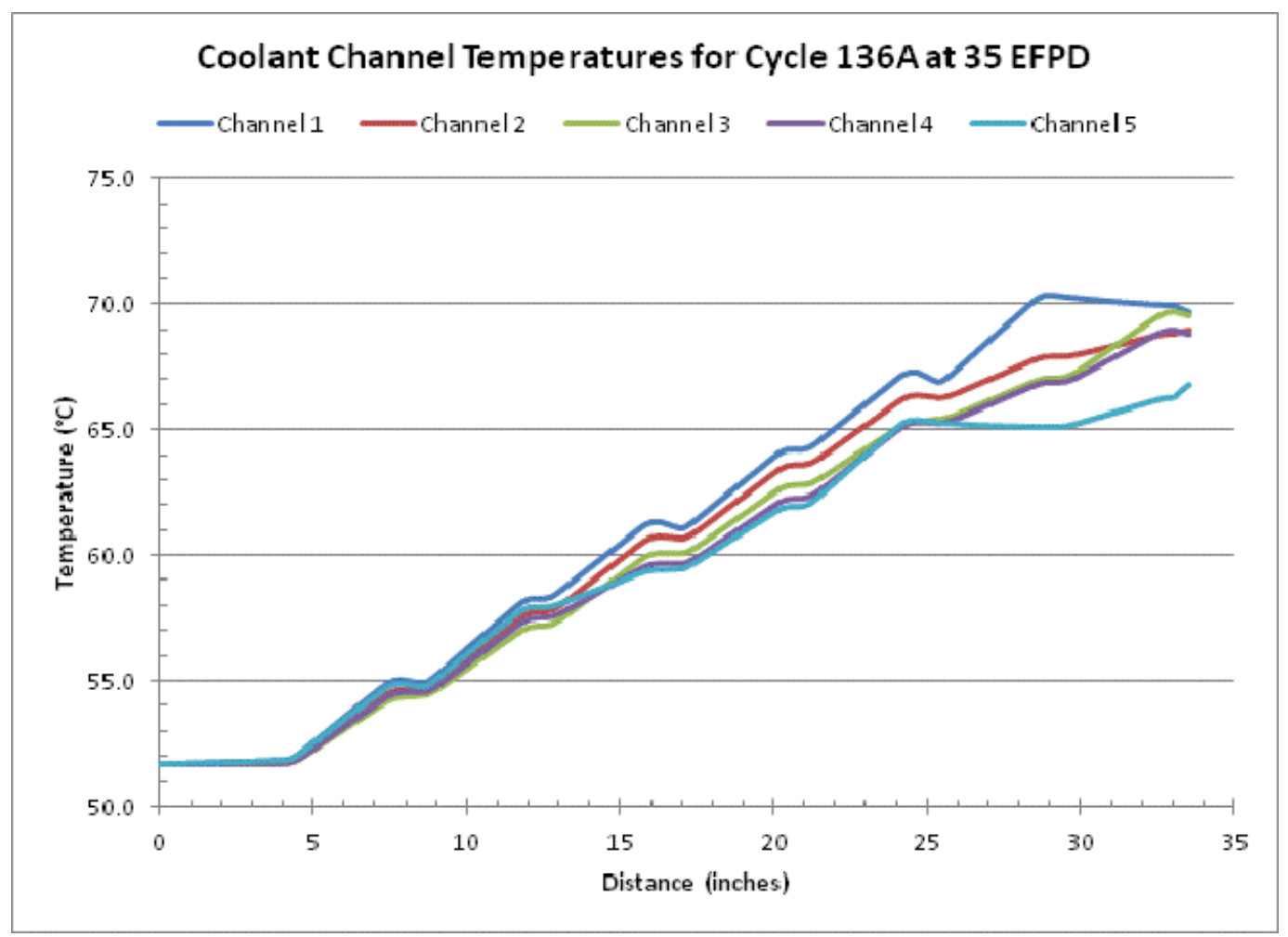

Figure 9. RERTR-7A coolant temperature as a function of location along the test assembly for Cycle $136 \mathrm{~A}$ at 18 EFPD.

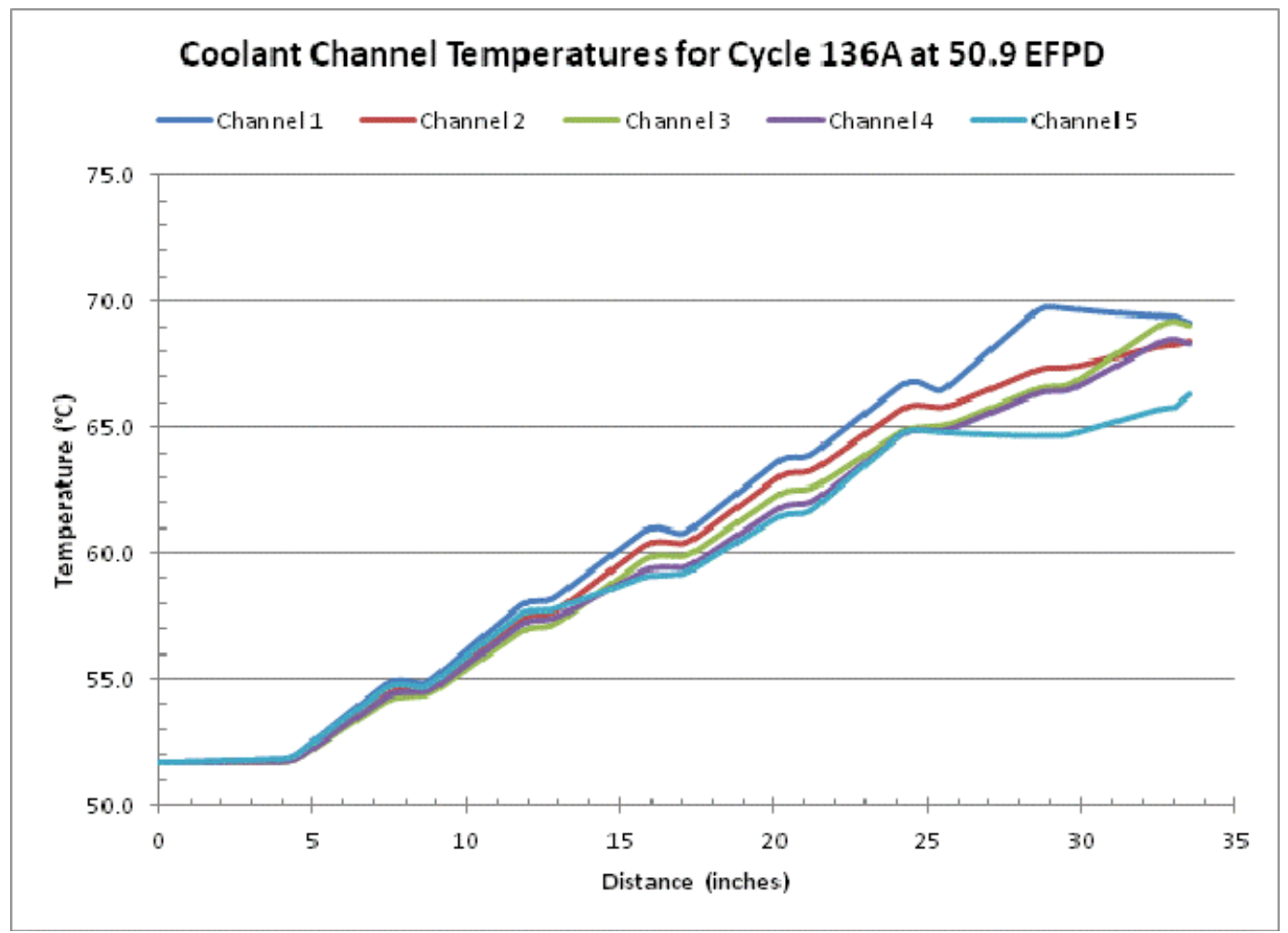

Figure 10. RERTR-7A coolant temperature as a function of location along the test assembly for Cycle 136A at 50.9 EFPD. 


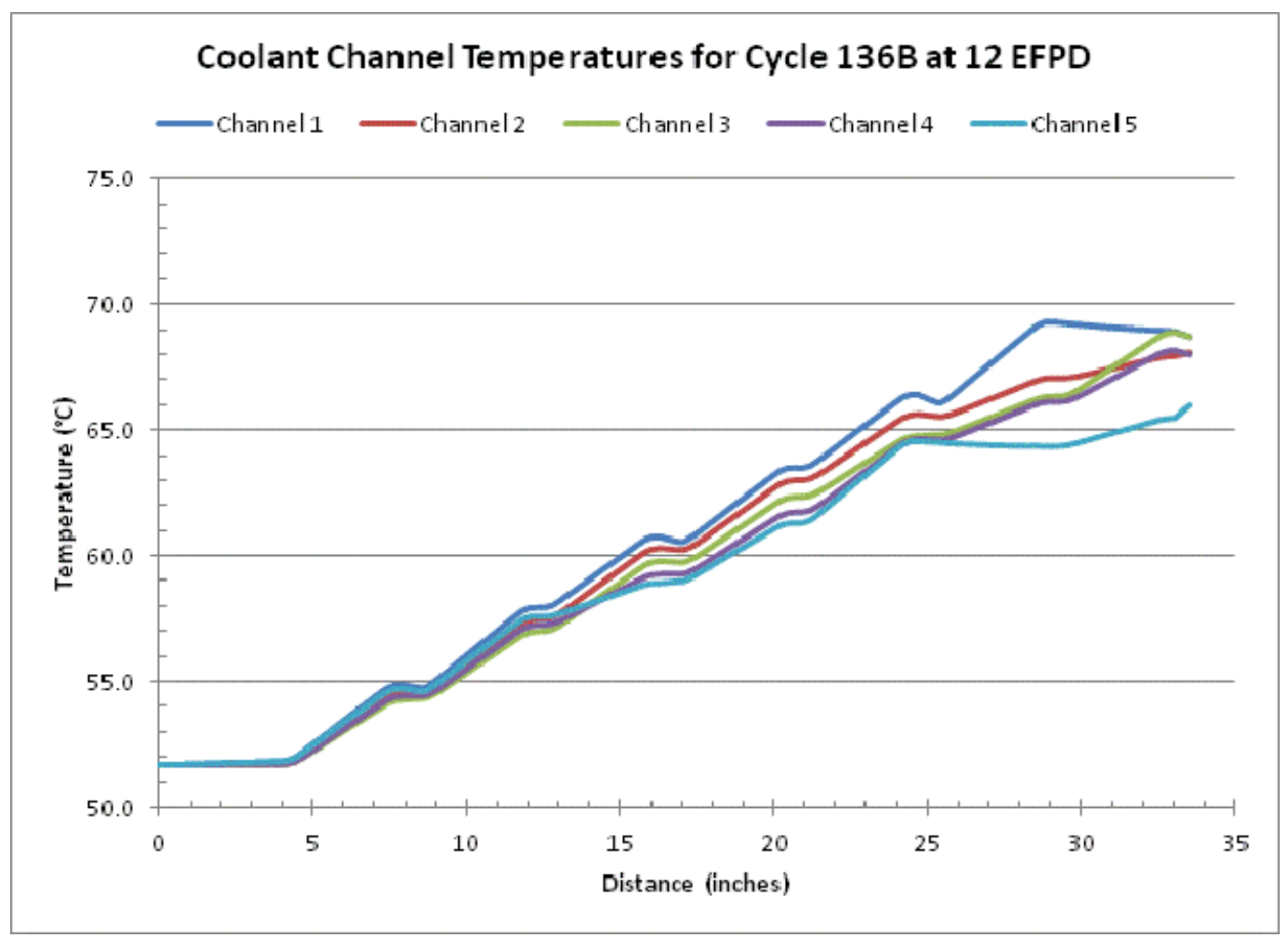

Figure 11. RERTR-7A coolant temperature as a function of location along the test assembly for Cycle $146 \mathrm{~B}$ at $12 \mathrm{EFPD}$.

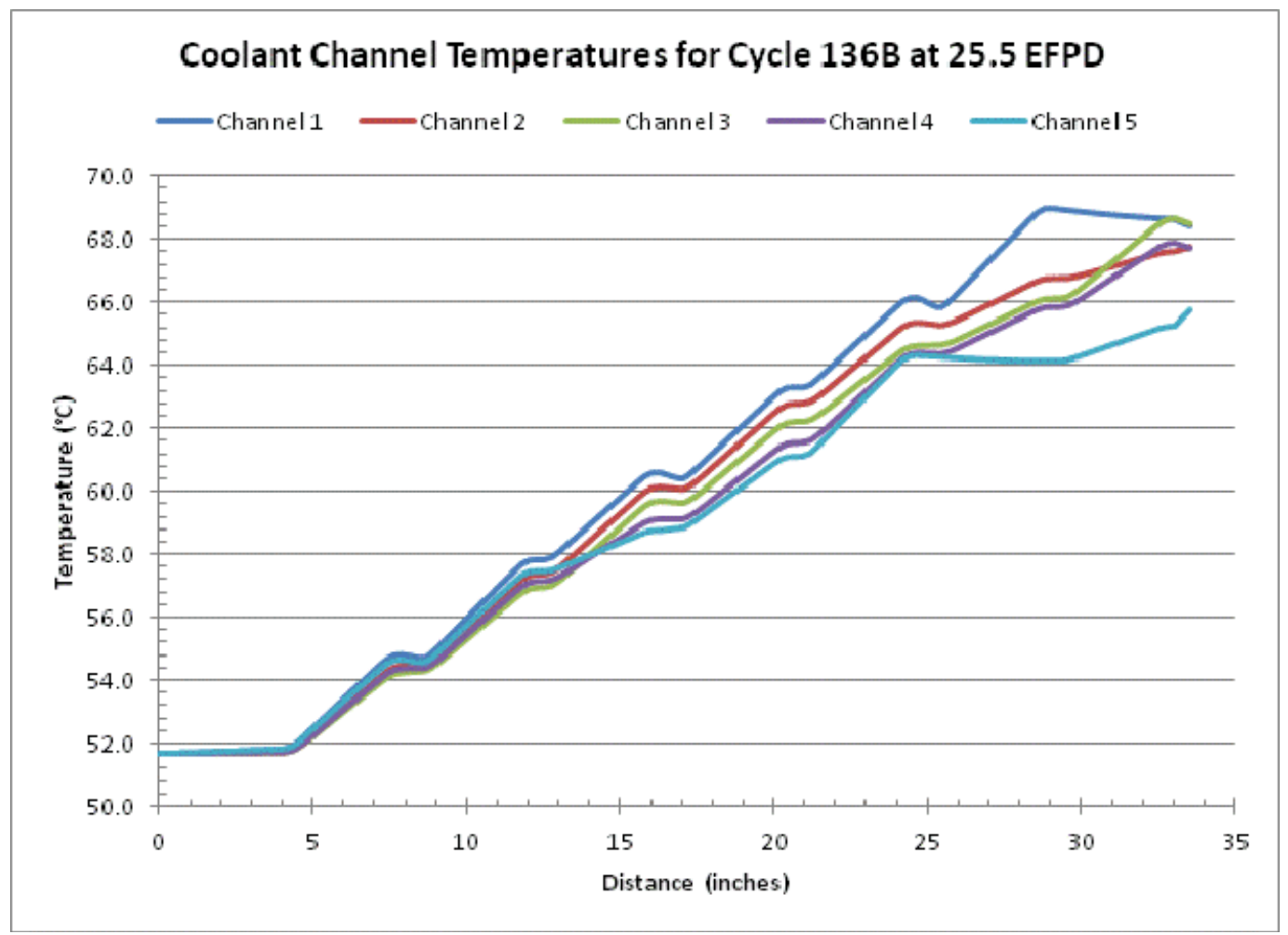

Figure 12. RERTR-7A coolant temperature as a function of location along the test assembly for Cycle $146 \mathrm{~B}$ at $25.5 \mathrm{EFPD}$. 


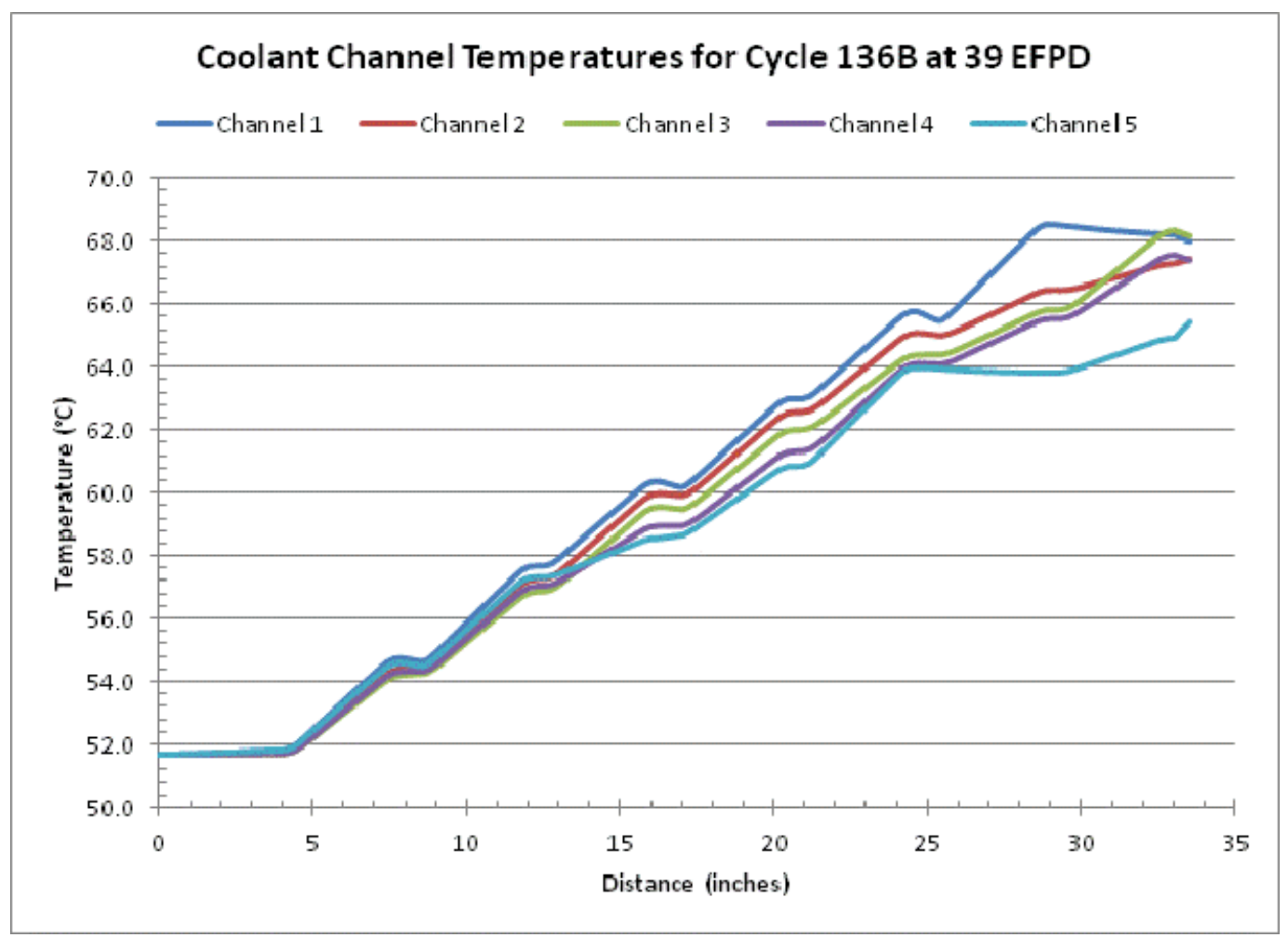

Figure 13. RERTR-7A coolant temperature as a function of location along the test assembly for Cycle $146 \mathrm{~B}$ at $39 \mathrm{EFPD}$.

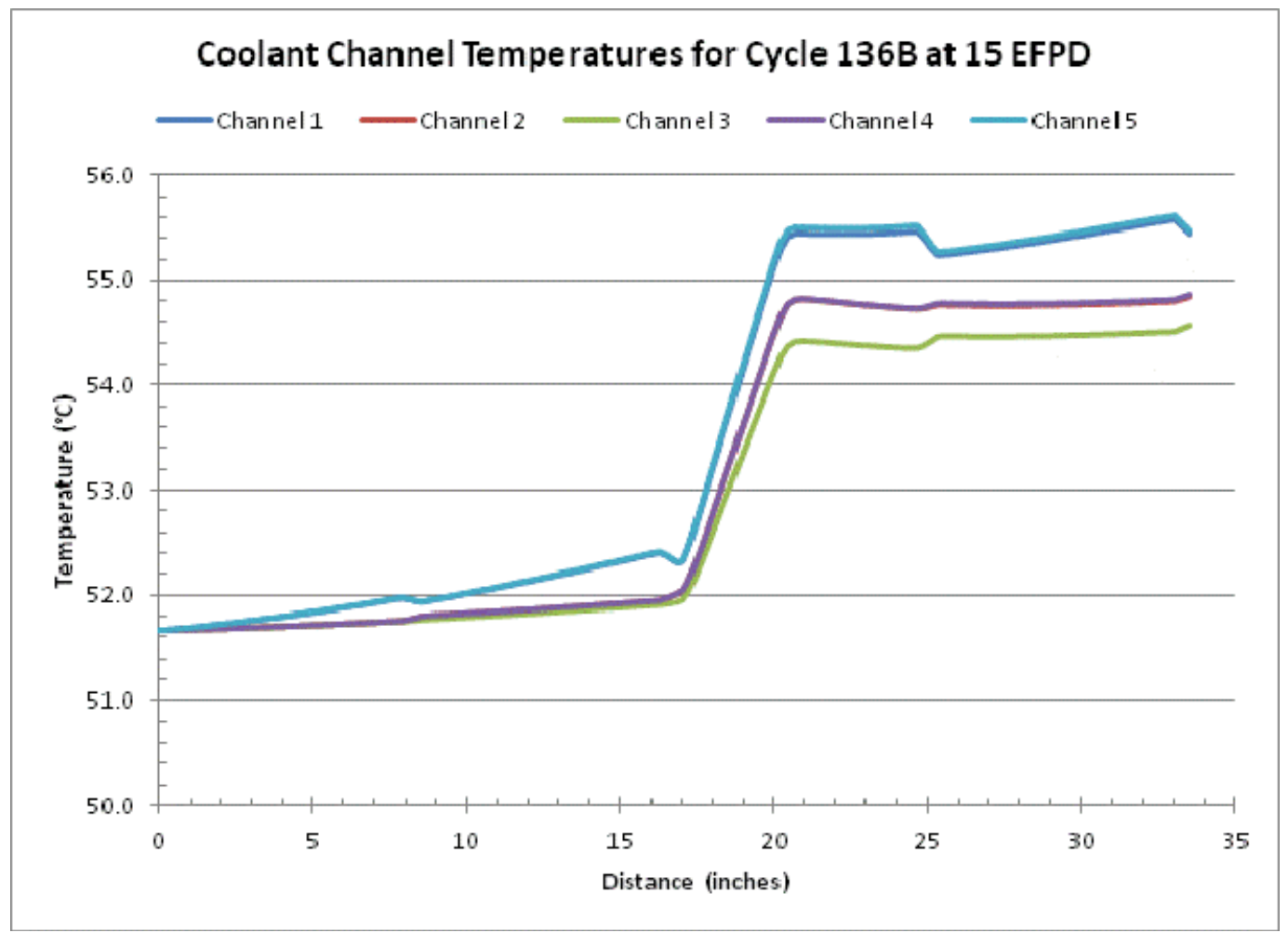

Figure 14. RERTR-7B coolant temperature as a function of location along the test assembly for Cycle $146 \mathrm{~B}$ at 15 EFPD. 


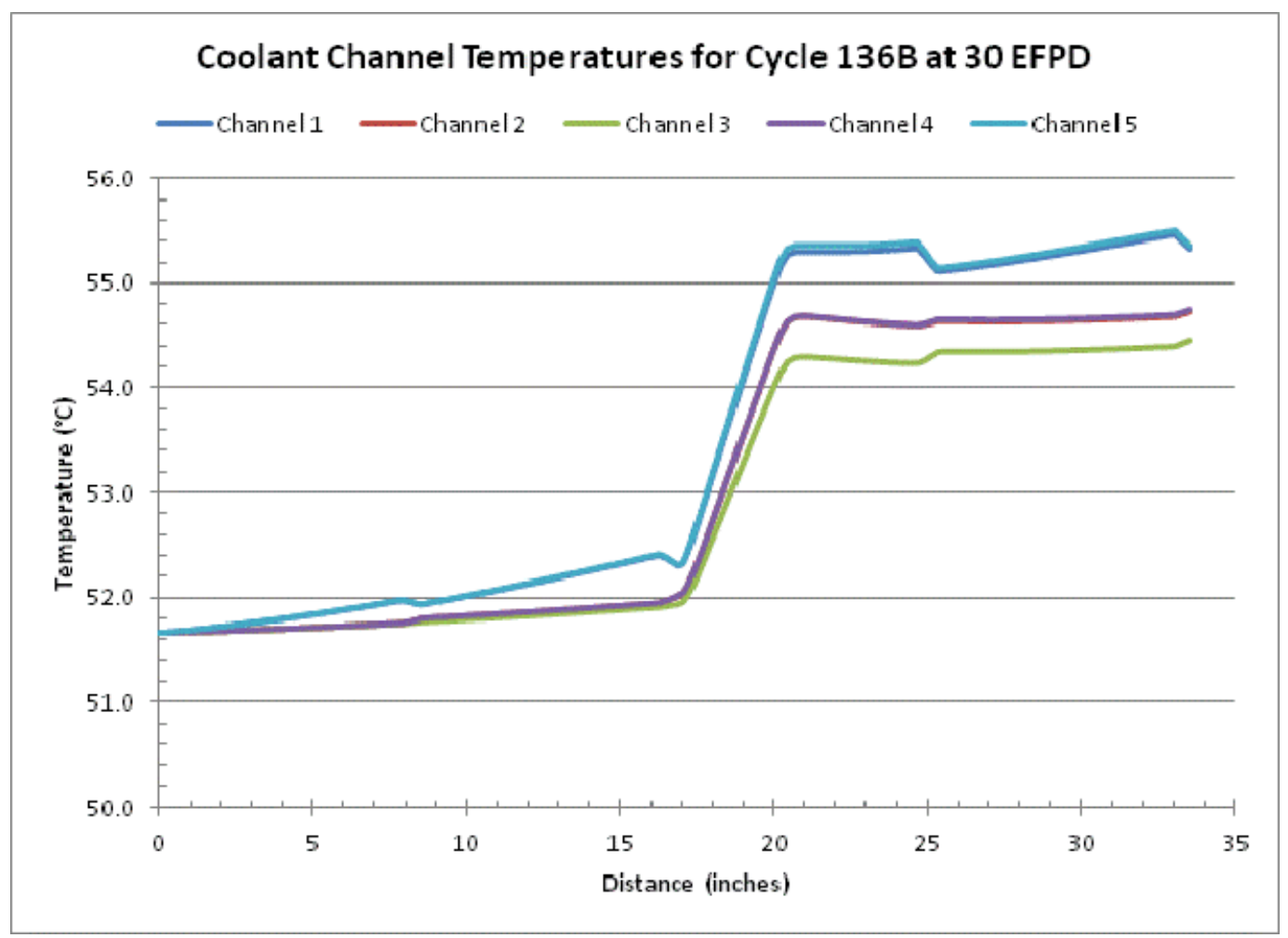

Figure 15. RERTR-7B coolant temperature as a function of location along the test assembly for Cycle $146 \mathrm{~B}$ at $30 \mathrm{EFPD}$.

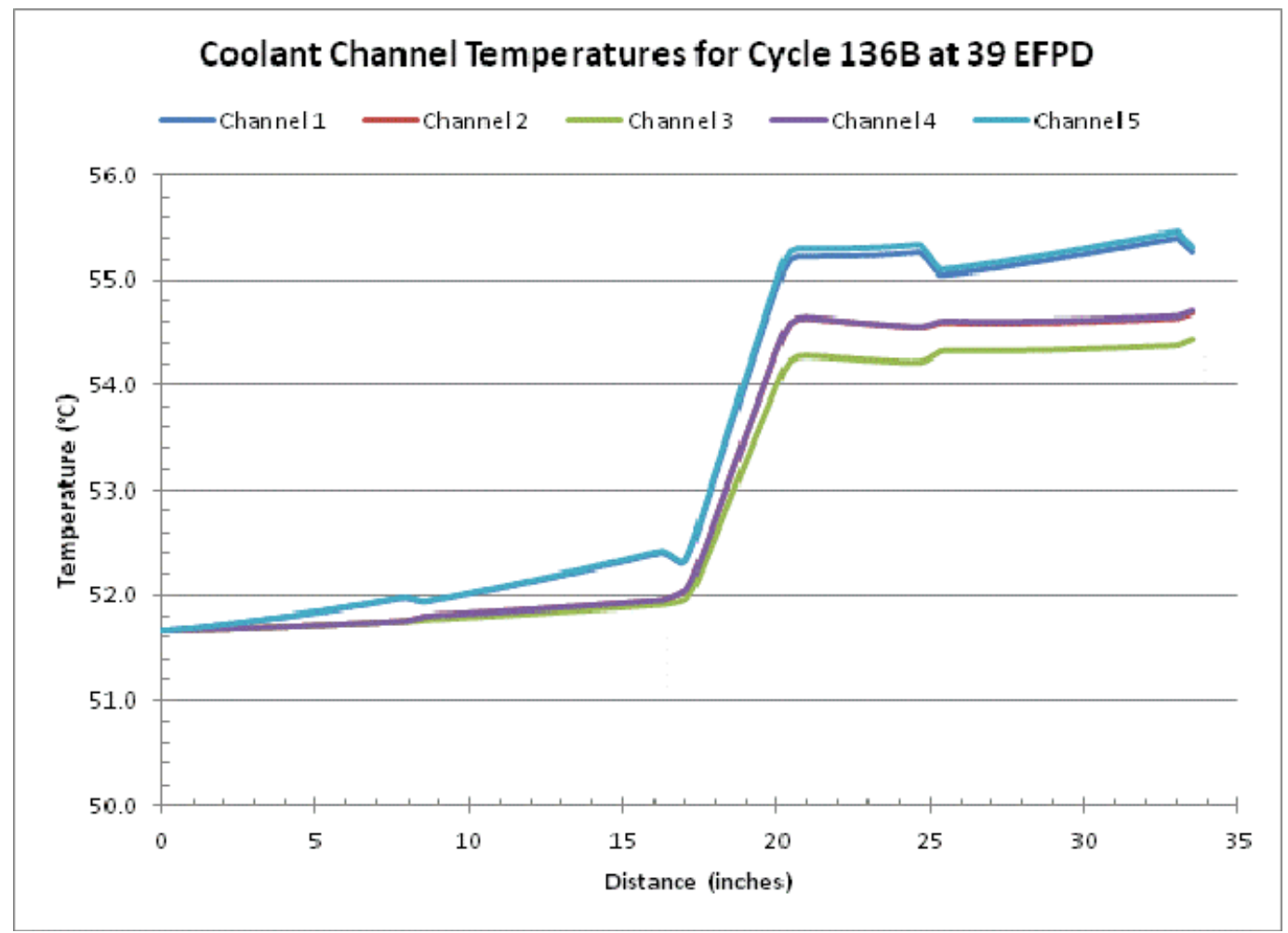

Figure 16. RERTR-7B coolant temperature as a function of location along the test assembly for Cycle $146 \mathrm{~B}$ at $39 \mathrm{EFPD}$. 


\subsection{Plate Surface Temperature}

The maximum, minimum, and average plate temperatures for each cycle are provided in Table 20 through Error! Reference source not found..

Table 20. RERTR-7A plate surface temperatures for Cycle 136A (50.9 EFPD).

\begin{tabular}{|c|c|c|c|c|}
\hline $\begin{array}{c}\text { Plate } \\
\text { Location }\end{array}$ & Plate ID & $\begin{array}{c}\text { Maximum } \\
\text { Temp }\left({ }^{\circ} \mathrm{C}\right)\end{array}$ & $\begin{array}{c}\text { Minimum } \\
\text { Temp }\left({ }^{\circ} \mathrm{C}\right)\end{array}$ & $\begin{array}{c}\text { Average } \\
\text { Temp }\left({ }^{\circ} \mathrm{C}\right)\end{array}$ \\
\hline \hline A1 & Blank & -- & -- & -- \\
\hline A2 & Blank & -- & -- & -- \\
\hline A3 & Blank & -- & -- & -- \\
\hline A4 & Blank & -- & -- & -- \\
\hline A5 & R3R040 & 99.65 & 48.46 & 80.76 \\
\hline A6 & V5R040 & 91.01 & 49.00 & 76.02 \\
\hline A7 & R5R030 & 91.19 & 48.97 & 76.15 \\
\hline A8 & H1F020 & 96.67 & 48.72 & 78.92 \\
\hline B1 & R1R040 & 106.59 & 53.87 & 84.86 \\
\hline B2 & R2R040 & 96.84 & 53.72 & 79.71 \\
\hline B3 & R0R010 & 96.85 & 53.70 & 79.75 \\
\hline B4 & H1T010 & 102.76 & 53.85 & 82.65 \\
\hline B5 & L1F01L & 105.84 & 54.13 & 86.39 \\
\hline B6 & V5R050 & 101.97 & 53.71 & 84.61 \\
\hline B7 & L1F140 & 101.17 & 53.69 & 84.15 \\
\hline B8 & MZ25 & 82.92 & 55.63 & 72.53 \\
\hline C1 & H1F030 & 110.08 & 59.58 & 89.97 \\
\hline C2 & L1T020 & 101.62 & 59.35 & 85.29 \\
\hline C3 & L1F110 & 102.57 & 58.69 & 85.79 \\
\hline C4 & MZ 50 & 97.26 & 57.81 & 82.01 \\
\hline C5 & L1F120 & 112.43 & 60.63 & 92.86 \\
\hline C6 & H1T020 & 100.95 & 60.45 & 86.19 \\
\hline C7 & R3R050 & 103.55 & 59.71 & 87.82 \\
\hline C8 & R5R040 & 112.54 & 58.96 & 92.68 \\
\hline D1 & R1R050 & 119.19 & 63.73 & 97.29 \\
\hline D2 & Blank & -- & -- & -- \\
\hline D3 & R0R020 & 114.18 & 62.51 & 94.96 \\
\hline D4 & Blank & -- & -- & -- \\
\hline D5 & Blank & -- & -- & -- \\
\hline D6 & L1F160 & 98.59 & 64.89 & 86.62 \\
\hline D7 & L2F040 & 113.96 & 63.95 & 96.52 \\
\hline D8 & R2R050 & 83.42 & 64.32 & 76.37 \\
\hline & & & & \\
\hline
\end{tabular}


Table 21. RERTR-7A plate surface temperatures for Cycle 136B (39 EFPD).

\begin{tabular}{|c|c|c|c|c|}
\hline $\begin{array}{c}\text { Plate } \\
\text { Location }\end{array}$ & Plate ID & $\begin{array}{c}\text { Maximum } \\
\text { Temp }\left({ }^{\circ} \mathrm{C}\right)\end{array}$ & $\begin{array}{c}\text { Minimum } \\
\text { Temp }\left({ }^{\circ} \mathrm{C}\right)\end{array}$ & $\begin{array}{c}\text { Average } \\
\text { Temp }\left({ }^{\circ} \mathrm{C}\right)\end{array}$ \\
\hline \hline A1 & Blank & -- & -- & -- \\
\hline A2 & Blank & -- & -- & -- \\
\hline A3 & Blank & -- & -- & -- \\
\hline A4 & Blank & -- & -- & -- \\
\hline A5 & R3R040 & 97.13 & 48.68 & 79.20 \\
\hline A6 & V5R040 & 89.85 & 49.09 & 75.29 \\
\hline A7 & R5R030 & 90.02 & 49.07 & 75.42 \\
\hline A8 & H1F020 & 94.17 & 48.94 & 77.37 \\
\hline \hline B1 & R1R040 & 103.26 & 53.82 & 82.90 \\
\hline B2 & R2R040 & 95.42 & 53.65 & 78.82 \\
\hline B3 & R0R010 & 95.31 & 53.66 & 78.78 \\
\hline B4 & H1T010 & 99.11 & 53.82 & 80.52 \\
\hline B5 & L1F01L & 102.12 & 54.11 & 84.07 \\
\hline B6 & V5R050 & 100.01 & 53.64 & 83.37 \\
\hline B7 & L1F140 & 98.83 & 53.65 & 82.62 \\
\hline B8 & MZ25 & 79.76 & 55.53 & 70.51 \\
\hline \hline C1 & H1F030 & 106.13 & 59.19 & 87.43 \\
\hline C2 & L1T020 & 99.61 & 58.96 & 83.95 \\
\hline C3 & L1F110 & 100.37 & 58.28 & 84.28 \\
\hline C4 & MZ 50 & 93.71 & 57.41 & 79.74 \\
\hline C5 & L1F120 & 108.48 & 60.17 & 90.22 \\
\hline C6 & H1T020 & 98.86 & 60.03 & 84.74 \\
\hline C7 & R3R050 & 101.70 & 59.23 & 86.45 \\
\hline C8 & R5R040 & 108.86 & 58.48 & 90.21 \\
\hline \hline D1 & R1R050 & 114.62 & 63.09 & 94.25 \\
\hline D2 & Blank & -- & -- & -- \\
\hline D3 & R0R020 & 110.04 & 61.86 & 92.11 \\
\hline D4 & Blank & -- & -- & -- \\
\hline D5 & Blank & -- & -- & -- \\
\hline D6 & L1F160 & 96.07 & 64.20 & 84.71 \\
\hline D7 & L2F040 & 112.68 & 63.13 & 95.42 \\
\hline D8 & R2R050 & 81.78 & 63.55 & 75.04 \\
\hline
\end{tabular}


Table 22. RERTR-7B plate surface temperatures for Cycle 136B (39 EFPD).

\begin{tabular}{|c|c|c|c|c|}
\hline $\begin{array}{c}\text { Plate } \\
\text { Location }\end{array}$ & Plate ID & $\begin{array}{c}\text { Maximum } \\
\text { Temp }\left({ }^{\circ} \mathrm{C}\right)\end{array}$ & $\begin{array}{c}\text { Minimum } \\
\text { Temp }\left({ }^{\circ} \mathrm{C}\right)\end{array}$ & $\begin{array}{c}\text { Average } \\
\text { Temp }\left({ }^{\circ} \mathrm{C}\right)\end{array}$ \\
\hline \hline A1 & Blank & -- & -- & -- \\
\hline A2 & Blank & -- & -- & -- \\
\hline A3 & Blank & -- & -- & -- \\
\hline A4 & Blank & -- & -- & -- \\
\hline A5 & Blank & -- & -- & -- \\
\hline A6 & Blank & -- & -- & -- \\
\hline A7 & Blank & -- & -- & -- \\
\hline A8 & Blank & -- & -- & -- \\
\hline \hline B1 & Blank & -- & -- & -- \\
\hline B2 & Blank & -- & -- & -- \\
\hline B3 & Blank & -- & -- & -- \\
\hline B4 & Blank & -- & -- & -- \\
\hline B5 & Blank & -- & -- & -- \\
\hline B6 & Blank & -- & -- & -- \\
\hline B7 & Blank & -- & -- & -- \\
\hline B8 & Blank & -- & -- & 79.32 \\
\hline \hline C1 & F3R010 & 96.53 & 51.74 & 74.42 \\
\hline C2 & R0R010 & 87.85 & 51.85 & 74.29 \\
\hline C3 & R3R010 & 87.65 & 51.86 & --35 \\
\hline C4 & D3R010 & 96.62 & 51.76 & -- \\
\hline C5 & Blank & -- & -- & -- \\
\hline C6 & Blank & -- & -- & -- \\
\hline C7 & Blank & -- & -- & -- \\
\hline C8 & Blank & -- & -- & -- \\
\hline \hline D1 & Blank & -- & -- & - \\
\hline D2 & Blank & -- & -- & - \\
\hline D3 & Blank & -- & -- & - \\
\hline D4 & Blank & -- & - & - \\
\hline D5 & Blank & -- & - & - \\
\hline D6 & Blank & -- & - & - \\
\hline D7 & Blank & -- & - & - \\
\hline D8 & Blank & -- & - & - \\
\hline
\end{tabular}




\section{REFERENCES}

1. Wachs, D. M. "RERTR Fuel Development and Qualification Plan," INL/EXT-05-01017 Rev 4, August 2009.

2. RERTR Project Personnel, "RERTR-7A Irradiation Experiments in the Advanced Test Reactor: AsBuilt Data Package" RERTR-7A, August 2006.

3. RERTR Project Personnel, "RERTR 7B-C Irradiation Experiments in the Advanced Test Reactor," RERTR-7B-C, January 2006.

4. M. A. Lillo, G. S. Chang, "RERTR-7A As-Run Physics Analysis and Test Train Isotopes Radiological Characterization Versus Cooling Time," EDF-6857 Rev 2, January 2007.

5. M. A. Lillo, G. S. Chang, "RERTR-7B Capsule C As-Run Physics Analysis and Test Train Isotopes Radiological Characterization Versus Cooling Time,” EDF-6919, June 2006.

6. M. A. Lillo to M. R. Finlay, "MCNP-Calculated Gradients Across RERTR-6 and RERTR-7A Miniplates Irradiated in ATR," Interoffice Memorandum, January 2007. (See appendix B)

7. Wachs, D. M., 2007, "RERTR Large B Position Irradiation Vehicle Flow Test,” EDF-8292, July 2007.

8. Wachs, D. M., 2007, "Estimation of Critical Thermal Hydraulic Conditions for RERTR-7," EDF-5671, February 2006.

9. P. E. Murray, "Validation of ABAQUS Standard 6.7-3 Heat Transfer," ECAR-131, January 2008.

10. R. H. Perry, D. W. Green, "Perry's Chemical Engineer's Handbook," $7^{\text {th }}$ Edition, McGraw-Hill, 1997. 


\section{Appendix A}

\section{Individual Plate Power and Burnup Plots}




\section{Appendix A \\ Individual Plate Power and Burnup Plots}

\section{A-1. RERTR-7A}

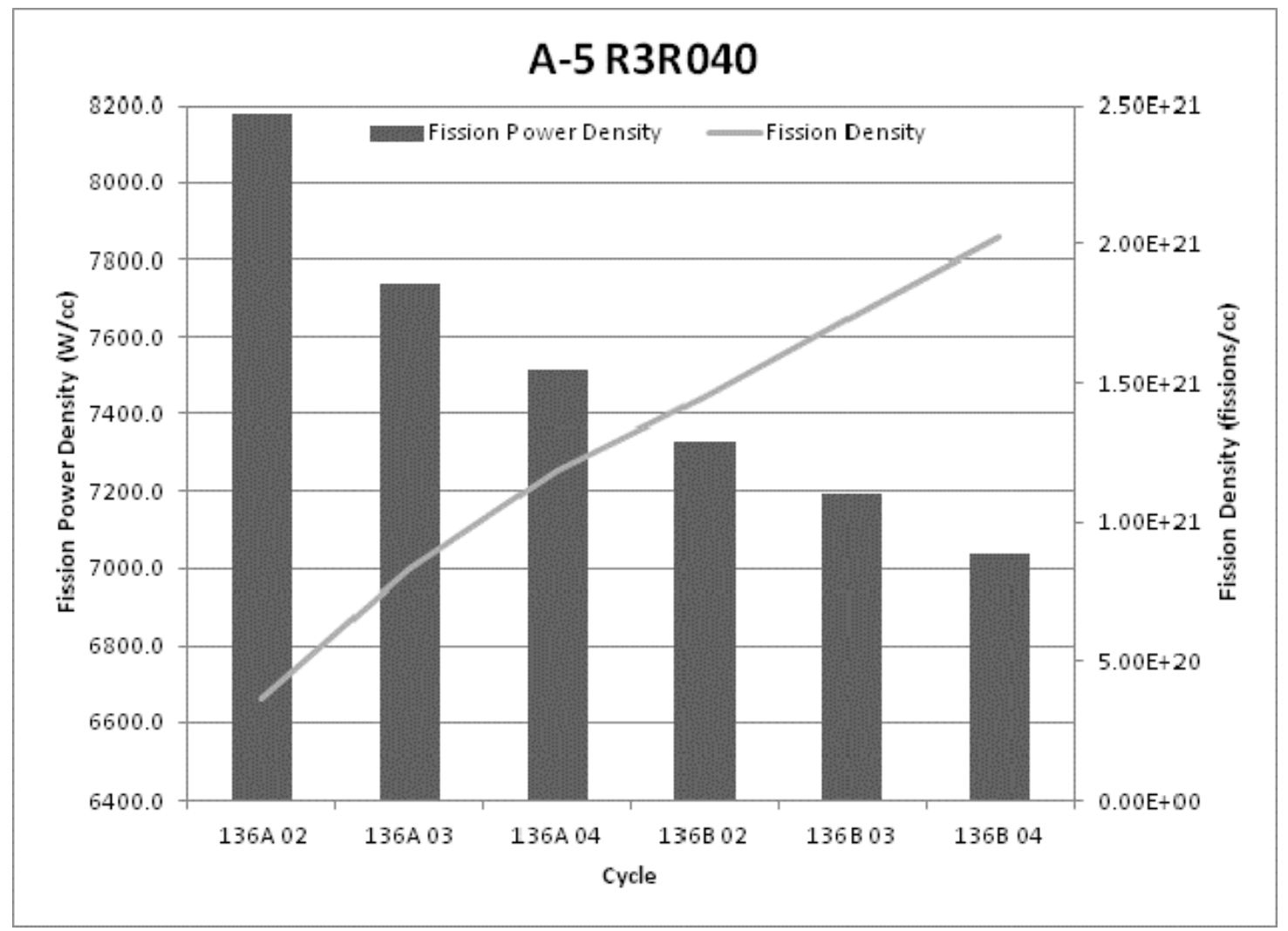



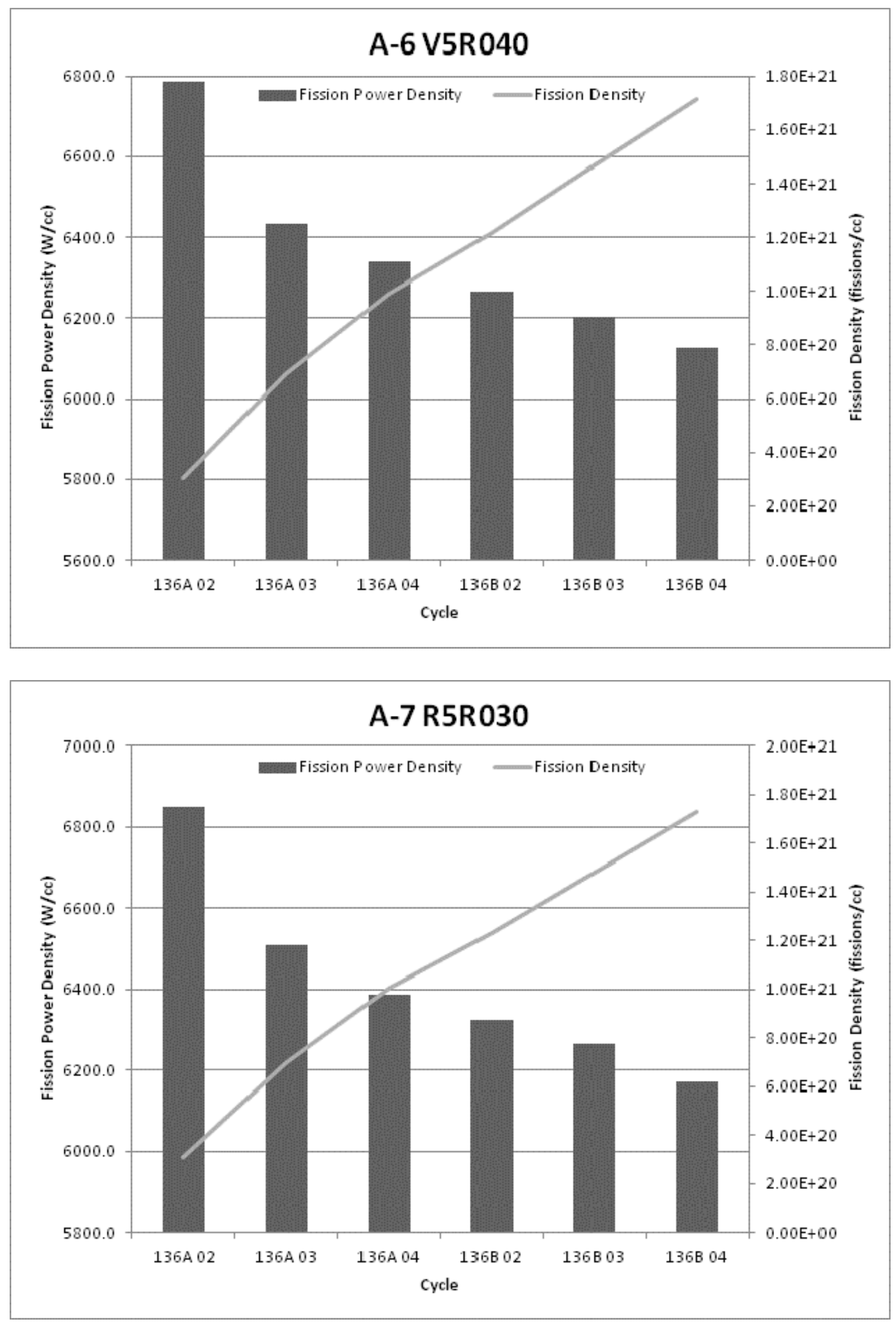

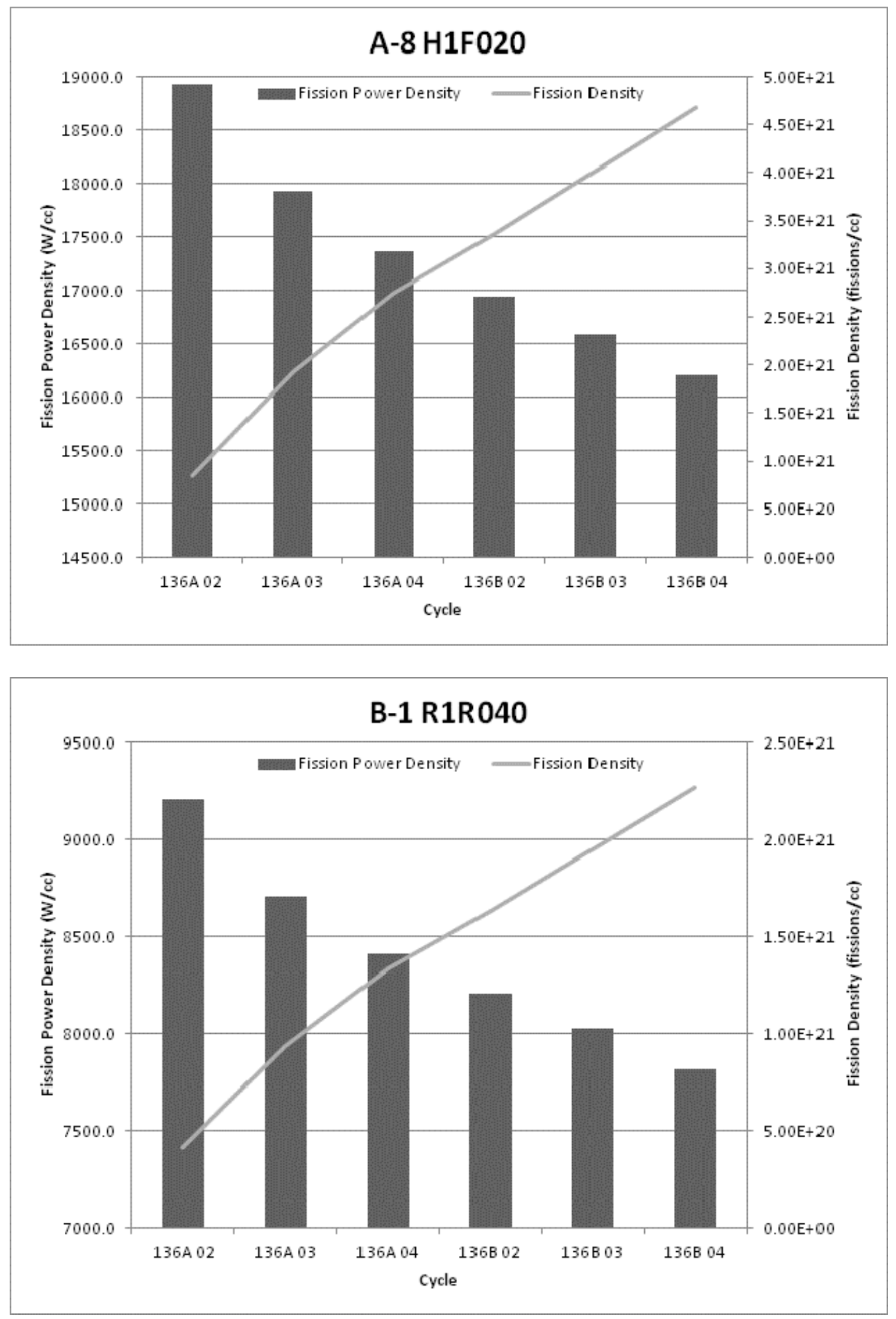

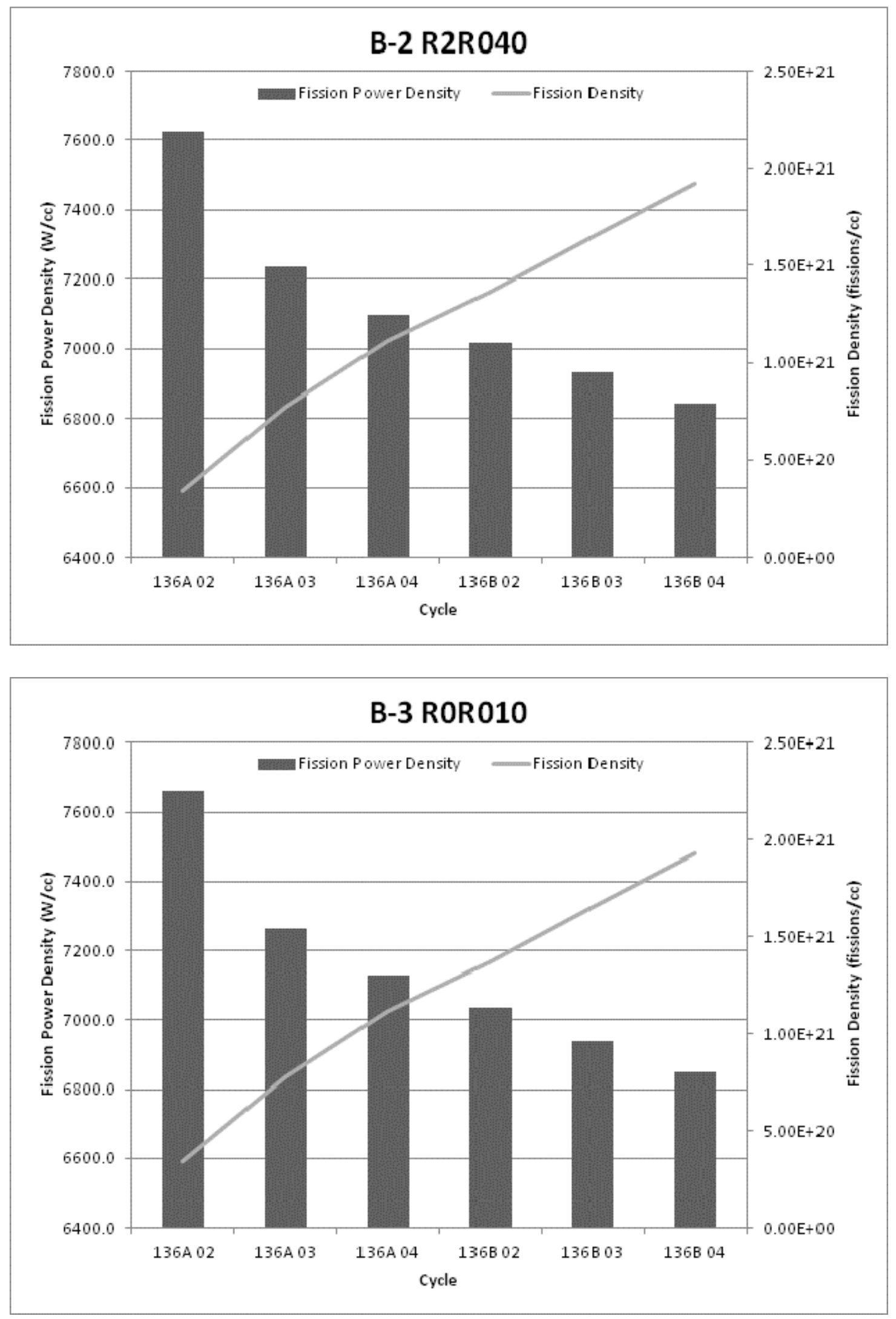

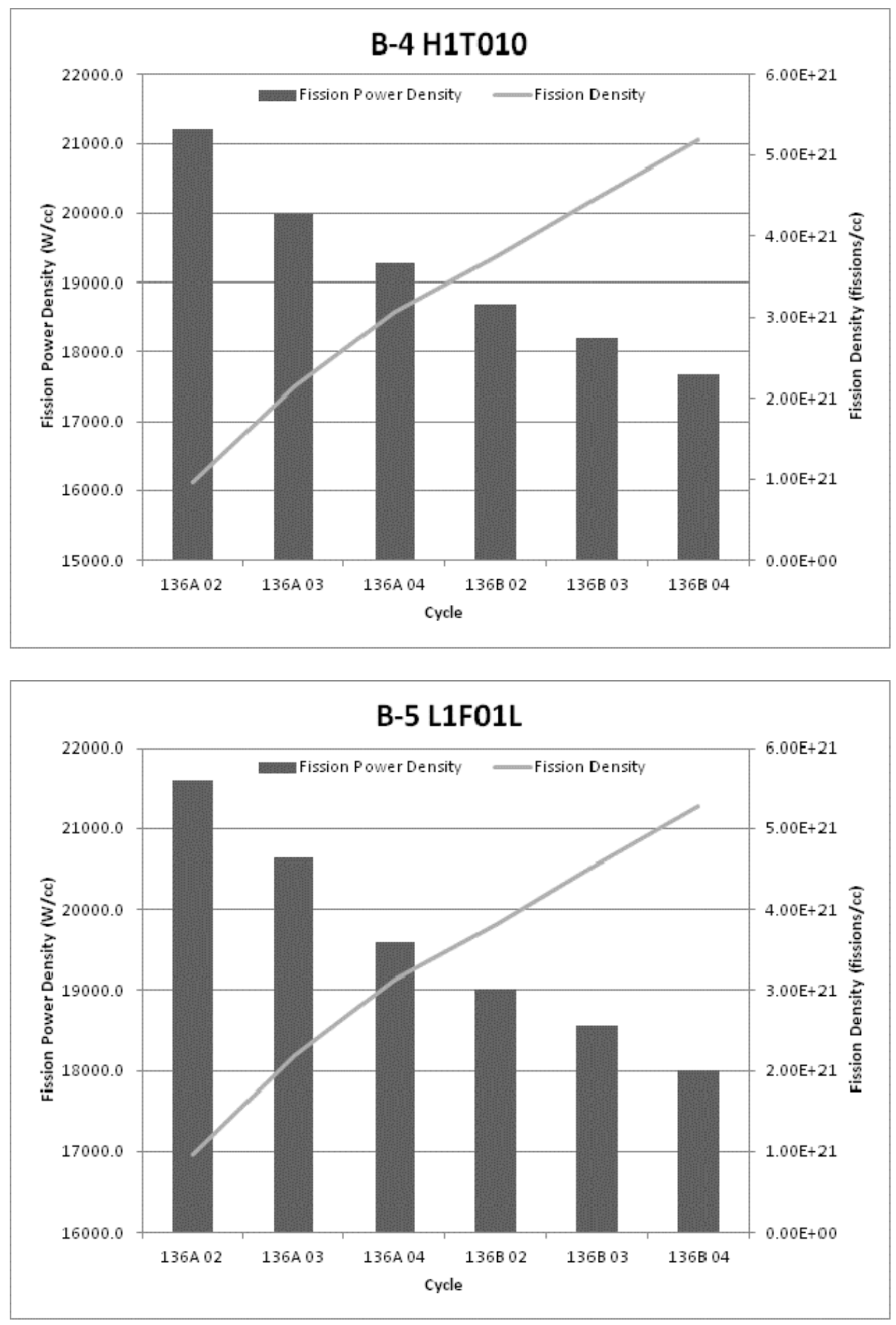

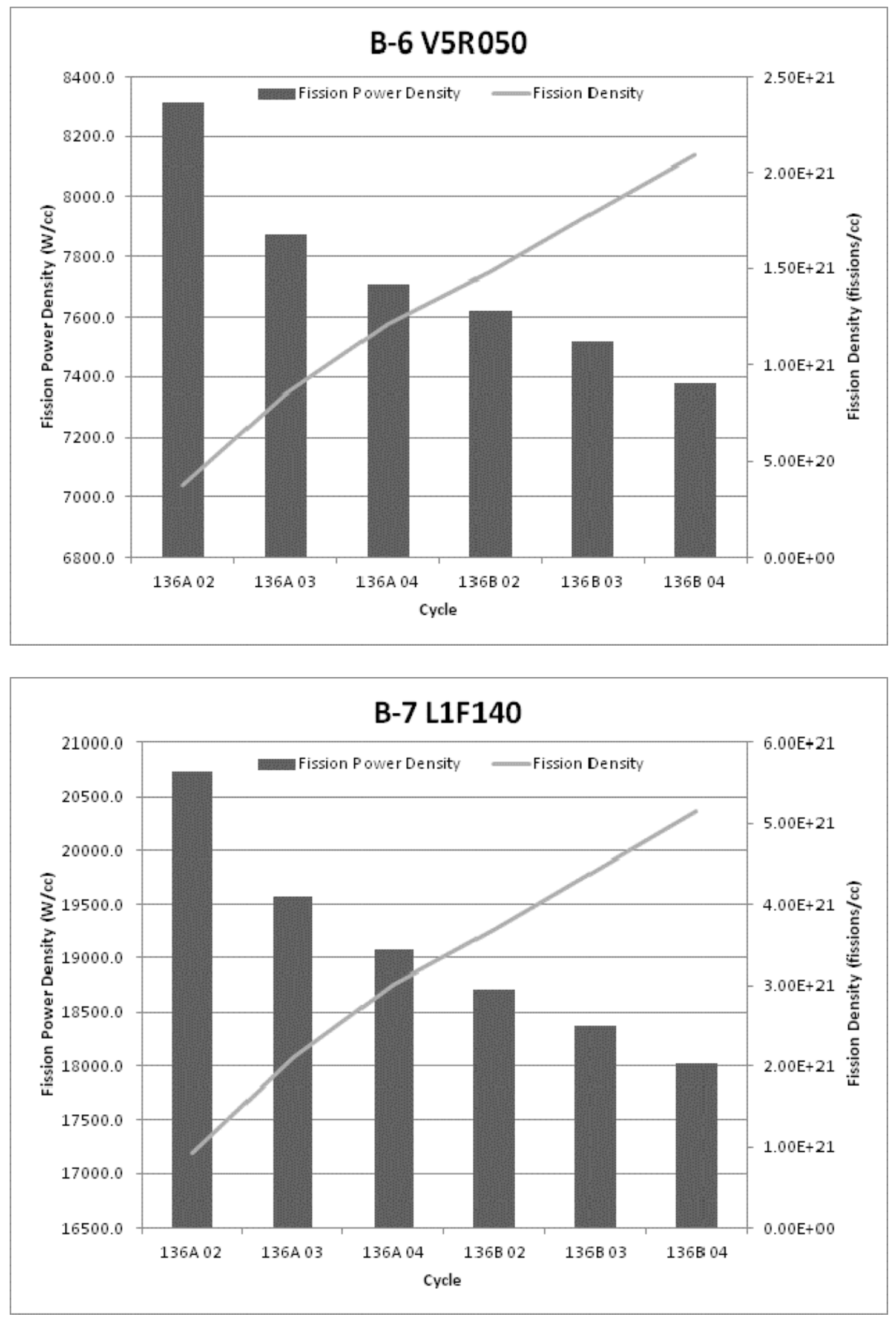

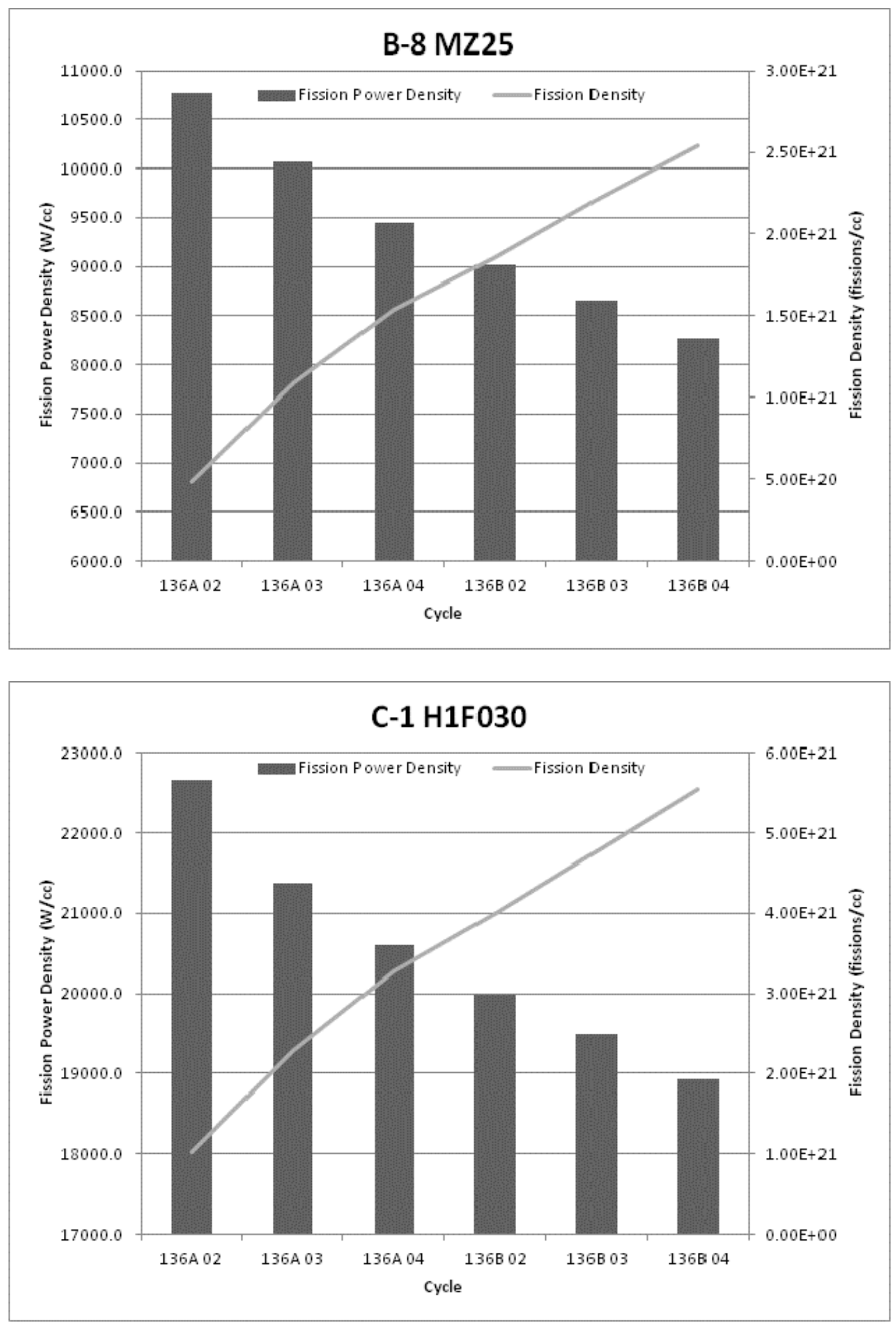

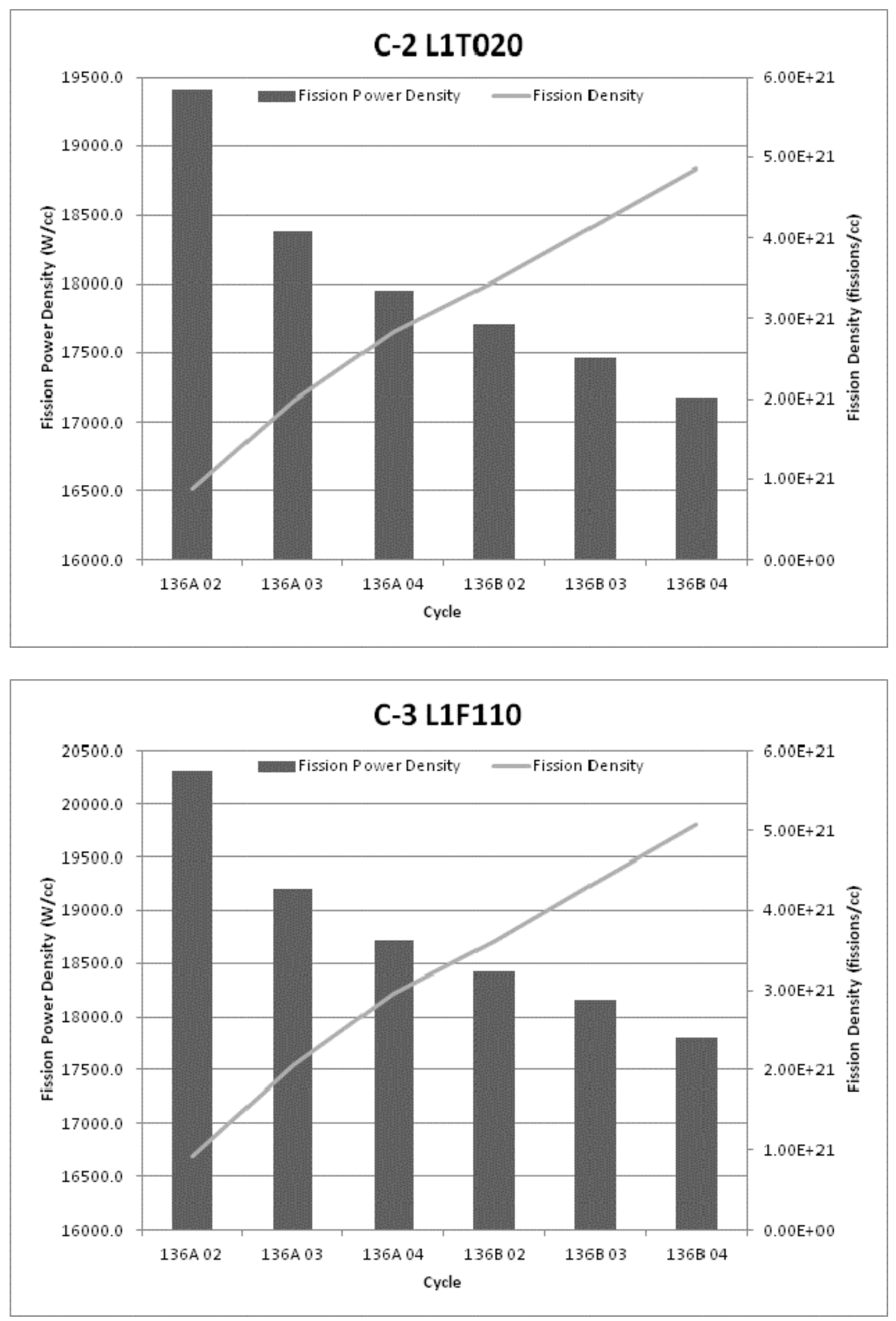

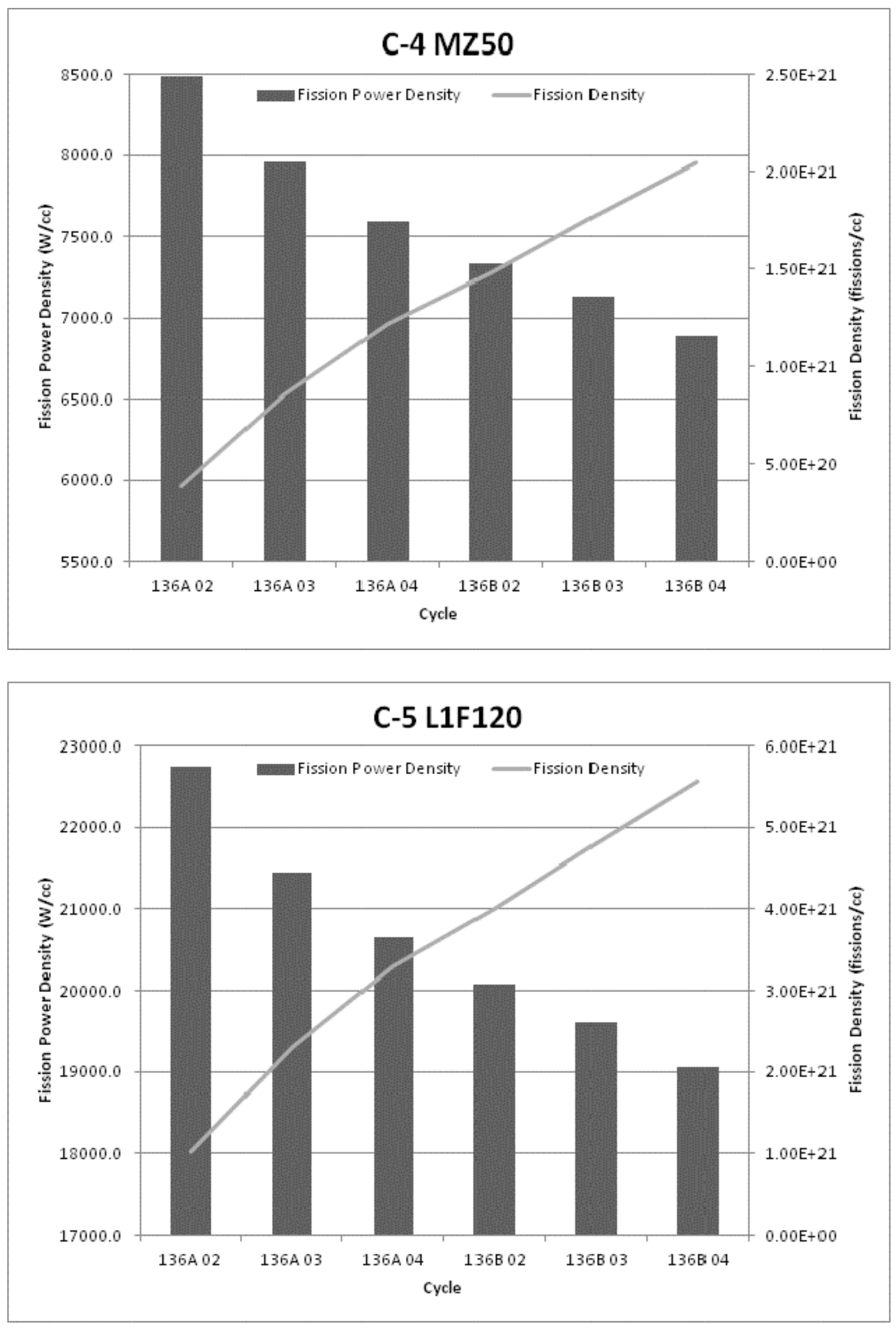

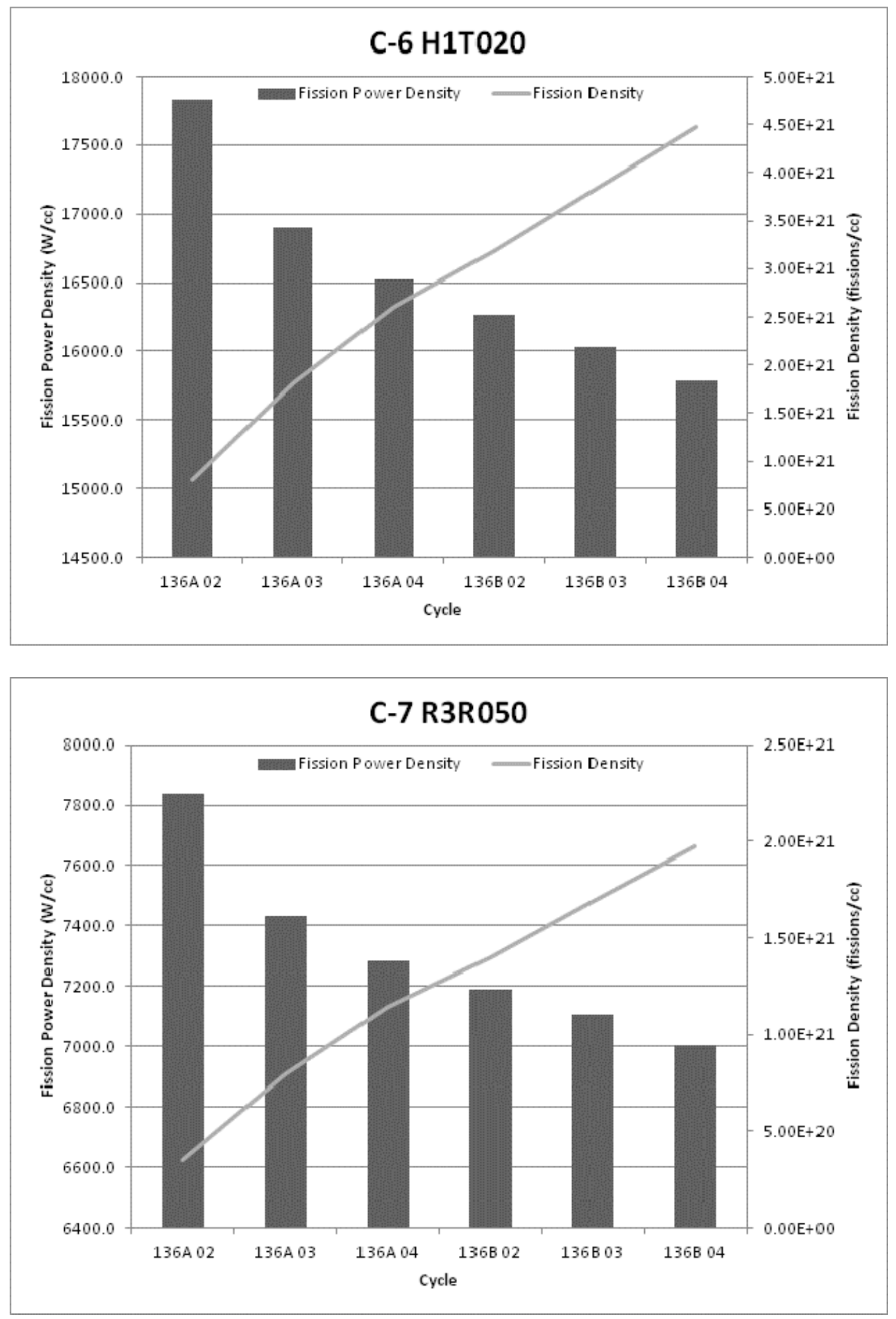

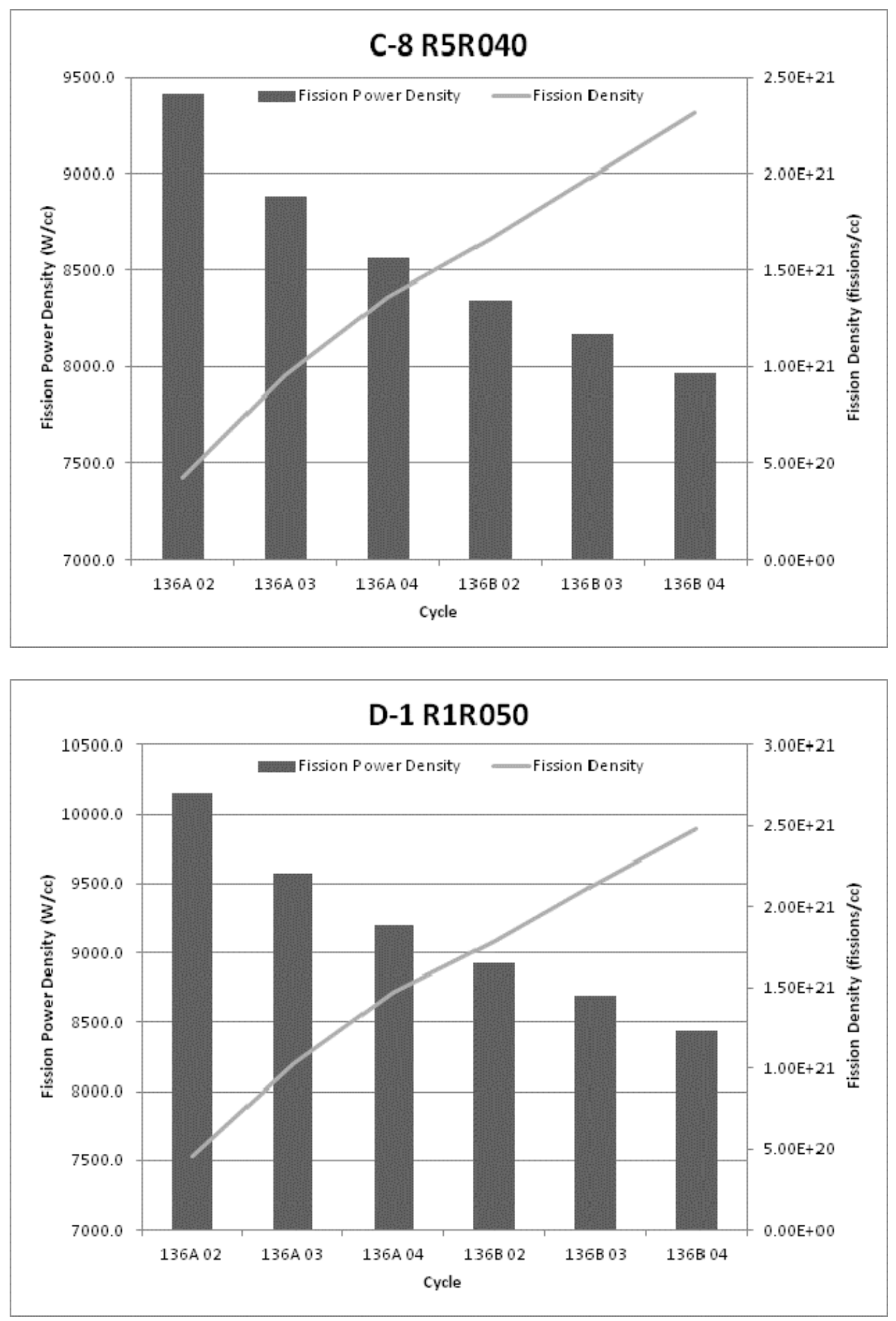

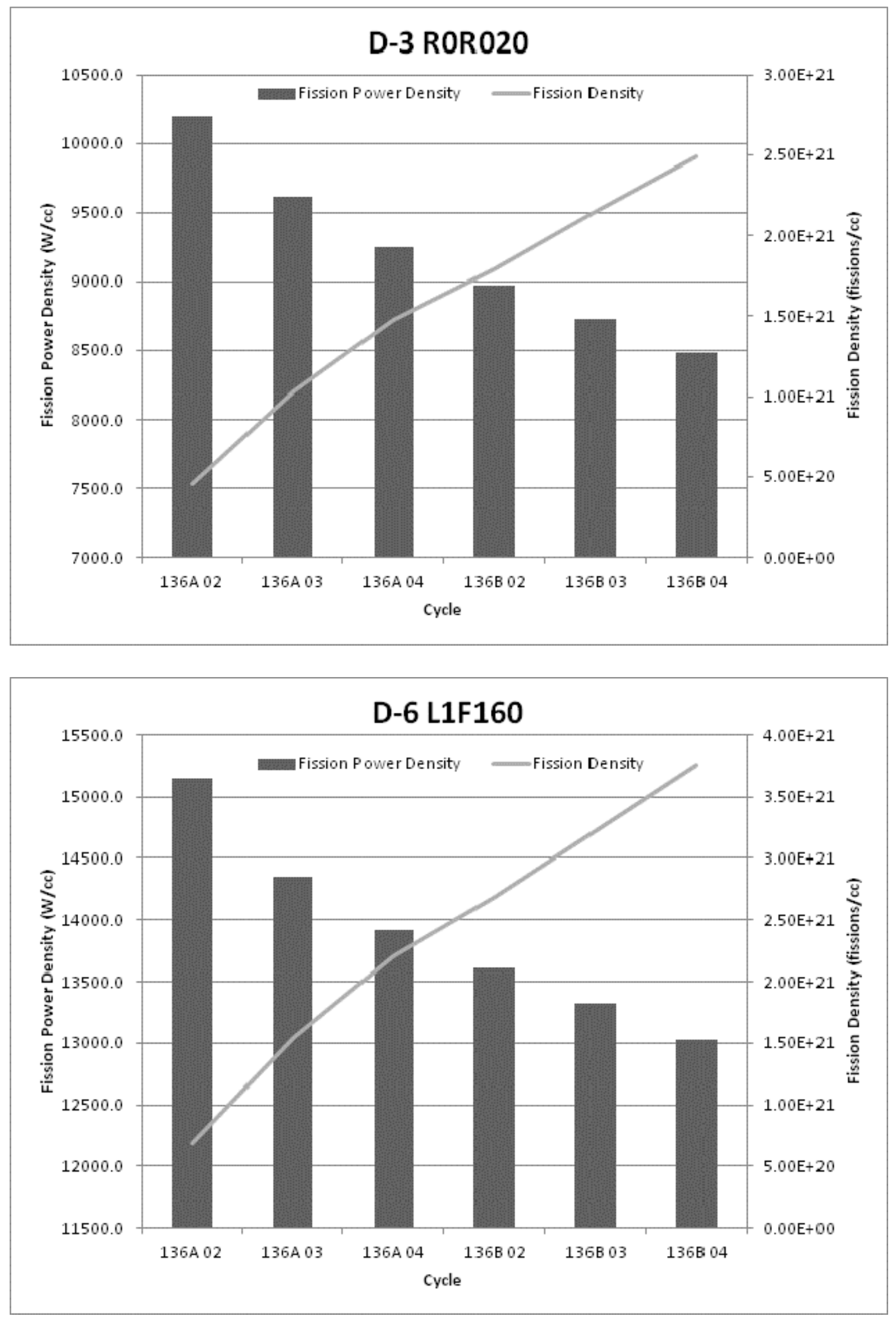

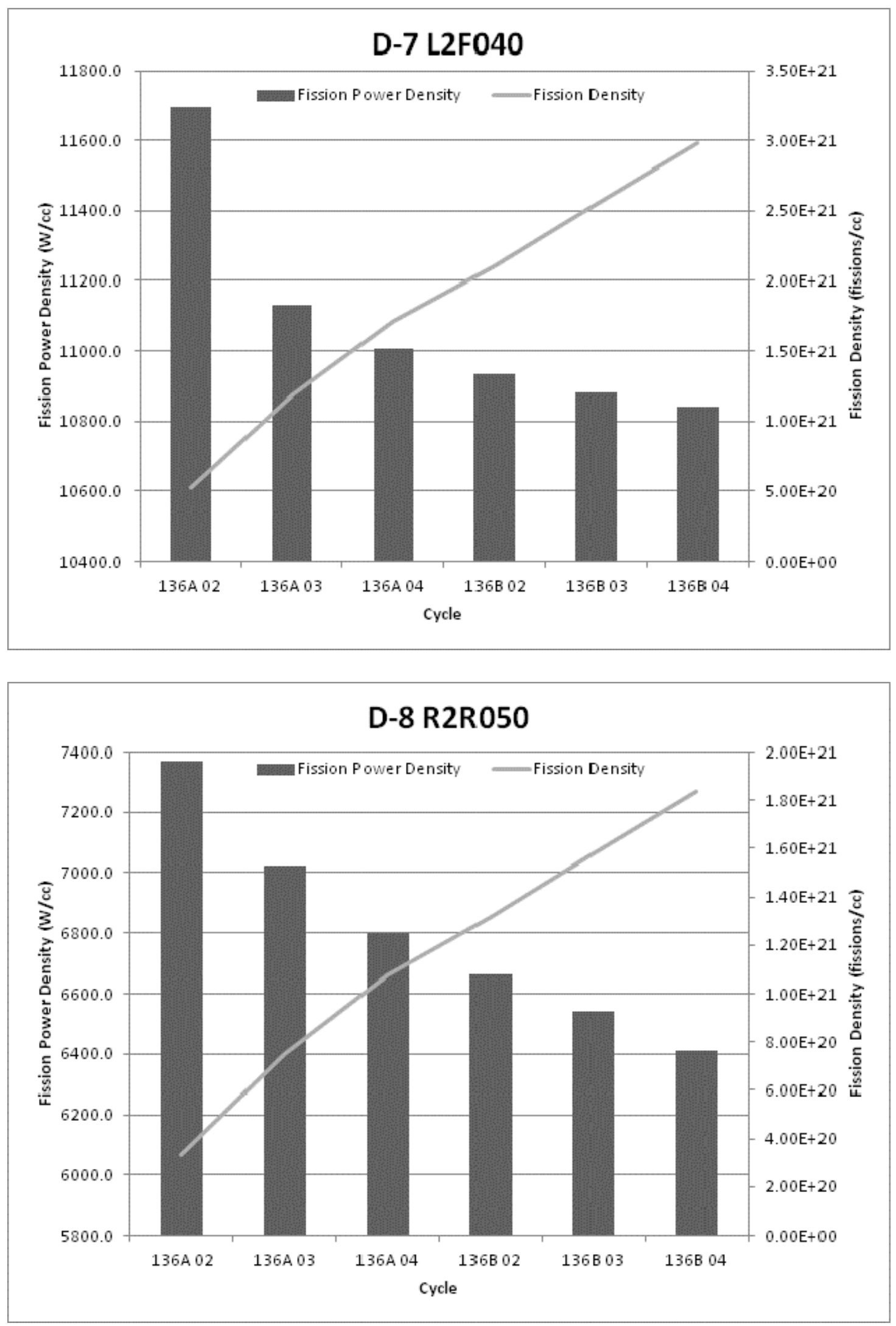


\section{A-2. RERTR-7B}
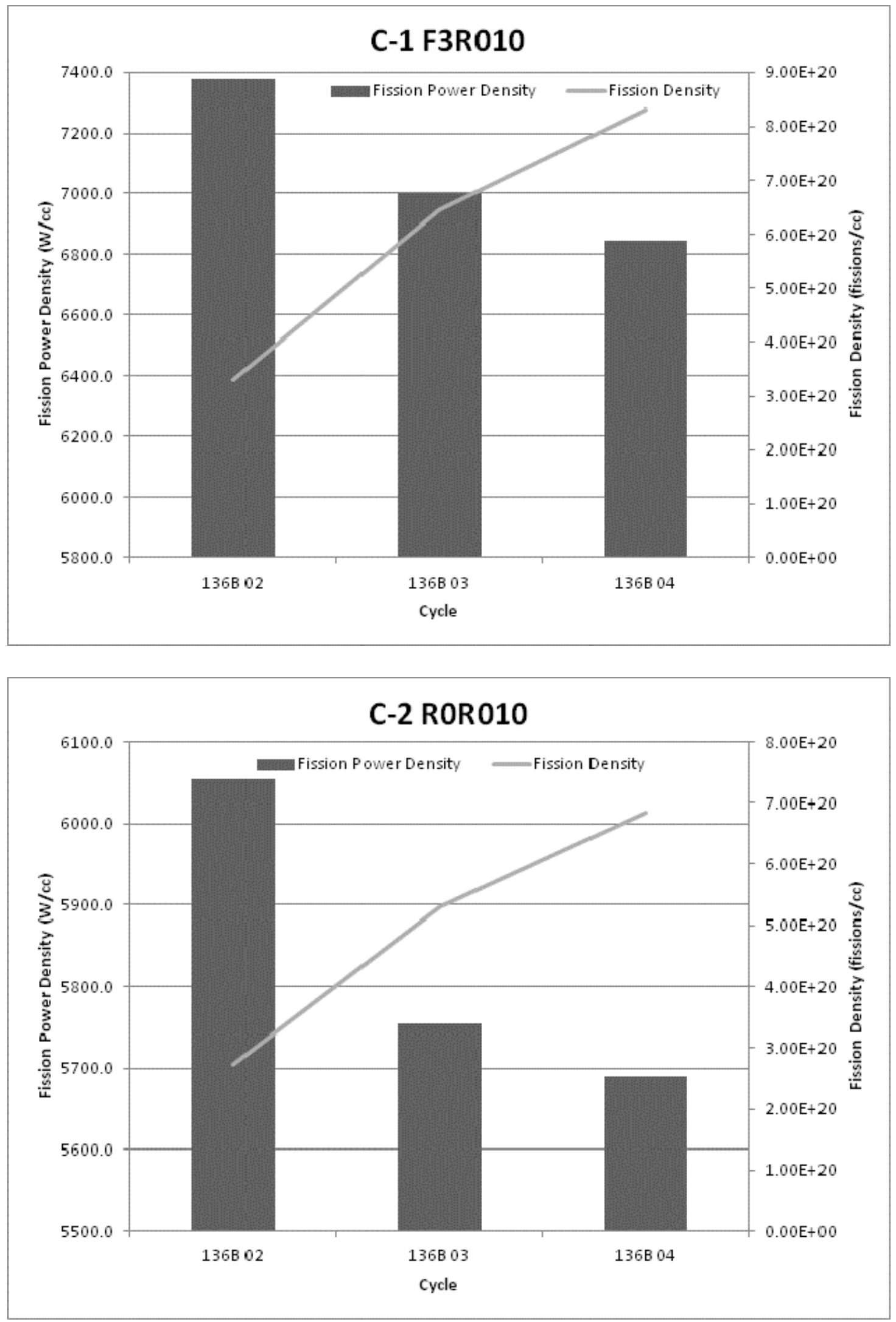

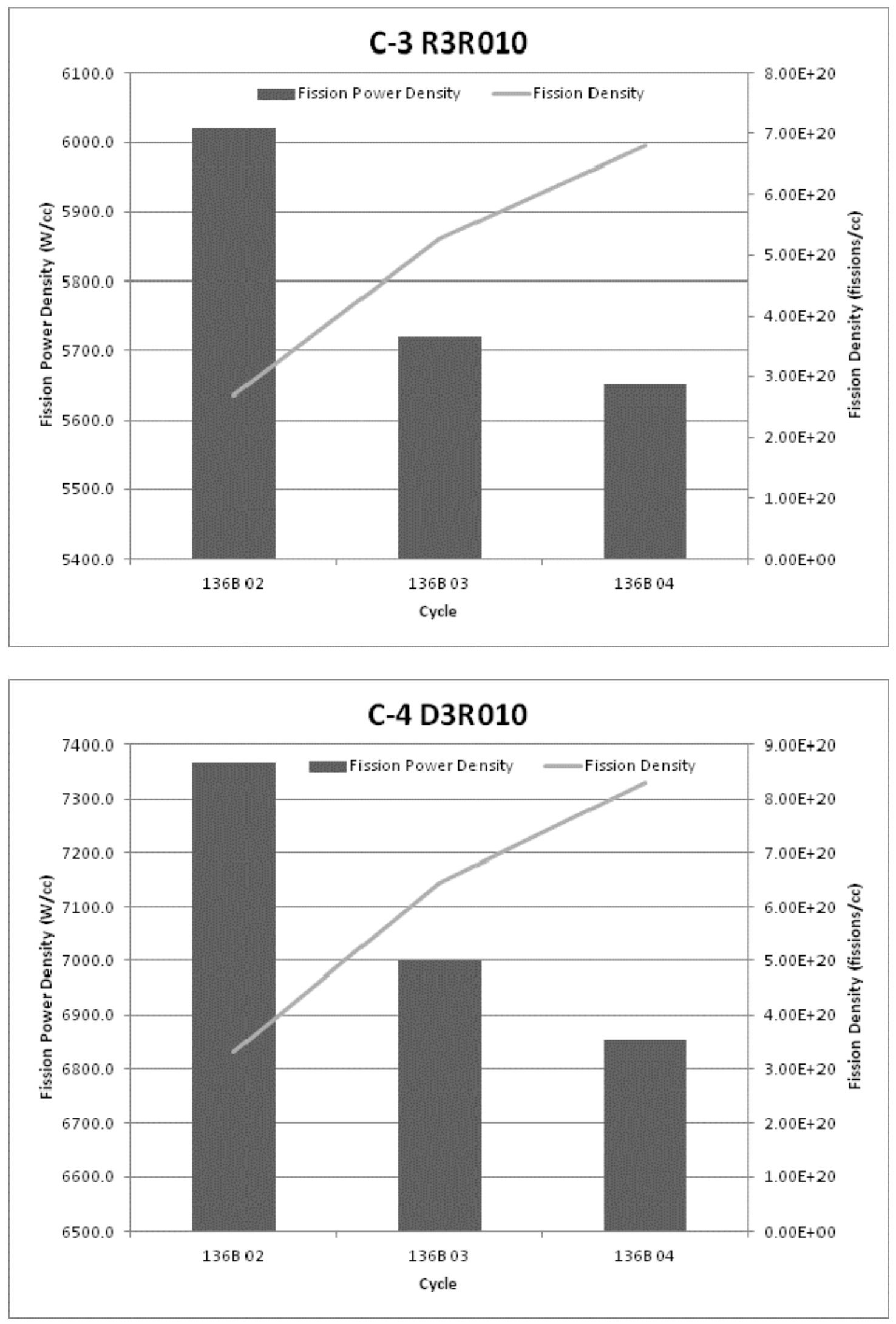
Appendix B

\section{Lillo Letter to Finley}




\section{INTEROFFICE MEMORANDUM}

Date: January 9, 2007

To: M. R. Finlay

MS 6188

$3-7572$

From: M. A. Lillo

MS 3885

6-5843

Subject: MCNP-Calculated Gradients Across RERTR-6 and RERTR-7A Miniplates Irradiated in ATR.

\subsubsection{References}

11. M. R. Finlay email communication to G. S. Chang, "Subject: power gradient across RERTR plates", December 19, 2006 (Attachment 1).

12. D. M. Wachs email communication to G. S. Chang and M. A. Lillo, attachment "RERTR-6 matrix asbuilt.xls", March 27, 2006.

13. D. M. Wachs intra-laboratory memorandum to Distribution, DMW-002-006, "As-Built Constituent Masses for RERTR-7A Mini-plate Experiment", March 6, 2006.

\subsubsection{Discussion}

As requested, calculations were performed to characterize the power gradient across each RERTR-6 and RERTR-7A miniplate. This memo reports the MCNP-calculated gradients for both the RERTR- 6 and RERTR-7A miniplates irradiated in ATR. Figures 1 and 2 were generated by the MCNP models used to perform RERTR-6 and RERTR-7A calculations, respectively. The thermal neutron flux local-to-average ratios (L2ARs) and fission rate L2ARs were calculated for the RERTR-6 miniplates irradiated in the ATR B-12 position and the RERTR-7A miniplates irradiated in the ATR B-11 position.

\subsubsection{Results}

The calculated thermal neutron flux L2AR and fission rate L2AR for RERTR-6 are tabulated in Tables 1 and 2, respectively. The calculated thermal neutron flux L2AR and fission rate L2AR for RERTR-7A is tabulated in Tables 3 and 4, respectively. Figures 3 through 6 were included to illustrate the data trends from the plate edge located nearest the core center (Node 1) to the plate edge located farthest from the core center (Node 20).

cc: D. M. Wachs, MS 6188

G. S. Chang, MS 3750

M. A. Lillo letter file (MAL-01-07) 

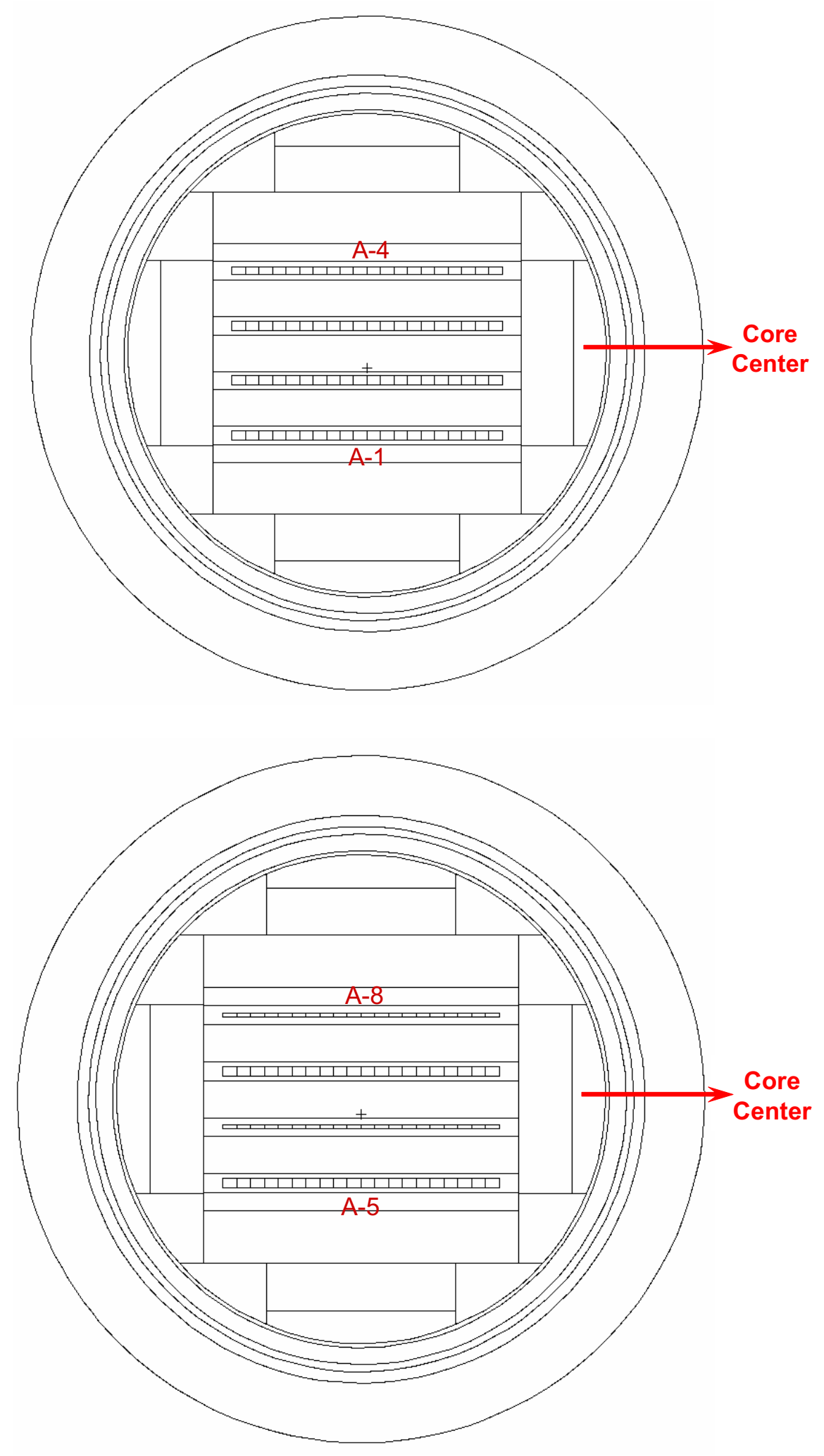

Figure 17 - RERTR-6 capsule A miniplates A-1 thru A-8 irradiated in ATR B-12 position. 

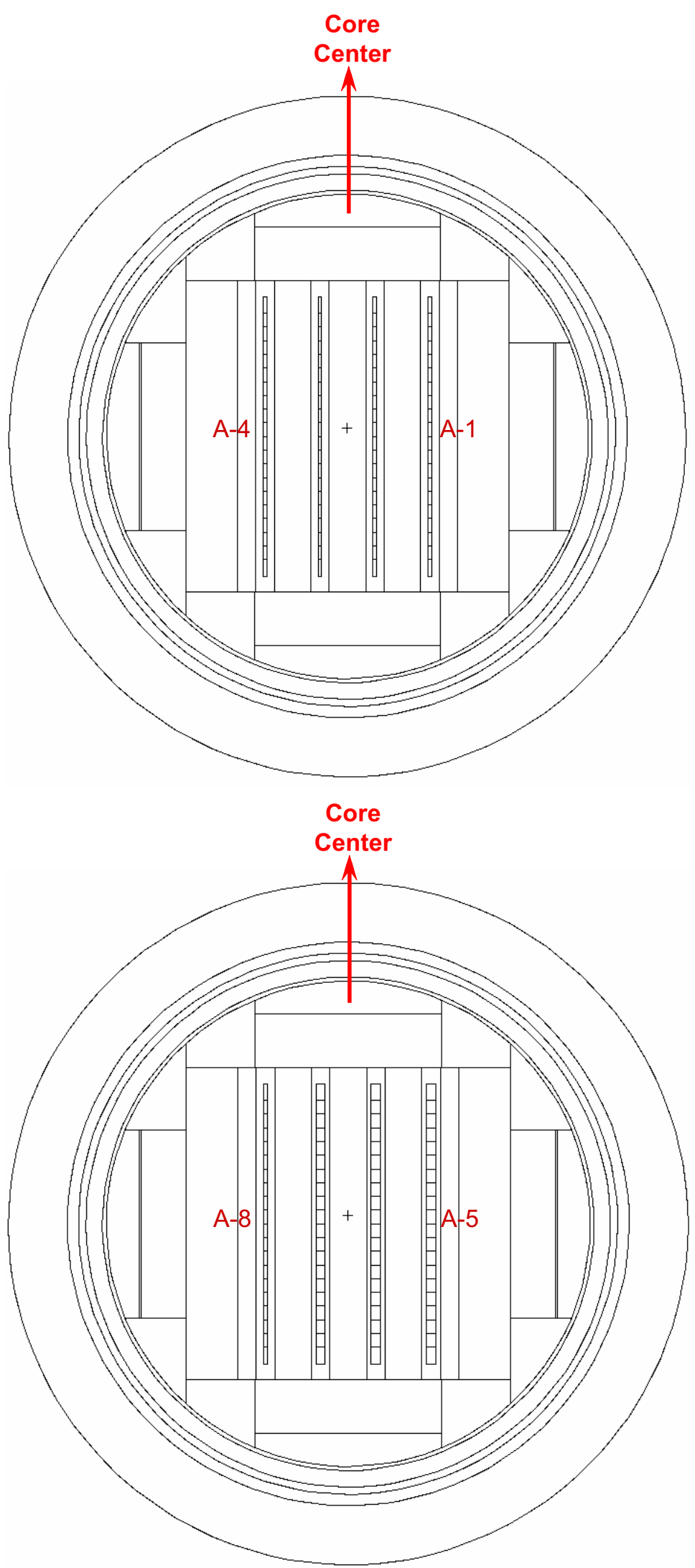

Figure 18 - RERTR-7A capsule A miniplates A-1 thru A-8 irradiated in ATR B-11 position. 
Table 1 - RERTR-6 thermal neutron flux local to average ratio (L2AR) along the plate width (from core center plate edge to outer plate edge)

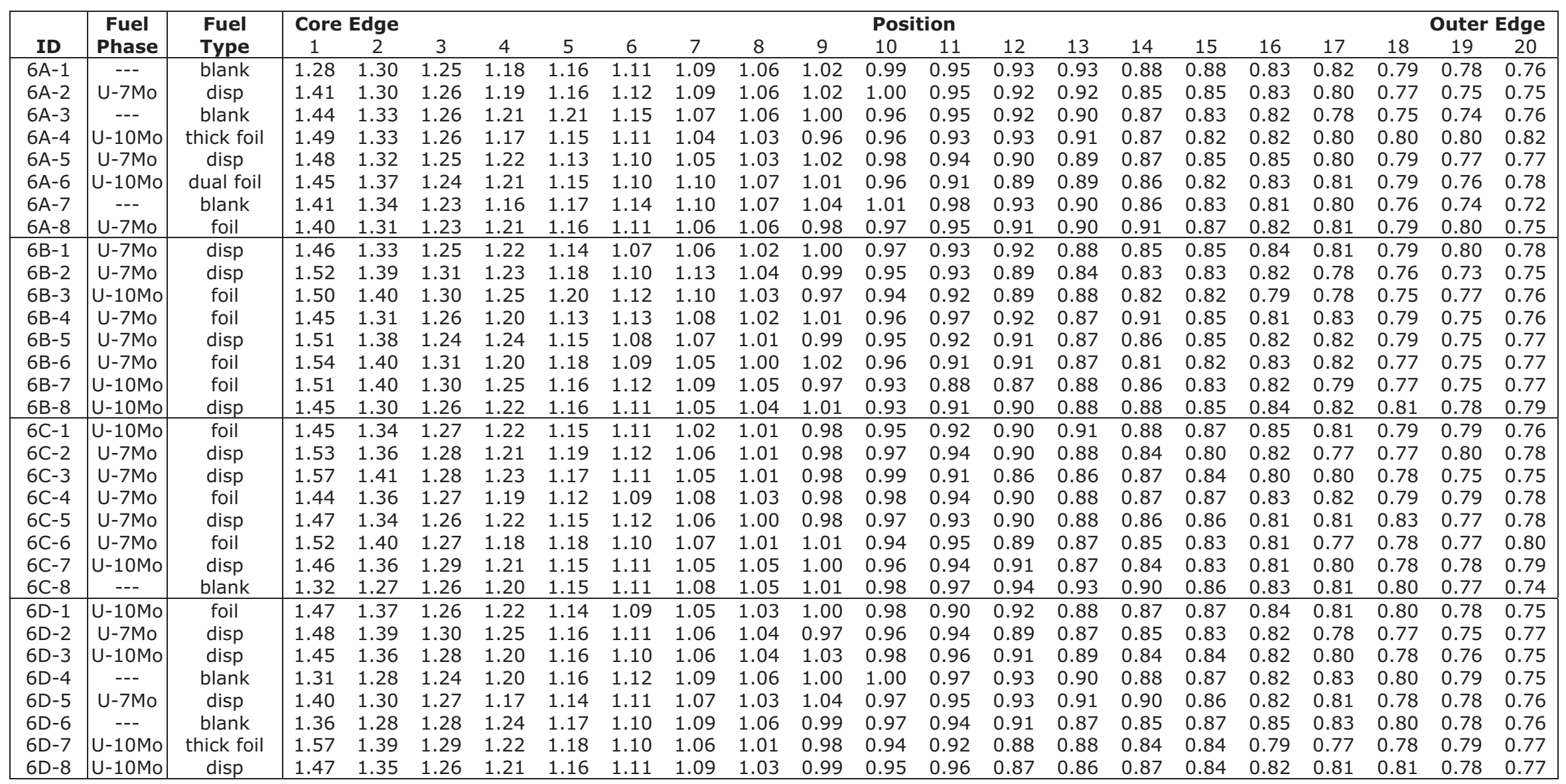


Table 2 - RERTR-6 fission rate tally local to average ratio (L2AR) along the plate width (from core center plate edge toward outer plate edge).

\begin{tabular}{|c|c|c|c|c|c|c|c|c|c|c|c|c|c|c|c|c|c|c|c|c|c|c|}
\hline \multirow[b]{2}{*}{ ID } & \multirow{2}{*}{$\begin{array}{c}\text { Fuel } \\
\text { Phase }\end{array}$} & \multirow{2}{*}{$\begin{array}{l}\text { Fuel } \\
\text { Type }\end{array}$} & \multicolumn{9}{|c|}{ Core Edge } & \multicolumn{8}{|c|}{ Position } & \multicolumn{3}{|c|}{ Outer Edge } \\
\hline & & & 1 & 2 & 3 & 4 & 5 & 6 & 7 & 8 & 9 & 10 & 11 & 12 & 13 & 14 & 15 & 16 & 17 & 18 & 19 & 20 \\
\hline $6 A-1$ & --- & blank & --- & --- & --- & --- & --- & --- & --- & --- & --- & --- & --- & --- & --- & --- & --- & --- & --- & --- & --- & --- \\
\hline $6 A-2$ & U-7Mo & disp & 1.41 & 1.31 & 1.26 & 1.19 & 1.16 & 1.12 & 1.08 & 1.06 & 1.02 & 1.00 & 0.95 & 0.92 & 0.92 & 0.85 & 0.85 & 0.83 & 0.80 & 0.76 & 0.75 & 0.75 \\
\hline $6 A-3$ & --- & blank & -- & --- & --- & --- & --- & --- & --- & --- & --- & --- & --- & --- & --- & --- & --- & --- & --- & --- & --- & --- \\
\hline $6 A-4$ & U-10Mo & thick foil & 1.49 & 1.33 & 1.26 & .18 & 1.15 & 1.11 & 1.04 & 1.03 & 0.96 & 0.96 & 0.93 & 0.93 & 0.91 & 0.87 & 0.82 & 0.82 & 0.80 & 0.80 & 0.80 & 0.82 \\
\hline $6 A-5$ & U-7Mo & disp & 1.49 & 1.32 & 1.24 & 1.22 & 1.13 & 1.10 & 1.05 & 1.03 & 1.02 & 0.98 & 0.94 & 0.90 & 0.89 & 0.87 & 0.85 & 0.85 & 0.80 & 0.79 & 0.76 & 0.77 \\
\hline $6 A-6$ & U-10Mo & dual foil & 1.43 & 1.35 & 1.22 & 1.20 & 1.14 & 1.09 & 1.09 & 1.05 & 0.99 & 0.94 & 0.89 & 0.88 & 0.87 & 0.84 & 0.82 & 0.83 & 0.81 & 0.79 & 0.76 & 0.78 \\
\hline $6 A-7$ & --- & blank & --- & --- & --- & --- & --- & --- & --- & --- & --- & --- & --- & --- & --- & --- & --- & --- & --- & --- & --- & --- \\
\hline $6 A-8$ & U-7Mo & & .40 & 1.31 & 1.23 & 1.21 & 1.16 & 1.11 & 1.05 & 1.06 & 0.98 & 0.97 & 0.95 & 0.91 & 0.90 & 0.91 & 0.87 & 0.82 & 0.81 & 0.79 & 0.80 & 0.75 \\
\hline $6 \mathrm{~B}-1$ & U-7Mo & disp & 47 & 1.33 & 1.25 & 1.22 & 1.14 & 1.07 & 1.06 & 1.03 & 1.00 & 0.97 & 0.93 & 0.92 & 0.88 & 0.85 & 0.85 & 0.84 & 0.82 & 0.80 & 0.80 & 0.78 \\
\hline $6 \mathrm{~B}-2$ & U-7Mo & disp & 53 & 1.39 & 1.31 & 1.23 & 1.18 & 1.10 & 1.13 & 1.04 & 1.00 & 0.95 & 0.92 & 0.89 & 0.84 & 0.83 & 0.83 & 0.82 & 0.78 & 0.76 & 0.73 & 0.75 \\
\hline $6 B-3$ & U-10Mo & foil & 1.51 & 1.40 & 1.30 & 1.25 & 1.20 & 1.12 & 1.10 & 1.03 & 0.97 & 0.94 & 0.92 & 0.89 & 0.89 & 0.82 & 0.82 & 0.79 & 0.78 & 0.75 & 0.77 & 0.76 \\
\hline $6 B-4$ & U-7Mo & il & 45 & 1.31 & 1.26 & 1.20 & 1.13 & 1.12 & 1.08 & 1.02 & 1.01 & 0.96 & 0.97 & 0.92 & 0.87 & 0.91 & 0.85 & 0.81 & 0.83 & 0.79 & 0.75 & 0.76 \\
\hline $6 B-5$ & U-7Mo & sp & L & 1.38 & 1.24 & 1.24 & 1.15 & 1.08 & 8 & 1.01 & 0.99 & 5 & 4 & 0. & 0.87 & 7 & 0.85 & 0.82 & 2 & 0.79 & 5 & 0.77 \\
\hline $6 B-6$ & U-7Mo & foil & 1 & 1.40 & 1.31 & 1.2 & 1.1 & 1.09 & 1.0 & .0 & 1.02 & 0.9 & 0 & 0. & $0 . \varepsilon$ & $0 . \varepsilon$ & 0.82 & 0. & 0.82 & 0.77 & 0.75 & 0.77 \\
\hline $6 \mathrm{~B}-7$ & U-10Mo & bil & 51 & 1.40 & 1.30 & 1.25 & 1.16 & 1.12 & 1.09 & 1.06 & 0.97 & 0.93 & 0.8 & 0.8 & 0.88 & 0.85 & 0.83 & 0.8 & 0.79 & 0.77 & 0.75 & 0.77 \\
\hline $6 B-8$ & U-10Mo & disp & 45 & 1.30 & 1.26 & 1.22 & 1.16 & 1.10 & 1.05 & 1.04 & 1.01 & 0.9 & 0.9 & 0.9 & 0.88 & 0.88 & 0.85 & 0.8 & 0.82 & 0.81 & 0.78 & 0.79 \\
\hline $6 C-1$ & U-10Mo & foil & 1.45 & 1.35 & 1.28 & 1.21 & 1.1 & 1.11 & 1.0 & .0 & 0.9 & 0.95 & 0.9 & 0.9 & 0.9 & 0.8 & .8 & $0 . \varepsilon$ & $0 . \varepsilon$ & .79 & 0.79 & 0.76 \\
\hline $6 C-2$ & U-7Mo & disp & 53 & 1.36 & 1.28 & 1.21 & 1.19 & 1.12 & 1.06 & 1.01 & 0.98 & 0.97 & 0.9 & 0.9 & 0.87 & 0.83 & 0.80 & 0.8 & 0.78 & 0.77 & 0.81 & 0.78 \\
\hline $6 C-3$ & U-7Mo & disp & 1.57 & 1.41 & 1.28 & 1.22 & 1.16 & 1.10 & 1.05 & 1.01 & 0.98 & 0.99 & 0.91 & 0.86 & 0.86 & 0.87 & 0.84 & 0.80 & 0.80 & 0.79 & 0.75 & 0.76 \\
\hline $6 C-4$ & U-7Mo & bil & 1.44 & 1.36 & 1.27 & 1.19 & 1.12 & 1.09 & 1.08 & 1.03 & 0.98 & 0.98 & 0.9 & 0.9 & 0.89 & 0.87 & 0.87 & 0.83 & 0.82 & 0.79 & 0.79 & 0.78 \\
\hline $6 C-5$ & U-7Mo & disp & 1.48 & 1.35 & 1.26 & 1.22 & 1.15 & 1.12 & 1.06 & 1.00 & 0.99 & 0.97 & 0.93 & 0.9 & 0.88 & 0.86 & 0.86 & 0.81 & 0.81 & 0.82 & 0.77 & 0.78 \\
\hline $6 C-6$ & U-7Mo & foil & 1.52 & 1.40 & 1.27 & 1.17 & 1.18 & 1.09 & 1.07 & 1.01 & 1.01 & 0.95 & 0.9 & 0.90 & 0.87 & 0.85 & 0.83 & 0.81 & 0.77 & 0.78 & 0.77 & 0.81 \\
\hline $6 C-7$ & U-10Mo & $\mathrm{sp}$ & 1.46 & 1.35 & 1.29 & 1.21 & 1.16 & 1.11 & 1.05 & 1.04 & 0.99 & 0.96 & 0.94 & 0.91 & 0.87 & 0.84 & 0.83 & 0.81 & 0.80 & 0.78 & 0.78 & 0.79 \\
\hline $6 C-8$ & -- & ank & --- & --- & --- & --- & --- & --- & --- & --- & --- & --- & --- & --- & --- & --- & --- & -- & -- & --- & --- & --- \\
\hline $6 \mathrm{D}-1$ & U-10Mo & ii & 47 & 1.37 & 1.26 & 1.22 & 1.1 & 1.09 & .04 & 1.02 & 0.9 & 000 & 0.9 & 0.9 & 0.8 & 0.8 & 0.8 & $0 . \varepsilon$ & 0.82 & 0.80 & 0.78 & 0.75 \\
\hline $6 D-2$ & U-7Mo & $s p$ & 1 & 1.3 & 1.31 & .25 & 1.1 & 1.11 & .06 & 1.0 & 0.9 & 6 & 0.9 & 0.8 & 0.87 & 0.85 & 0.83 & 0.8 & 0.78 & 0.77 & 0.75 & 0.77 \\
\hline $6 D-3$ & U-10Mo & & 1.45 & 1.36 & 1.27 & 1.20 & 1.16 & 1.10 & 1.06 & 1.04 & 1.03 & 0.98 & 0.96 & 0.9 & 0.89 & 0.84 & 0.85 & 0.82 & 0.80 & 0.78 & 0.76 & 0.75 \\
\hline $6 D-4$ & --- & blank & --- & --- & --- & --- & --- & --- & --- & --- & --- & --- & --- & --- & --- & --- & --- & --- & --- & --- & --- & --- \\
\hline $6 D-5$ & U-7Mo & & 1.41 & 1.30 & 1.27 & 1.17 & 1.14 & 1.10 & 1.07 & 1.03 & 1.04 & 0.97 & 0.9 & 0.93 & 0.90 & 0.90 & 0.86 & 0.82 & 0.81 & 0.78 & 0.78 & 0.76 \\
\hline $6 D-6$ & --- & ank & --- & --- & --- & --- & --- & --- & --- & --- & --- & --- & --- & --- & --- & --- & --- & --- & --- & --- & --- & --- \\
\hline $6 D-7$ & U-10Mo & thick foil & 1.57 & 1.39 & 1.29 & 1.22 & 1.18 & 1.10 & 1.06 & 1.01 & 0.98 & 0.94 & 0.92 & 0.8 & 0.88 & 0.84 & 0.84 & 0.79 & 0.77 & 0.78 & 0.79 & 0.77 \\
\hline $6 D-8$ & U-10Mo & disp & 1.48 & 1.36 & 1.27 & 1.21 & 1.16 & 1.10 & 1.09 & 1.03 & 0.99 & 0.95 & 0.96 & 0.87 & 0.86 & 0.87 & 0.84 & 0.82 & 0.81 & 0.81 & 0.78 & 0.77 \\
\hline
\end{tabular}


Table 3 - RERTR-7A thermal neutron flux local to average ratio (L2AR) along the plate width (from core center plate edge toward outer plate edge)

\begin{tabular}{|c|c|c|c|c|c|c|c|c|c|c|c|c|c|c|c|c|c|c|c|c|c|c|}
\hline \multirow[b]{2}{*}{ ID } & \multirow{2}{*}{$\begin{array}{c}\text { Fuel } \\
\text { Phase }\end{array}$} & \multirow{2}{*}{$\begin{array}{l}\text { Fuel } \\
\text { Type }\end{array}$} & \multicolumn{9}{|c|}{ Core Edge } & \multicolumn{9}{|c|}{ Position } & \multicolumn{2}{|c|}{ Outer Edge } \\
\hline & & & 1 & 2 & 3 & 4 & 5 & 6 & 7 & 8 & 9 & 10 & 11 & 12 & 13 & 14 & 15 & 16 & 17 & 18 & 19 & 20 \\
\hline $7 \mathrm{~A}-1$ & -1 & blank & 1.25 & 1.22 & 1.19 & 1.17 & 1.15 & 1.12 & 1.09 & 1.07 & 1.06 & 1.03 & 0.99 & 0.96 & 0.92 & 0.92 & 0.90 & 0.84 & 0.82 & 0.80 & 0.77 & 0.74 \\
\hline $7 A-2$ & --- & blank & 1.27 & 1.23 & 1.22 & .17 & .15 & .13 & 1.09 & 1.08 & 1.05 & 1.02 & 0.99 & 0.95 & 0.94 & 0.90 & 0.87 & .86 & .80 & .79 & .76 & 0.73 \\
\hline $7 A-3$ & --- & blank & 1.27 & 1.24 & 1.21 & 1.20 & 1.15 & 1.13 & 1.13 & 1.06 & 1.03 & 1.01 & 0.98 & 0.97 & 0.93 & 0.91 & 0.88 & 0.84 & .82 & .79 & .75 & 0.70 \\
\hline $7 A-4$ & --- & blank & 1.24 & 1.22 & 1.20 & 1.16 & 1.14 & 1.10 & 1.08 & 1.06 & 1.05 & 1.03 & 0.98 & 0.97 & 0.96 & 0.94 & 0.90 & 0.87 & 0.82 & 0.79 & 0.76 & 0.73 \\
\hline $7 A-5$ & U-7Mo & disp & 1.74 & 1.48 & 1.35 & 1.21 & 1.16 & 1.10 & 1.03 & 0.98 & 0.96 & 0.93 & 0.88 & 0.85 & 0.83 & 0.81 & 0.80 & 0.79 & 0.76 & 0.76 & 0.77 & 0.81 \\
\hline $7 A-6$ & U-10Mo & disp & 2.05 & 1.65 & 1.45 & 1.28 & 1.19 & 1.08 & 1.02 & 0.94 & 0.89 & 0.85 & 0.82 & 0.79 & 0.77 & 0.75 & 0.73 & 0.73 & 0.74 & 0.72 & 0.75 & 0.79 \\
\hline $7 A-7$ & U-7Mo & disp & 2.01 & 1.61 & 1.41 & 1.26 & 1.20 & 1.10 & 0.99 & 0.94 & 0.89 & 0.87 & 0.84 & 0.82 & 0.79 & 0.76 & 0.74 & 0.73 & 0.73 & 0.74 & .74 & 0.82 \\
\hline $7 A-8$ & $\mathrm{U}-12 \mathrm{Mo}$ & il & 1 & .44 & 1.34 & 1.24 & 1.17 & 1.11 & 1.06 & 1.01 & 0.97 & 0.93 & 0.91 & 0.87 & 0.83 & 0.83 & 0.82 & 0.79 & 0.77 & 0.77 & .77 & 0.76 \\
\hline 7B-1 & U-7Mo & disp & 1 & 1.46 & 1.34 & 1.24 & 1.15 & 1.09 & 1.02 & 0.98 & 0.95 & 0. & 0 & 0.86 & 0.83 & 0.82 & 31 & .79 & .76 & 0.76 & 76 & 0.80 \\
\hline 7B-2 & U-7Mo & sp & 2.03 & 1.63 & 1.44 & 1.31 & 1.19 & 1.10 & 1.02 & 0.94 & 0.89 & 0 & 1 & 79 & 0.77 & 4 & 74 & 73 & .74 & .73 & 75 & 0.80 \\
\hline 7B-3 & U-7Mo & disp & 2 & 1.63 & 1.46 & 1. & 19 & 1.09 & 1. & 0 & 0 & 0 & 3 & 1 & 7 & 0 & 4 & 2 & .73 & .72 & 4 & .81 \\
\hline 7B-4 & $12 \mathrm{Mo}$ & foil & 1 & 1.43 & 1.37 & 1.26 & 1.16 & 1.07 & 1.06 & 1. & 0.96 & 0 & 0. & 8 & 0 & 0. & 0 & 8 & 76 & .76 & 77 & .79 \\
\hline 7B-5 & $10 \mathrm{Mo}$ & foil & 1.64 & 1.42 & 1.34 & 1.24 & 1.17 & 1.10 & 1.04 & 1.00 & 0.96 & 0. & 0.92 & 0.87 & 0.84 & 0.82 & 0.80 & 0.79 & .78 & 0.77 & 78 & 0.77 \\
\hline 7B-6 & $-10 \mathrm{Mo}$ & disp & 1.92 & 1.57 & 1.43 & 1.27 & 1.17 & 1.10 & 1.02 & 0.96 & 0.92 & 0.87 & 0.86 & 0.81 & 0.79 & 0.77 & 0.75 & 0.73 & 0.73 & 0.74 & .76 & 0.81 \\
\hline 7B-7 & U-10Mo & foil & 1.80 & 1.54 & 1.38 & 1.30 & 1.19 & 1.09 & 1.06 & 1.00 & 0.95 & 0.92 & 0.87 & 0.83 & 0.82 & 0.79 & 0.79 & 0.77 & 0.75 & 0.74 & .75 & 0.66 \\
\hline $7 B-8$ & U-7Mo & foil & 1.56 & 1.42 & 1.31 & 1.24 & 1.19 & 1.10 & 1.05 & 1.01 & 0.98 & 0.96 & 0.92 & 0.87 & 0.86 & 0.84 & 0.82 & 0.80 & 0.79 & 0.76 & .76 & 0.76 \\
\hline 7C-1 & $-12 \mathrm{Mo}$ & foil & 1.65 & 1.41 & 1.33 & 1.24 & 1.16 & 1.08 & 1.05 & 1.03 & 0.97 & 0.9 & 0.91 & 0.88 & 0.84 & 0.82 & 0.81 & 0.80 & 0.77 & 0.77 & .77 & 0.78 \\
\hline $7 C-2$ & U-10Mo & foil & 1.90 & 1.61 & 1.42 & 1.29 & 1.19 & 1.10 & 1.04 & 0.98 & 0.91 & 0.87 & 0.82 & 0.82 & 0.79 & 0.75 & 0.75 & 0.74 & 0.73 & 0.74 & .76 & 0.79 \\
\hline $7 C-3$ & U-10Mo & il & 1.82 & 1.52 & 1.40 & 1.30 & 1.21 & 1.11 & 1.05 & 0.97 & 0.92 & 0 & 0.88 & 0.83 & 0.78 & 0. & 0.77 & 0 & 4 & 74 & 4 & 0.78 \\
\hline 7C-4 & Mo & il & 1 & 1.46 & 1.34 & 1.25 & 1.15 & 1.11 & 1.06 & 0.99 & 0.94 & & 89 & 0.87 & 0.84 & 34 & 0.80 & 80 & .78 & .76 & 76 & 0.78 \\
\hline $7 C-5$ & U-10Mo & il & 1 & 1.45 & 1.32 & 1.24 & 1.16 & 1.10 & 1.05 & 99 & 7 & 1 & 0 & 36 & 35 & 3 & 32 & 80 & .79 & 77 & 77 & 0.77 \\
\hline 7C-6 & $\mathrm{U}-12 \mathrm{Mo}$ & foil & 1 & 1.60 & 1.44 & 1.32 & 1.17 & 1.09 & 1.03 & 0 & 1 & 0.88 & 4 & 2 & 0 & 0. & 0 & 4 & .74 & .72 & .73 & 0.78 \\
\hline 7C-7 & U-7Mo & disp & 1.99 & 1.62 & 1.43 & 1.31 & 1.18 & 1.09 & 1.02 & 0.98 & 0.91 & 0.87 & 0.81 & 0.79 & 0.78 & 0.76 & 0.72 & 0.71 & 0.73 & 0.72 & 0.76 & 0.82 \\
\hline $7 C-8$ & U-7Mo & disp & 1.75 & 1.47 & 1.33 & 1.24 & 1.14 & 1.07 & 1.04 & 0.99 & 0.95 & 0.92 & 0.91 & 0.88 & 0.84 & 0.82 & 0.80 & 0.78 & 0.79 & 0.76 & 0.74 & 0.79 \\
\hline 7D-1 & U-7Mo & disp & 1.64 & 1.40 & 1.28 & 1.19 & 1.15 & 1.07 & 1.06 & 1.03 & 1.01 & 0.95 & 0.93 & 0.88 & 0.86 & 0.83 & 0.83 & 0.81 & 0.78 & 0.77 & 0.76 & 0.78 \\
\hline 7D-2 & --- & blank & 1.55 & 1.43 & 1.35 & 1.24 & 1.22 & 1.12 & 1.08 & 1.03 & 1.00 & 0.95 & 0.92 & 0.88 & 0.85 & 0.83 & 0.81 & 0.78 & 0.76 & 0.74 & 0.74 & 0.72 \\
\hline 7D-3 & U-7Mo & disp & 1.65 & 1.41 & 1.31 & 1.23 & 1.17 & 1.11 & 1.06 & 1.01 & 1.00 & 0.94 & 0.89 & 0.90 & 0.87 & 0.84 & 0.81 & 0.78 & 0.78 & 0.76 & 0.72 & 0.75 \\
\hline 7D-4 & --- & blank & 1.41 & 1.34 & 1.29 & 1.22 & 1.15 & 1.11 & 1.09 & 1.05 & 1.02 & 0.98 & 0.94 & 0.92 & 0.90 & 0.87 & 0.85 & 0.81 & 0.78 & 0.77 & 0.74 & 0.74 \\
\hline 7D-5 & --- & blank & 1.43 & 1.38 & 1.30 & 1.21 & 1.18 & 1.09 & 1.08 & 1.05 & 1.03 & 0.96 & 0.96 & 0.94 & 0.88 & 0.86 & 0.84 & 0.80 & 0.78 & 0.75 & 0.75 & 0.73 \\
\hline 7D-6 & U-10Mo & foil & 1.75 & 1.50 & 1.37 & 1.27 & 1.17 & 1.09 & 1.06 & 1.00 & 0.98 & 0.92 & 0.88 & 0.87 & 0.83 & 0.80 & 0.79 & 0.78 & 0.75 & 0.71 & 0.72 & 0.76 \\
\hline 7D-7 & U-10Mo & foil & 1.95 & 1.57 & 1.41 & 1.27 & 1.15 & 1.06 & 1.02 & 0.96 & 0.92 & 0.88 & 0.84 & 0.81 & 0.80 & 0.80 & 0.75 & 0.73 & 0.74 & 0.74 & 0.76 & 0.82 \\
\hline 7D-8 & U-7Mo & disp & 1.80 & 1.49 & 1.34 & 1.25 & 1.13 & 1.06 & 1.02 & 0.98 & 0.97 & 0.91 & 0.88 & 0.85 & 0.85 & 0.81 & 0.79 & 0.78 & 0.76 & 0.76 & 0.77 & 0.81 \\
\hline
\end{tabular}


Table 4 - RERTR-7A fission rate tally local to average ratio (L2AR) along the plate width (from core center plate edge toward outer plate edge)

\begin{tabular}{|c|c|c|c|c|c|c|c|c|c|c|c|c|c|c|c|c|c|c|c|c|c|c|}
\hline \multirow[b]{2}{*}{ ID } & \multirow{2}{*}{$\begin{array}{c}\text { Fuel } \\
\text { Phase } \\
\end{array}$} & \multirow{2}{*}{$\begin{array}{l}\text { Fuel } \\
\text { Type }\end{array}$} & \multicolumn{9}{|c|}{ Core Edge } & \multicolumn{8}{|c|}{ Position } & \multicolumn{3}{|c|}{ Outer Edge } \\
\hline & & & 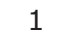 & 2 & 3 & 4 & 5 & 6 & 7 & 8 & 9 & 10 & 11 & 12 & 13 & 14 & 15 & 16 & 17 & 18 & 19 & 20 \\
\hline $7 A-1$ & --- & blank & --- & --- & --- & --- & --- & --- & --- & --- & --- & --- & --- & --- & --- & --- & --- & --- & --- & --- & --- & --- \\
\hline $7 A-2$ & --- & blank & --- & --- & --- & --- & --- & --- & --- & --- & --- & --- & --- & --- & --- & --- & --- & --- & --- & --- & --- & --- \\
\hline $7 A-3$ & --- & & --- & --- & --- & --- & --- & --- & --- & --- & --- & --- & --- & --- & --- & --- & --- & --- & --- & --- & --- & --- \\
\hline $7 A-4$ & --- & blank & --- & --- & --- & --- & --- & --- & --- & --- & --- & --- & --- & --- & --- & --- & --- & --- & --- & --- & --- & --- \\
\hline $7 A-5$ & U-7Mo & disp & 1.75 & 1.48 & 1.35 & 1.21 & 1.16 & 1.10 & 1.03 & 0.97 & 0.96 & 0.93 & 0.88 & 0.85 & 0.83 & 0.81 & 0.80 & 0.79 & 0.76 & 0.77 & 0.77 & 0.81 \\
\hline $7 A-6$ & $\mathrm{U}-10 \mathrm{Mo}$ & disp & .06 & 1.65 & 1.45 & 1.28 & 1.18 & 1.07 & 1.02 & 0.94 & 0.89 & 0.85 & 0.82 & 0.79 & 0.77 & 0.75 & 0.73 & 0.73 & 0.74 & 0.72 & 0.75 & 0.79 \\
\hline $7 A-7$ & U-7Mo & disp & .01 & 1.61 & 1.41 & 1.26 & 1.20 & 1.10 & 0.98 & 0.94 & 0.89 & 0.87 & 0.84 & 0.82 & 0.79 & 0.76 & 0.74 & 0.73 & 0.74 & 0.74 & 0.75 & 0.83 \\
\hline $7 A-8$ & $\mathrm{U}-12 \mathrm{Mo}$ & foil & .63 & 1.45 & 1.34 & 1.23 & 1.16 & 1.11 & 1.06 & 1.01 & 0.97 & 0.93 & 0.91 & 0.87 & 0.83 & 0.83 & 0.82 & 0.79 & 0.78 & 0.77 & 0.77 & 0.76 \\
\hline 7B-1 & U-7Mo & disp & .75 & 1.46 & 1.34 & 1.24 & 1.15 & 1.09 & 1.02 & 0.98 & 0.95 & 0.93 & 0.89 & 0.86 & 0.83 & 0.82 & 0.81 & 0.79 & 0.76 & 0.76 & 0.76 & 0.81 \\
\hline $7 B-2$ & U-7Mo & disp & .03 & 1.64 & 1.44 & 1.30 & 1.19 & 1.10 & 1.02 & 0.94 & 0.89 & 0.85 & 0.81 & 0.79 & 0.77 & 0.74 & 0.74 & 0.73 & 0.74 & 0.73 & 0.76 & 0.81 \\
\hline $7 B-3$ & U-7Mo & disp & .01 & 1.63 & 1.46 & 1.29 & 1.19 & 1.09 & 1.01 & 0.94 & 0.90 & 08 & 0.83 & 0.81 & 0.78 & 0.7 & 0.74 & 0.72 & 0.73 & 0.73 & 0.74 & 0.81 \\
\hline $7 B-4$ & $\mathrm{U}-12 \mathrm{Mo}$ & foil & 1.63 & 1.43 & 1.37 & 1.26 & 1.16 & 1.07 & 1.06 & 1.00 & 0.96 & 0 & 0.91 & 0.88 & 0.83 & 0.83 & 0.81 & 0.79 & 0.76 & 0.76 & 0.77 & 0.79 \\
\hline 7B-5 & $\mathrm{U}-10 \mathrm{Mo}$ & foil & 1.63 & 1.42 & 1.34 & 1.24 & 1.17 & 0 & 1.04 & 1.00 & 0.96 & ] & 0.91 & 87 & .84 & 0 & 80 & 0.79 & 0.78 & 0.77 & .78 & 0.77 \\
\hline $7 B-6$ & U-10Mo & disp & 3 & 1.57 & 1.43 & 1.27 & 1.17 & 1.1 & 1.02 & 0.96 & 0.92 & 0.87 & 0.86 & 0.81 & .7 & 0. & 0.75 & 0.74 & 0.73 & 0.74 & 0.76 & 0.82 \\
\hline 7B-7 & $\mathrm{U}-10 \mathrm{Mo}$ & foil & .80 & 1.53 & 1.38 & 1.30 & 1.18 & 1.08 & 1.05 & 0.99 & 0.94 & 0.9 & 0.86 & 0.82 & 0.81 & 0.7 & 0.79 & 0.77 & 0.74 & 0.74 & 0.74 & 0.76 \\
\hline $7 B-8$ & U-7Mo & foil & .56 & 1.42 & 1.31 & 1.24 & 1.19 & 1.10 & 1.04 & 1.01 & 0.98 & 0.9 & 0.91 & 0.88 & 0.86 & 0.84 & 0.82 & 0.80 & 0.79 & 0.76 & 0.76 & 0.76 \\
\hline 7C-1 & $12 \mathrm{Mo}$ & foil & 65 & 1.41 & 1.33 & 1.24 & 1.16 & 1.08 & 1.0 & 1.03 & 0.97 & 0.92 & 0.9 & 0.8 & .8 & 0.8 & 0.81 & 0.80 & .78 & 0.77 & 0.77 & 0.78 \\
\hline $7 C-2$ & $10 \mathrm{Mo}$ & foil & .90 & 1.61 & 1.42 & 1.29 & 1.19 & 1.10 & 1.04 & 0.98 & 0.91 & 0.8 & 0.82 & 0.82 & 0.80 & 0.7 & 0.75 & 0.74 & 0.73 & 0.74 & 0.76 & 0.79 \\
\hline $7 C-3$ & $\mathrm{U}-10 \mathrm{Mo}$ & oil & 82 & 1.53 & 1.40 & 1.30 & 1.21 & 1.11 & 1.05 & 0.97 & 0.91 & 0.88 & 0.87 & 0.83 & 0.79 & 0.78 & 0.78 & 0.76 & 0.74 & 0.74 & 0.75 & 0.78 \\
\hline 7C-4 & U-7Mo & foil & .66 & 1.46 & 1.34 & 1.25 & 1.15 & 1.11 & 1.06 & 0.99 & 0.94 & 0.91 & 0.89 & 0.87 & 0.84 & 0.84 & 0.81 & 0.80 & 0.79 & 0.76 & 0.76 & 0.78 \\
\hline 7C-5 & $\mathrm{U}-10 \mathrm{Mo}$ & foil & 1.68 & 1.45 & 1.31 & 1.23 & 1.16 & 1.09 & 1.05 & 0.99 & 0.97 & 0.91 & 0.90 & 0.86 & 0.85 & 0.83 & 0.81 & 0.80 & 0.79 & 0.77 & 0.77 & 0.77 \\
\hline $7 C-6$ & $\mathrm{U}-12 \mathrm{Mo}$ & foil & .96 & 1.60 & 1.44 & 1.32 & 1.17 & 1.09 & 1.03 & 0.96 & 0.91 & $0 . \varepsilon$ & 0.84 & 0.82 & 0.7 & 0.7 & 0.75 & 0.74 & 0.74 & 0.73 & 0.73 & 0.78 \\
\hline 7C-7 & U-7Mo & disp & 1.99 & 1.62 & 1.43 & 1.31 & 1.18 & 1.09 & 1.02 & 0.97 & 0.91 & $0 . \varepsilon$ & 0.81 & 0.79 & 0.78 & 0.76 & 0.72 & 0.71 & 0.73 & 0.73 & 0.76 & 0.83 \\
\hline $7 C-8$ & U-7Mo & disp & 1.75 & 1.47 & 1.32 & 1.24 & 1.13 & 1.07 & 1.04 & 0.98 & 0.95 & 0.5 & 0.91 & 0.87 & 0.84 & 0.82 & 0.80 & 0.78 & 0.79 & 0.76 & 0.75 & 0.79 \\
\hline $7 D-1$ & U-7Mo & disp & 1.64 & 1.40 & 1.28 & 1.19 & 1.14 & 1.07 & 1.06 & 1.03 & 1.01 & 0.95 & 0.93 & 0.88 & 0.86 & 0.83 & 0.83 & 0.81 & 0.78 & 0.77 & 0.76 & 0.78 \\
\hline 7D-2 & --- & blank & --- & --- & --- & --- & --- & --- & --- & --- & --- & --- & --- & --- & --- & --- & --- & --- & --- & --- & --- & --- \\
\hline 7D-3 & U-7Mo & disp & 1.65 & 1.41 & 1.31 & 1.23 & 1.17 & 1.11 & 1.06 & 1.01 & 1.00 & 0.94 & 0.89 & 0.90 & 0.87 & 0.84 & 0.81 & 0.78 & 0.78 & 0.76 & 0.72 & 0.75 \\
\hline 7D-4 & --- & blank & --- & --- & --- & --- & --- & --- & --- & --- & --- & --- & --- & --- & --- & --- & --- & --- & --- & --- & --- & --- \\
\hline 7D-5 & --- & blank & --- & --- & --- & --- & --- & --- & --- & --- & --- & --- & --- & --- & --- & --- & --- & --- & --- & --- & --- & --- \\
\hline 7D-6 & $\mathrm{U}-10 \mathrm{Mo}$ & foil & .75 & 1.50 & 1.36 & 1.27 & 1.17 & 1.09 & 1.06 & 1.00 & 0.98 & 0.92 & 0.88 & 0.87 & 0.83 & 0.80 & 0.79 & 0.78 & 0.75 & 0.71 & 0.72 & 0.77 \\
\hline 7D-7 & U-10Mo & foil & 1.96 & 1.57 & 1.41 & 1.27 & 1.15 & 1.06 & 1.02 & 0.96 & 0.92 & 0.88 & 0.84 & 0.81 & 0.80 & 0.80 & 0.75 & 0.74 & 0.74 & 0.74 & 0.76 & 0.82 \\
\hline 7D-8 & U-7Mo & disp & 1.80 & 1.49 & 1.34 & 1.25 & 1.13 & 1.06 & 1.02 & 0.98 & 0.96 & 0.90 & 0.89 & 0.85 & 0.85 & 0.81 & 0.78 & 0.78 & 0.76 & 0.76 & 0.77 & 0.81 \\
\hline
\end{tabular}




\section{RERTR-6}

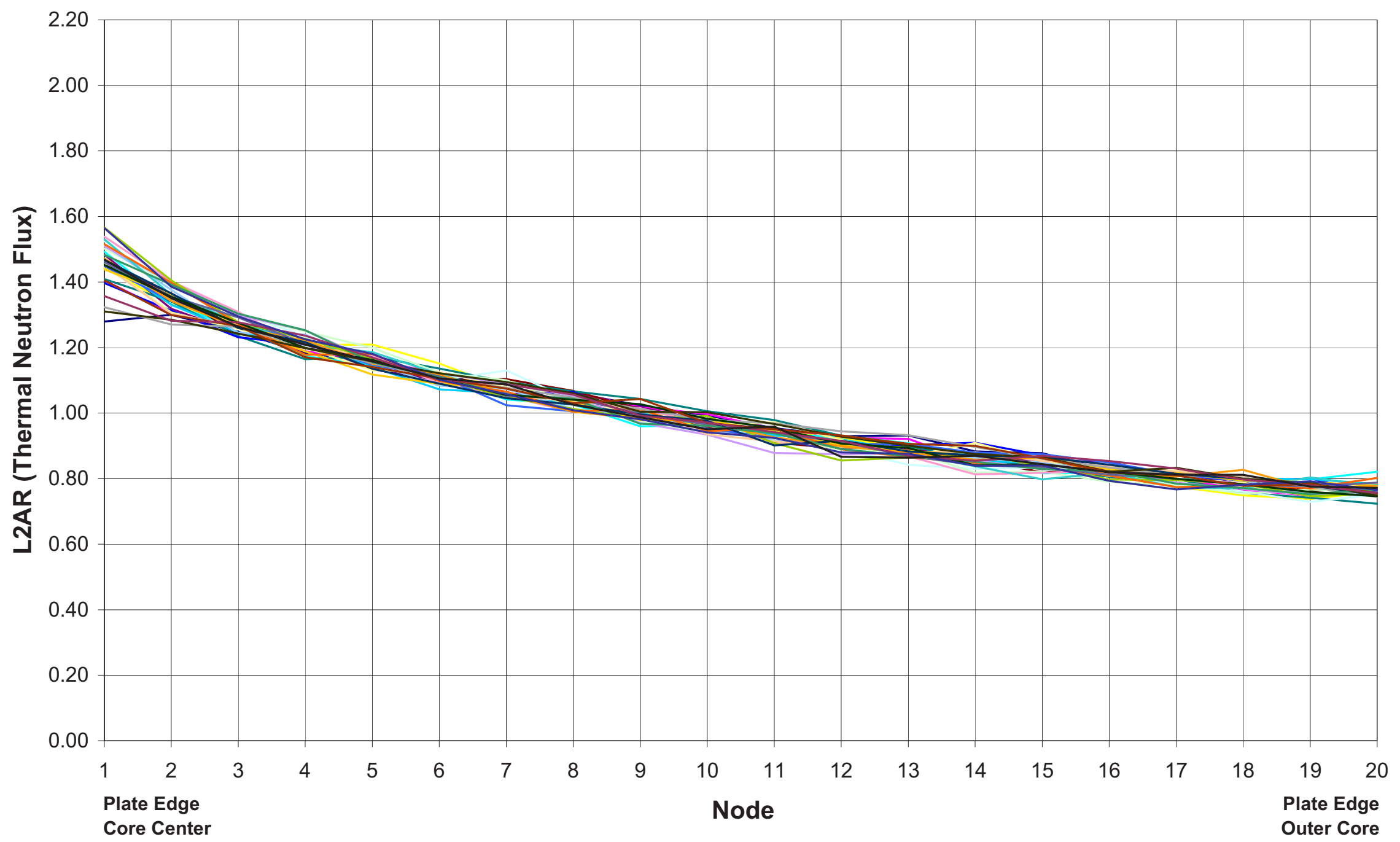




\section{RERTR-6}

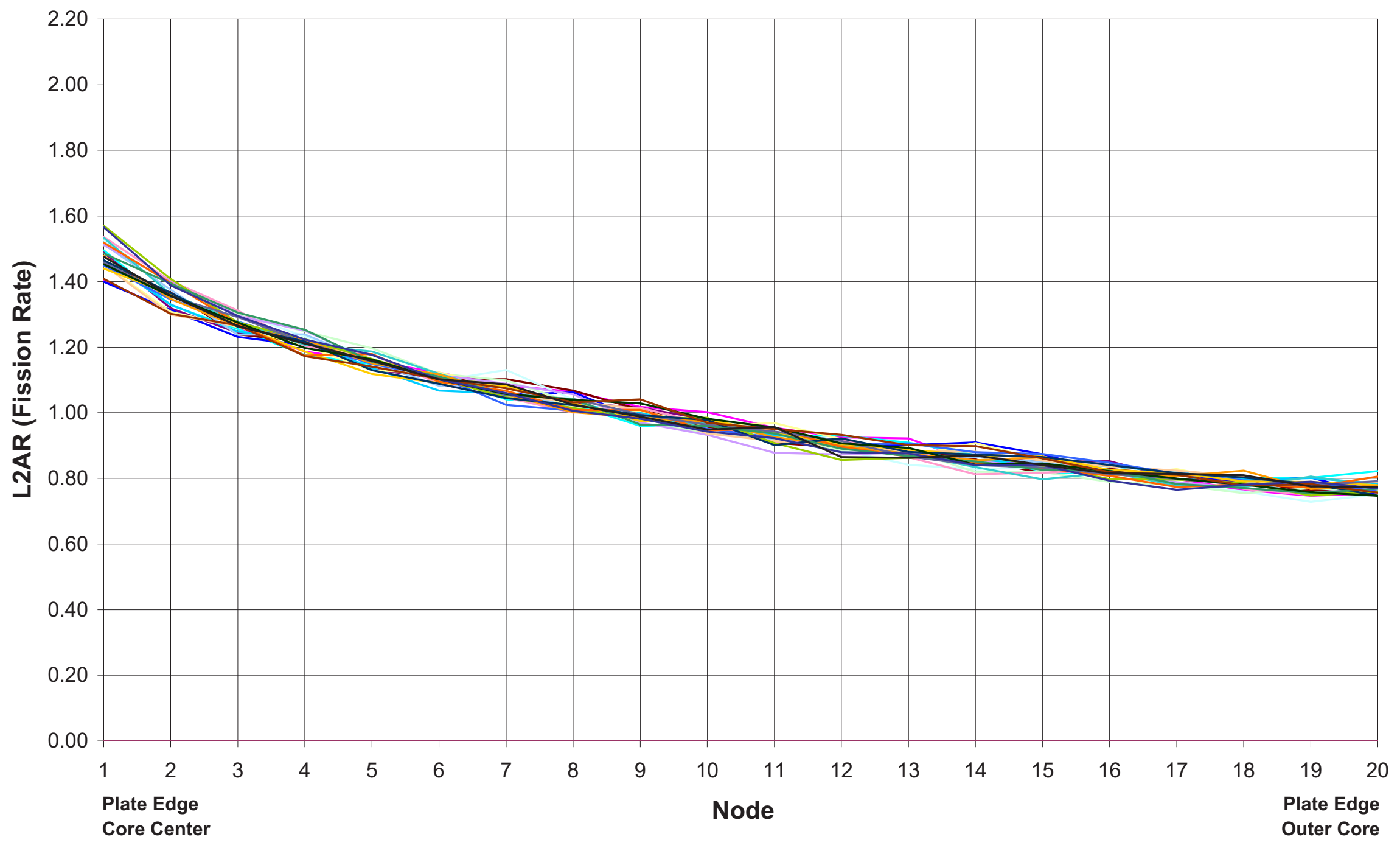




\section{RERTR-7}

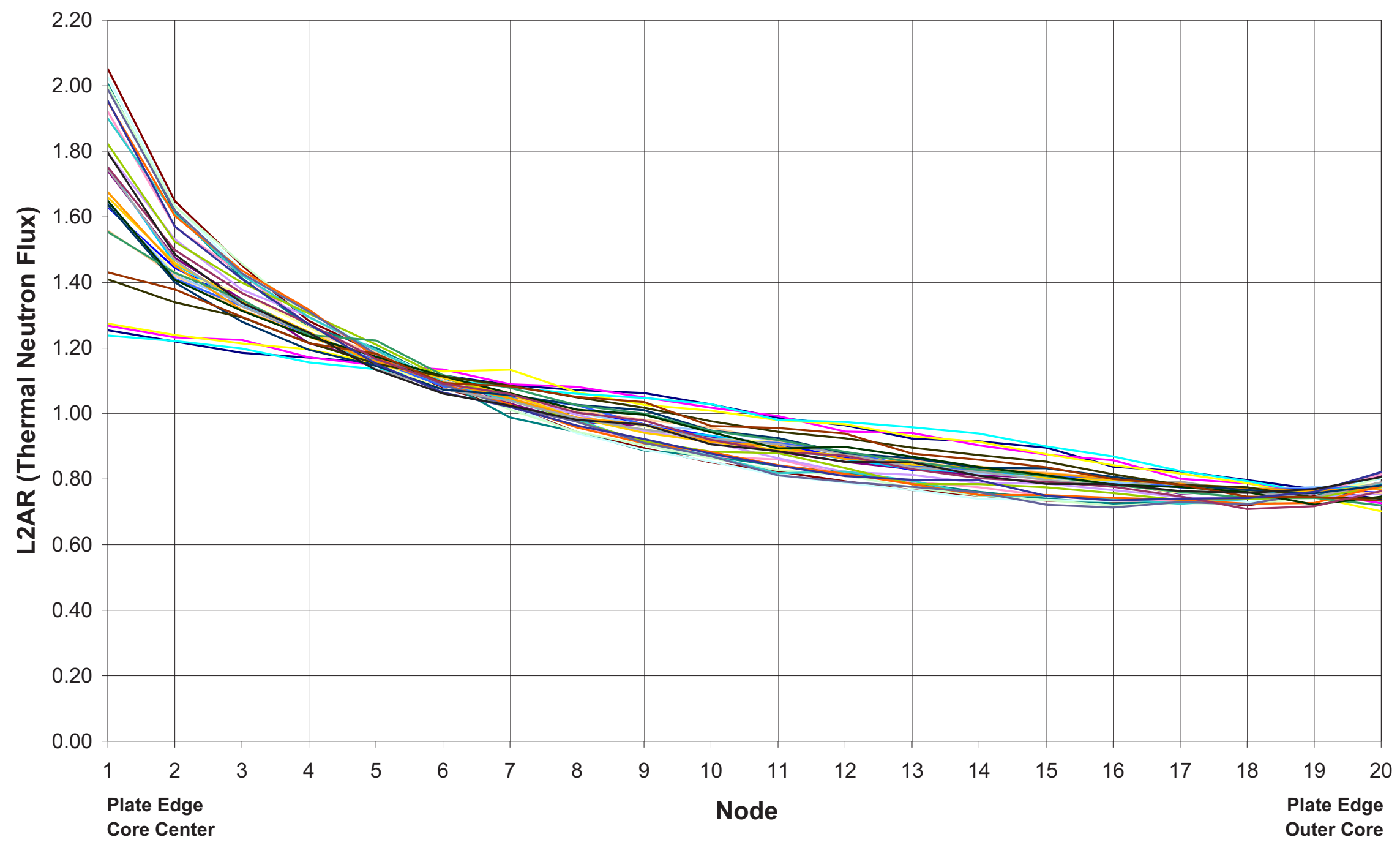




\section{RERTR-7}

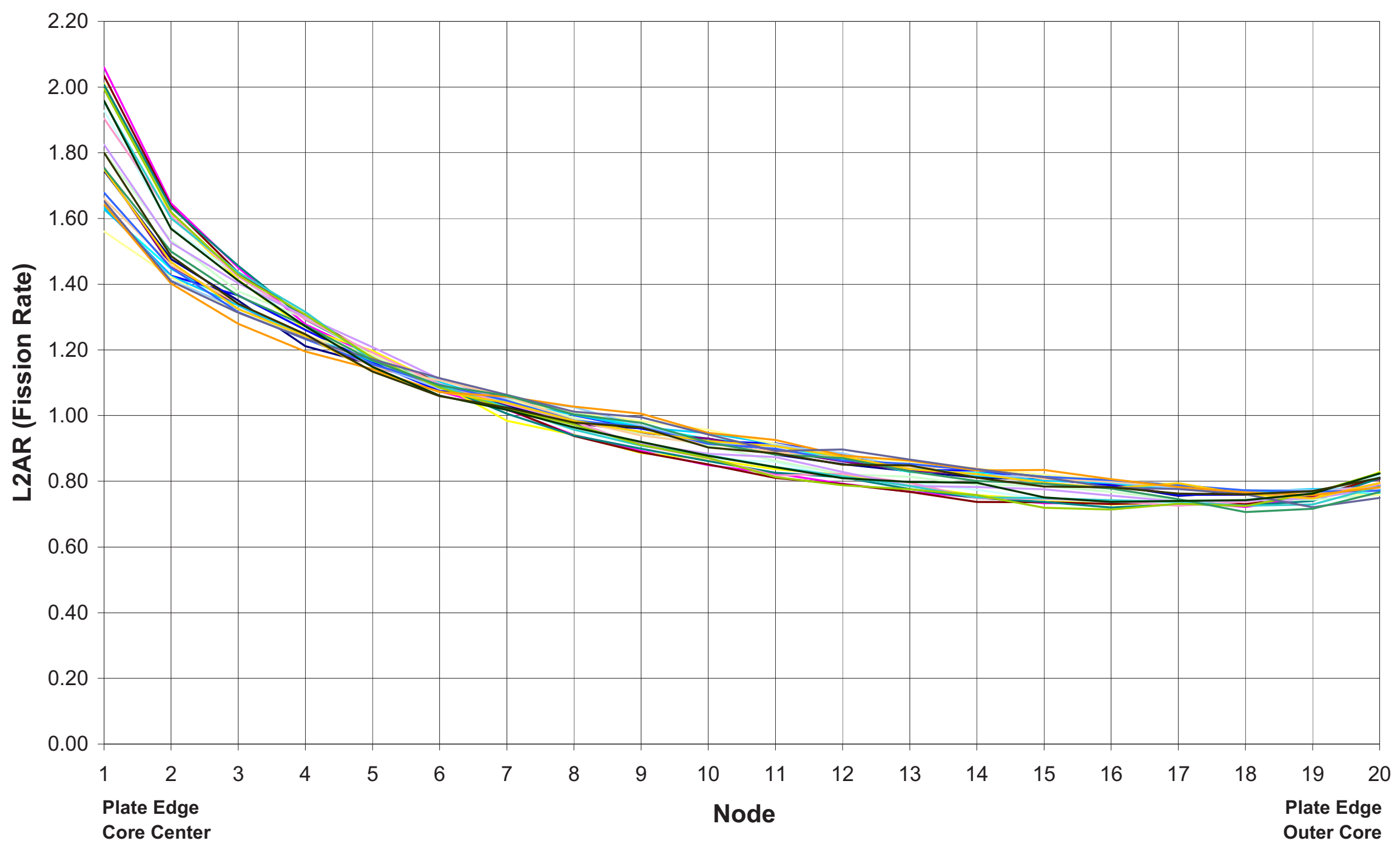




\begin{tabular}{|c|c|c|}
\hline \multirow{3}{*}{$\begin{array}{lr}\begin{array}{l}\text { Michael R } \\
\text { Finlay/FINLMRIFNINEELUS }\end{array} & \text { To } \\
12 / 19 / 200601: 07 \mathrm{PM} & \text { bcc } \\
& \text { Subject }\end{array}$} & \multicolumn{2}{|l|}{ Gray S Chang/GSCICC01/INEELUS@|NEL } \\
\hline & $\begin{array}{l}\text { Daniel M Wachs/WACHDMiCC01/NEELUS@INEL, } \\
\text { Baxter.Hayes@icp.doe.gov, } \\
\text { Adam B RobinsoniROBIABICC01/NEELUS@|NEL, }\end{array}$ & $\begin{array}{l}\hat{G} \\
\square\end{array}$ \\
\hline & power gradient across RERTR plates & \\
\hline
\end{tabular}

Hi Gray,

I have some interesting results from our gamma scans from RERTR-7. We see a large gradient in the transverse orientation decreasing from the core edge to the outer edge. We did not see such a large gradient in RERTR-6. I know there are some significant differences between RERTR- 6 \& 7 including increased enrichment and different location that may be contributing factors.

You prepared an analysis of this effect in RERTR 4 \& 5 and the ratio from core edge to outer edge was $\sim 2$. We were not able to confirm this however because the plates were rotated between cycles and therefore the gradient was largely nullified and we only performed axial gamma scans at that time.

Can I ask you to perform a similar analysis for RERTR-6 \& 7? It would be really useful to confirm the results we have. I am happy to show you what the gamma scan ratios are but feel that it would be more valuable exercise if you were to perform the analysis and not know what we have already measured.

If you have any questions on this exercise feel free to give me a call. I can also give you a job code if you need it.

Ross Finlay

RERTR Program

Materials and Fuels Complex

Idaho National Laboratory

P.O. Box 1625

Idaho Falls, ID 83415-6188

tel: (208) 533-7572

fax: (208) 533-7863 


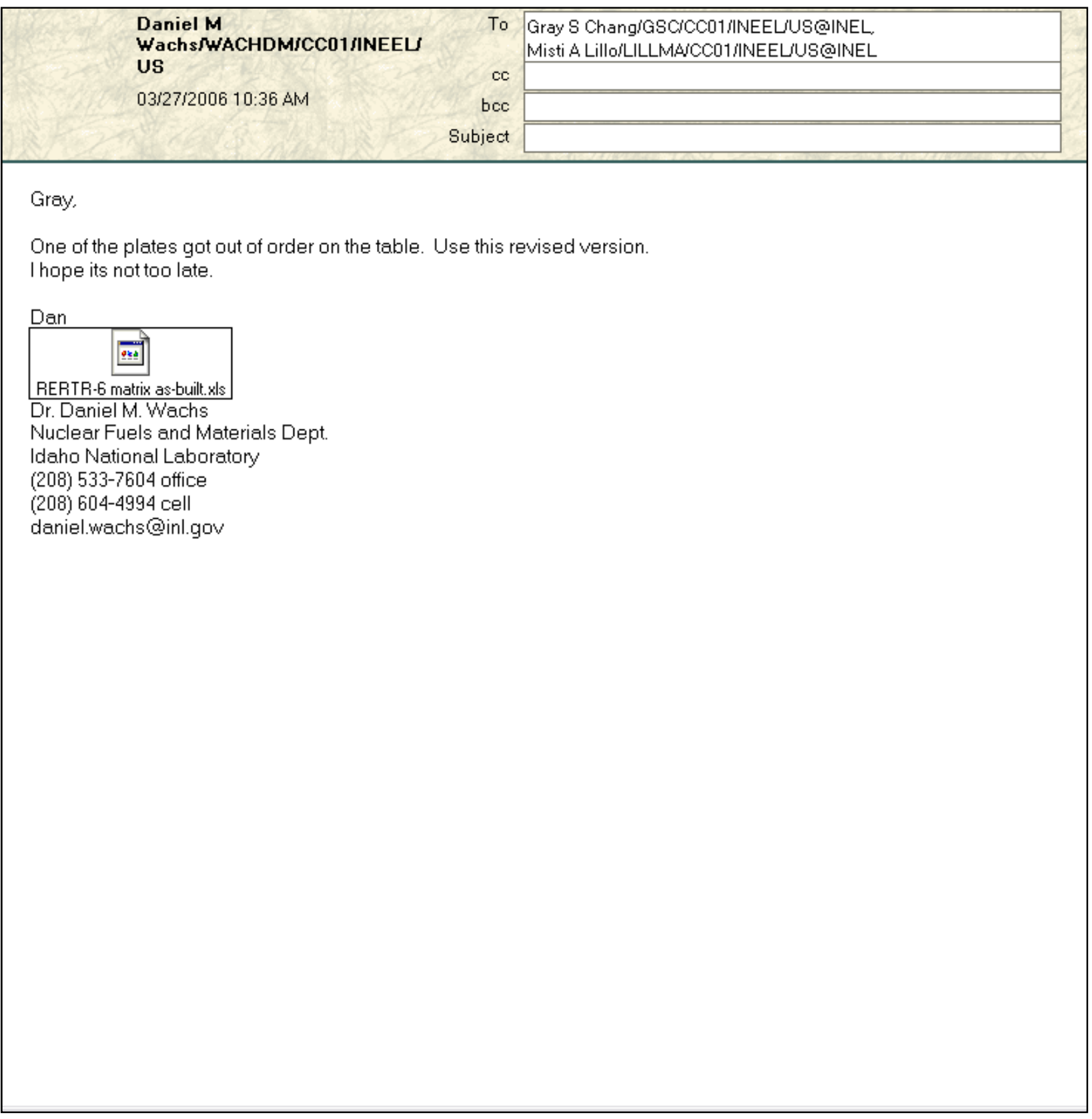


Table 1. Constituent masses for each RERTR-6 mini-plate.

\begin{tabular}{|c|c|c|c|c|c|c|c|c|c|c|c|c|c|c|c|c|c|c|c|c|c|c|c|}
\hline \multirow[b]{2}{*}{ Fuel Plate } & \multirow{2}{*}{$\begin{array}{l}\text { Fuel } \\
\text { Type }\end{array}$} & \multirow{2}{*}{$\begin{array}{l}\text { Plate } \\
\text { ID }\end{array}$} & \multirow{2}{*}{$\begin{array}{l}\text { Fuel Phase } \\
\text { Composition }\end{array}$} & \multirow{2}{*}{$\begin{array}{c}\text { Martix } \\
\text { Phase }\end{array}$} & \multirow{2}{*}{\begin{tabular}{c|} 
Foil \\
Thickness (mm)
\end{tabular}} & \multirow{2}{*}{$\begin{array}{l}\text { Fuel Alloy } \\
\text { Mass }\end{array}$} & \multicolumn{5}{|c|}{ Fuel Phase Constituent Masses (g) } & \multirow[t]{2}{*}{$\begin{array}{c}\text { Matrix Phase } \\
\text { Mass }(\mathrm{g}) \\
\end{array}$} & \multicolumn{10}{|c|}{ Matrix Phase Constituent Masses (g) } & \multirow{2}{*}{\begin{tabular}{|l|} 
Cladding \\
Mass (g) \\
$\mathrm{Al}-6061$ \\
\end{tabular}} \\
\hline & & & & & & & Total U & $\mathrm{U}-235$ & Mo & $\mathrm{Si}$ & $\mathrm{Cr}$ & & $\mathrm{Al}$ & $\mathrm{Si}$ & $\mathrm{Fe}$ & $\mathrm{Cu}$ & $\mathrm{Mn}$ & $\mathrm{Mg}$ & $\mathrm{Cr}$ & $\mathrm{Zn}$ & Ti & other & \\
\hline $6 \mathrm{~A}-1$ & blank & BLANK1 & --- & -- & --- & --- & -- & -- & -- & -- & -- & -- & -- & -- & -- & --- & -- & -- & -- & --- & -- & -- & 9.729 \\
\hline $6 \mathrm{~A}-2$ & disp & R1R010 & U-7Mo & Al-6061 & --- & 6.550 & 6.152 & 1.169 & 0.398 & -- & -- & 1.370 & 1.320 & 0.008 & 0.010 & 0.004 & 0.002 & 0.014 & 0.005 & 0.003 & 0.002 & 0.002 & 5.330 \\
\hline $6 \mathrm{~A}-3$ & blank & BLANK3 & --- & -- & --- & --- & -- & -- & -- & --- & --- & -- & --- & --- & --- & --- & --. & -.- & --- & --- & --- & --- & 9.729 \\
\hline $6 \mathrm{~A}-4$ & foil & L2F020 & U-10Mo & -- & 0.508 & 13.630 & 12.160 & 2.400 & 1.470 & -- & -- & -- & --- & --- & -- & -- & --- & -- & -- & --- & -- & -- & 6.190 \\
\hline $6 \mathrm{~A}-5$ & disp & R5R010 & U-7Mo & $\mathrm{Al}-0.5 \mathrm{Si}$ & -- & 6.530 & 6.134 & 1.165 & 0.396 & --- & -- & 1.430 & 1.423 & 0.007 & --- & --- & -- & --- & --- & --- & --- & --- & 5.330 \\
\hline $6 \mathrm{~A}-6$ & foil & L1F090 & U-10Mo & --- & 0.254 & 7.580 & 6.820 & 1.340 & 0.760 & --- & --- & --- & --- & --- & --- & --- & --- & --- & --- & --- & --. & --- & 7.960 \\
\hline $6 \mathrm{~A}-7$ & blank & BLANK4 & --- & --- & --- & --- & --- & --- & --- & --- & --- & -.- & --. & --- & --. & --- & --. & --- & --. & --- & -.- & --- & 9.729 \\
\hline $6 \mathrm{~A}-8$ & foil & NIF090 & U-7Mo & --- & 0.254 & 6.970 & 6.490 & 1.280 & 0.480 & -- & -- & --- & --- & -- & --- & --- & --- & --- & --- & --- & -- & -- & 7.960 \\
\hline 6B-1 & disp & R1R020 & U-7Mo & $\mathrm{Al}-6061$ & --- & 6.630 & 6.124 & 1.167 & 0.506 & --- & --- & 1.380 & 1.330 & 0.008 & 0.010 & 0.004 & 0.002 & 0.014 & 0.005 & 0.003 & \begin{tabular}{|l|}
0.002 \\
\end{tabular} & 0.002 & 5.330 \\
\hline 6B-2 & disp & R2R020 & U-7Mo & $\mathrm{Al}-2 \mathrm{Si}$ & --- & 6.530 & 5.944 & 1.153 & 0.586 & --- & --- & 1.420 & 1.392 & 0.028 & --- & --- & --- & --- & --- & --- & -- & --- & 5.330 \\
\hline 6B-3 & disp & V0R020 & U-10Mo & $\mathrm{Al}$ & --- & 6.810 & 6.062 & 1.164 & 0.748 & --- & --- & 1.310 & 1.31 & --- & --- & --- & --- & --. & --- & --- & --- & --- & 7.960 \\
\hline 6B-4 & foil & N1F010 & U-7Mo & --- & 0.254 & 7.150 & 6.650 & 1.310 & 0.500 & --.- & --- & --- & --- & --- & --. & --- & -- & --- & --- & --- & --. & --- & 7.960 \\
\hline 6B-5 & disp & R3R030 & U-7Mo & Al- 4043 & --- & 6.540 & 6.143 & 1.167 & 0.397 & -- & --- & 1.410 & 1.313 & 0.074 & 0.011 & 0.004 & 7E-04 & 7E-04 & -- & 0.001 & 0.003 & 0.002 & 5.330 \\
\hline 6B-6 & foil & N1F040 & U-7Mo & --- & 0.254 & 7.470 & 6.950 & 1.370 & 0.520 & --- & --- & -- & -- & --- & --- & --- & --- & --- & -- & --- & -- & --- & 7.960 \\
\hline 6B-7 & foil & LiF040 & U-10Mo & --- & 0.254 & 6.660 & 5.990 & 1.180 & 0.670 & --. & --- & -.- & -.- & --- & --- & --- & --- & --- & --- & --- & --- & --- & 7.960 \\
\hline 6B-8 & disp & V1R020 & U-10Mo & $\mathrm{Al}-6061$ & --- & 6.800 & 6.055 & 1.162 & 0.745 & --- & --- & 1.410 & 1.359 & 0.008 & 0.010 & 0.004 & 0.002 & 0.014 & 0.005 & 0.004 & 0.002 & 0.002 & 5.330 \\
\hline $6 \mathrm{C}-1$ & disp & V0R010 & U-10Mo & $\mathrm{Al}$ & --- & 6.800 & 6.058 & 1.163 & 0.742 & -- & --- & 1.320 & 1.32 & -- & --- & --- & --- & -- & --- & --- & -- & -- & 7.960 \\
\hline $6 \mathrm{C}-2$ & disp & R3R010 & U-7Mo & Al- 4043 & -- & 6.540 & 6.145 & 1.168 & 0.395 & -- & --- & 1.410 & 1.313 & 0.074 & 0.011 & 0.004 & 7E-04 & 7E-04 & -- & 0.001 & 0.003 & 0.002 & 5.330 \\
\hline $6 \mathrm{C}-3$ & disp & R2R010 & U-7Mo & $\mathrm{Al}-2 \mathrm{Si}$ & --- & 6.540 & 5.960 & 1.156 & 0.580 & --- & --- & 1.420 & 1.392 & 0.028 & --- & -- & -- & --- & --- & --- & --- & --- & 5.330 \\
\hline $6 \mathrm{C}-4$ & foil & N1F030 & U-7Mo & --- & 0.254 & 7.430 & 6.910 & 1.360 & 0.520 & --- & --- & --- & --- & --- & --. & -- & --. & -- & --- & --- & -.- & --. & 7.960 \\
\hline $6 \mathrm{C}-5$ & disp & R5R020 & U-7Mo & $\mathrm{Al}-0.5 \mathrm{Si}$ & --- & 6.530 & 6.135 & 1.166 & 0.395 & --- & --- & 1.420 & 1.413 & 0.007 & --- & --- & --- & --- & --- & --- & --. & -- & 5.330 \\
\hline 6C-6 & foil & N1F060 & U-7Mo & -- & 0.254 & 7.240 & 6.740 & 1.330 & 0.500 & -- & --- & --- & -- & -- & -- & --- & -- & -- & -- & --- & -- & -- & 7.960 \\
\hline $6 \mathrm{C}-7$ & disp & V5R030 & U-10Mo & $\mathrm{Al}-0.5 \mathrm{Si}$ & --- & 6.820 & 6.072 & 1.165 & 0.748 & --- & --- & 1.330 & 1.323 & 0.007 & --- & --- & --- & --- & -.- & --- & --- & --- & 7.960 \\
\hline $6 \mathrm{C}-8$ & blank & BLANK5 & --- & --- & --- & --- &.-- & --- & --- & --- & --- & --- & --- & --- & --. & --- & --- & --- & --. & --- & --. & -- & 9.729 \\
\hline 6D-1 & foil & L1F100 & U-10Mo & --. & 0.254 & 6.920 & 6.250 & 1.230 & 0.670 & --- & --- & -- & --- & --- & --- & --- & --- & --- & --. & --- & --. & --- & 7.960 \\
\hline 6D-2 & disp & R2R030 & U-7Mo & $\mathrm{Al}-2 \mathrm{Si}$ & --- & 6.530 & 5.951 & 1.155 & 0.579 & --. & --- & 1.420 & 1.392 & 0.028 & --- & --- & --- & --- & --- & --- & -- & --- & 5.330 \\
\hline $6 \mathrm{D}-3$ & disp & V1R010 & U-10Mo & $\mathrm{Al}-6061$ & --- & 6.790 & 6.143 & 1.160 & 0.647 & --- & --- & 1.370 & 1.320 & 0.008 & 0.010 & 0.004 & 0.002 & 0.014 & 0.005 & 0.003 & 0.002 & 0.002 & 5.330 \\
\hline 6D-4 & blank & BLANK6 & -.- & --- & --- & --- & --- & -- & --- & --- & -- & -- & --- & --- & --- & -- & --- & --- & --- & --- & --- & --- & 9.729 \\
\hline $6 \mathrm{D}-5$ & disp & R1R030 & U-7Mo & $\mathrm{Al}-6061$ & --. & 6.530 & 6.139 & 1.166 & 0.391 &.-- & --. & 1.380 & 1.330 & 0.008 & 0.010 & 0.004 & 0.002 & 0.014 & 0.005 & 0.003 & 0.002 & 0.002 & 5.330 \\
\hline 6D-6 & blank & BLANK9 & -- & -- & -- & -- & --- & -- & -- & -- & -- & -- & -- & -- & -- & -- & -- & -- & -- & --- & -- & --- & 9.729 \\
\hline 6D-7 & foil & L2F030 & U-10Mo & -- & 0.508 & 13.280 & 11.850 & 2.340 & 1.430 & -- & -- & -- & -- & -- & --- & -- & --- & -- & -- & --- & -- & -- & 6.190 \\
\hline 6D-8 & disp & V5R020 & U-10Mo & $\mathrm{Al}-0.5 \mathrm{Si}$ & -- & 6.790 & 6.050 & 1.161 & 0.740 & -- & -- & 1.320 & 1.313 & 0.007 & -- & -- & -- & -- & -- & $\ldots$ & -- & - & 7.960 \\
\hline & & & & & & & 174.077 & 33.747 & 16.513 & 0.000 & 0.000 & 22.120 & 21.561 & \begin{tabular}{|l|l|}
0.302 \\
\end{tabular} & \begin{tabular}{|l|}
0.071 \\
\end{tabular} & \begin{tabular}{|l|l|}
0.027 \\
\end{tabular} & \begin{tabular}{|l|l|}
0.012 \\
\end{tabular} & \begin{tabular}{|l|l|}
0.071 \\
\end{tabular} & \begin{tabular}{|l|}
0.026 \\
\end{tabular} & 0.020 & 0.016 & 0.015 & 230.234 \\
\hline
\end{tabular}


Table 2. Constituent densities for RERTR-6 mini-plates based on nominal fuel meat volume.

\begin{tabular}{|c|c|c|c|c|c|c|c|c|c|c|c|c|c|c|c|c|c|c|}
\hline \multirow[b]{2}{*}{ Fuel Plate } & \multirow{2}{*}{$\begin{array}{l}\text { Fuel Phase } \\
\text { Composition }\end{array}$} & \multirow{2}{*}{$\begin{array}{l}\text { Fuel Meat } \\
\text { Volume (cc) }\end{array}$} & \multicolumn{5}{|c|}{ Fuel Phase Constituent Densities $(\mathrm{g} / \mathrm{cm} 3)$} & \multirow{2}{*}{\begin{tabular}{|c|} 
Matrix Phase \\
Dens. $(\mathrm{g} / \mathrm{cm} 3)$ \\
$\mathrm{Al}$
\end{tabular}} & \multicolumn{10}{|c|}{ Matrix Phase Constituent Densities $(\mathrm{g} / \mathrm{cc})$} \\
\hline & & & Total U & $\mathrm{U}-235$ & Mo & $\mathrm{Si}$ & $\mathrm{Cr}$ & & $\mathrm{Al}$ & $\mathrm{Si}$ & $\mathrm{Fe}$ & $\mathrm{Cu}$ & $\mathrm{Mn}$ & $\mathrm{Mg}$ & $\mathrm{Cr}$ & $\mathrm{Zn}$ & $\mathrm{Ti}$ & other \\
\hline $6 \mathrm{~A}-1$ & --- & --- & --- & --- & --- & --- & --- & --- & --- & --- & --- & --- & --- & --- & --- & --- & --- & --- \\
\hline $6 \mathrm{~B}-5$ & U-7Mo & 0.957 & 6.428 & 1.222 & 0.416 & -- & --- & 1.432 & 1.379 & 0.009 & 0.010 & 0.004 & 0.002 & 0.014 & 0.005 & 0.004 & 0.002 & 0.002 \\
\hline $6 \mathrm{~A}-3$ & --- & --- & --- & --- & --- & --- & --- & --- & --- & --- & --- & --- & --- & --- & --- & --- & --- & --- \\
\hline $6 \mathrm{~A}-4$ & U-10Mo & 0.794 & 15.306 & 3.021 & 1.850 & --- & --- & --- & --- & --- & --- & --- & --- & --- & --- & --- & --- & --- \\
\hline $6 \mathrm{~A}-5$ & U-7Mo & 0.957 & 6.410 & 1.217 & 0.414 & --- & --- & 1.494 & 1.487 & 0.007 & --- & --- & --- & --- & --- & --- & --- & --- \\
\hline $6 \mathrm{~A}-6$ & U-10Mo & 0.397 & 17.169 & 3.373 & 1.913 & --- & --- & --- & --- & --- & --- & --- & --- & --- & --- & --- & --- & --- \\
\hline $6 \mathrm{~A}-7$ & --- & --- & --- & --- & --- & --- & --- & --- & --- & --- & --- & --- & --- & --- & --- & --- & --- & --- \\
\hline $6 \mathrm{~A}-8$ & U-7Mo & 0.397 & 16.338 & 3.222 & 1.208 & --- & --- & --- & --- & --- & --- & --- & --- & --- & --- & --- & --- & --- \\
\hline 6B-1 & U-7Mo & 0.957 & 6.399 & 1.219 & 0.529 & --- & --- & 1.442 & 1.389 & 0.009 & 0.010 & 0.004 & 0.002 & 0.014 & 0.005 & 0.004 & 0.002 & 0.002 \\
\hline $6 \mathrm{~B}-2$ & U-7Mo & 0.957 & 6.211 & 1.205 & 0.612 & --- & --- & 1.484 & 1.454 & 0.030 & --- & --- & --- & --- & --- & --- & --- & --- \\
\hline $6 \mathrm{~B}-3$ & U-10Mo & 0.957 & 6.334 & 1.216 & 0.782 & --- & --- & --- & --- & --- & --- & --- & --- & --- & --- & --- & --- & --- \\
\hline 6B-4 & U-7Mo & 0.397 & 16.741 & 3.298 & 1.259 & --- & --- & --- & --- & --- & --- & --- & --- & --- & --- & --- & --- & --- \\
\hline 6B-5 & U-7Mo & 0.957 & 6.419 & 1.219 & 0.415 & --- & --- & 1.473 & 1.372 & 0.077 & 0.012 & 0.004 & 0.001 & --- & --- & 0.001 & --- & 0.002 \\
\hline 6B-6 & U-7Mo & 0.397 & 17.496 & 3.449 & 1.309 & -- & --- & --- & --- & --- & --- & --- & --- & --- & --- & --- & --- & --- \\
\hline 6B-7 & U-10Mo & 0.397 & 15.079 & 2.971 & 1.687 & --- & --- & --- & --- & --- & --- & --- & --- & --- & --- & --- & --- & --- \\
\hline 6B-8 & U-10Mo & 0.957 & 6.327 & 1.214 & 0.778 & --- & --- & 1.473 & 1.420 & 0.009 & 0.010 & 0.004 & 0.002 & 0.015 & 0.006 & 0.004 & 0.002 & 0.002 \\
\hline $6 \mathrm{C}-1$ & U-10Mo & 0.957 & 6.330 & 1.215 & 0.775 & -- & --- & --- & --- & --- & --- & --- & --- & --- & --- & --- & --- & --- \\
\hline $6 \mathrm{C}-2$ & U-7Mo & 0.957 & 6.421 & 1.220 & 0.413 & --- & --- & 1.473 & 1.372 & 0.077 & 0.012 & 0.004 & 0.001 & --- & --- & 0.001 & --- & 0.002 \\
\hline $6 \mathrm{C}-3$ & U-7Mo & 0.957 & 6.228 & 1.208 & 0.606 & -- & --- & 1.484 & 1.454 & 0.030 & --- & --- & --- & --- & --- & --- & --- & --- \\
\hline 6C-4 & U-7Mo & 0.397 & 17.395 & 3.424 & 1.309 & -- & --- & --- & --- & --- & --- & --- & --- & --- & --- & --- & --- & -- \\
\hline $6 \mathrm{C}-5$ & U-7Mo & 0.957 & 6.411 & 1.218 & 0.413 & --- & --- & 1.484 & 1.476 & 0.007 & --- & --- & --- & --- & --- & --- & --- & --- \\
\hline $6 \mathrm{C}-6$ & U-7Mo & 0.397 & 16.967 & 3.348 & 1.259 & --- & --- & --- & --- & --- & --- & --- & --- & --- & --- & --- & --- & --- \\
\hline $6 \mathrm{~A}-7$ & U-10Mo & 0.957 & 6.345 & 1.217 & 0.782 & --- & --- & 1.390 & 1.383 & 0.007 & --- & --- & --- & --- & --- & --- & --- & -- \\
\hline 6C-8 & --- & --- & --- & --- & --- & --- & --- & --- & --- & --- & --- & --- & --- & --- & --- & --- & --- & --- \\
\hline $6 \mathrm{D}-1$ & U-10Mo & 0.397 & 15.734 & 3.096 & 1.687 & --- & --- & --- & --- & --- & --- & --- & --- & --- & --- & --- & --- & --- \\
\hline $6 \mathrm{C}-3$ & U-7Mo & 0.957 & 6.218 & 1.207 & 0.605 & -- & --- & 1.484 & 1.454 & 0.030 & --- & --- & --- & --- & --- & --- & --- & --- \\
\hline $6 \mathrm{D}-3$ & U-10Mo & 0.957 & 6.419 & 1.212 & 0.676 & --- & --- & 1.432 & 1.379 & 0.009 & 0.010 & 0.004 & 0.002 & 0.014 & 0.005 & 0.004 & 0.002 & 0.002 \\
\hline $6 \mathrm{D}-4$ & --- & --- & --- & --- & --- & --- & --- & --- & --- & --- & --- & --- & --- & --- & --- & --- & --- & --- \\
\hline 6B-5 & U-7Mo & 0.957 & 6.415 & 1.218 & 0.409 & -- & --- & 1.442 & 1.389 & 0.009 & 0.010 & 0.004 & 0.002 & 0.014 & 0.005 & 0.004 & 0.002 & 0.002 \\
\hline $6 \mathrm{D}-6$ & --- & --- & --- & --- & --- & --- & --- & --- & --- & --- & --- & --- & --- & --- & --- & -- & --- & --- \\
\hline $6 \mathrm{D}-7$ & U-10Mo & 0.794 & 14.916 & 2.945 & 1.800 & --- & --- & --- & --- & --- & --- & --- & --- & --- & --- & --- & --- & --- \\
\hline $6 \mathrm{D}-8$ & U-10Mo & 0.957 & 6.322 & 1.213 & 0.773 & --- & --- & 1.379 & 1.37241 & 0.0069 & --- & --- & --- & --- & --- & --- & --- & --- \\
\hline
\end{tabular}




\section{Idaho}

National

Laboratory

Intra-Laboratory Memorandum

March 6, 2006

DMW-002-06

TO: $\quad$ Distribution

FROM: D. M.Wachs Nuclear Fuels and Materials Dept.

\section{SUBJECT: As-Built Constituent Densities for the RERTR-7A Mini-plate Experiment}

The constituent density of each element included in the RERTR-7A experiment is calculated to enable neutronic analysis. As-built mass data was collected for each plate and has been smeared over the nominal fuel meat volume. The experiment matrix is shown in Table 1.

\begin{tabular}{|c|c|c|c|c|}
\hline \multicolumn{5}{|c|}{ RERTR-7A Experiment MatrixŃ As Loaded } \\
\hline Capsule & Column 1 & Column 2 & Column 3 & Column 4 \\
\hline A-Top & $\begin{array}{c}\text { A1 } \\
\text { DUM11 } \\
\text { BLANK }\end{array}$ & $\begin{array}{c}\text { A2 } \\
\text { DUM14 } \\
\text { BLANK }\end{array}$ & $\begin{array}{c}\text { A3 } \\
\text { DUM12 } \\
\text { BLANK }\end{array}$ & $\begin{array}{c}\text { A4 } \\
\text { DUM8 } \\
\text { BLANK }\end{array}$ \\
\hline \multirow[t]{2}{*}{ A-Bottom } & A5 & A6 & A7 & A8 \\
\hline & $\begin{array}{c}\text { R3R040 } \\
\text { U-7Mo Roll } \\
\text { Al } 4043 \text { Matrix }\end{array}$ & $\begin{array}{c}\text { V5R040 } \\
\text { U-7Mo Roll } \\
\text { Al-0.5Si Matrix }\end{array}$ & $\begin{array}{c}\text { R5R030 } \\
\text { U-10Mo Roll } \\
\text { Al-0.5 Si Matrix }\end{array}$ & $\begin{array}{c}\text { H1F020 } \\
\text { U-12Mo FSW } \\
\text { 0.010" Foil }\end{array}$ \\
\hline \multirow{2}{*}{ B-Top } & B1 & $\mathrm{B} 2$ & B3 & B4 \\
\hline & $\begin{array}{c}\text { R1R040 } \\
\text { U-7Mo Roll } \\
\text { Al } 6061 \text { Matrix }\end{array}$ & $\begin{array}{c}\text { R2R040 } \\
\text { U-7Mo Roll } \\
\text { Al-2Si Matrix }\end{array}$ & $\begin{array}{c}\text { R0R010 } \\
\text { U-7Mo Roll } \\
\text { Pure Al Matrix }\end{array}$ & $\begin{array}{c}\text { H1T010 } \\
\text { U-12Mo TLPB } \\
\text { 0.010" Foil }\end{array}$ \\
\hline \multirow[t]{2}{*}{ B-Bottom } & B5 & B6 & B7 & B8 \\
\hline & $\begin{array}{c}\text { L1F01L } \\
\text { U-10Mo FSW } \\
\text { Holed Foil }\end{array}$ & $\begin{array}{c}\text { V5R050 } \\
\text { U-7Mo Roll } \\
\text { Al-0.5Si Matrix }\end{array}$ & $\begin{array}{c}\text { L1F140 } \\
\text { U-10Mo FSW } \\
\text { 0.010" Foil }\end{array}$ & $\begin{array}{c}\text { MZ25 } \\
\text { U-7Mo Roll } \\
\text { Zr Clad CNEA }\end{array}$ \\
\hline \multirow[t]{2}{*}{ C-Top } & $\mathrm{C} 1$ & $\mathrm{C} 2$ & $\mathrm{C} 3$ & $\mathrm{C} 4$ \\
\hline & $\begin{array}{c}\text { H1F030 } \\
\text { U-12Mo FSW } \\
\text { 0.010" Foil }\end{array}$ & $\begin{array}{c}\text { L1T020 } \\
\text { U-10Mo TLPB } \\
\text { 0.010" Foil }\end{array}$ & $\begin{array}{c}\text { L1F110 } \\
\text { U-10Mo FSW } \\
\text { 0.010" Foil }\end{array}$ & $\begin{array}{c}\text { MZ50 } \\
\text { U-7Mo Roll } \\
\text { Zr Clad CNEA }\end{array}$ \\
\hline \multirow[t]{2}{*}{ C-Bottom } & $\mathrm{C} 5$ & $\mathrm{C} 6$ & $\mathrm{C} 7$ & $\mathrm{C} 8$ \\
\hline & $\begin{array}{c}\text { L1F120 } \\
\text { U-10Mo FSW } \\
\text { 0.010" Foil }\end{array}$ & $\begin{array}{c}\text { H1T020 } \\
\text { U-12Mo TLPB } \\
\text { 0.010" Foil }\end{array}$ & $\begin{array}{c}\text { R3R050 } \\
\text { U-7Mo Roll } \\
\text { Al } 4043 \text { Matrix }\end{array}$ & $\begin{array}{c}\text { R5R040 } \\
\text { U-10Mo Roll } \\
\text { Al-0.5 Si Matrix }\end{array}$ \\
\hline D-Top & $\begin{array}{c}\text { D1 } \\
\text { R1R050 } \\
\text { U-7Mo Roll } \\
\text { Al 6061 Matrix } \\
\end{array}$ & $\begin{array}{c}\text { D2 } \\
\text { DUM13 } \\
\text { BLANK }\end{array}$ & $\begin{array}{c}\text { D3 } \\
\text { R0R020 } \\
\text { U-7Mo Roll } \\
\text { Pure Al Matrix }\end{array}$ & $\begin{array}{c}\text { D4 } \\
\text { DUM19 } \\
\text { BLANK }\end{array}$ \\
\hline \multirow[t]{2}{*}{ D-Bottom } & D5 & D6 & D7 & D8 \\
\hline & $\begin{array}{l}\text { DUM05 } \\
\text { BLANK }\end{array}$ & $\begin{array}{c}\text { L1F160 } \\
\text { U-10Mo FSW } \\
0.010 " \text { Foil }\end{array}$ & $\begin{array}{c}\text { L2F040 } \\
\text { U-10Mo TLPB } \\
0.020 " \text { Foil }\end{array}$ & $\begin{array}{c}\text { R2R050 } \\
\text { U-7Mo Roll } \\
\text { Al-2Si Matrix }\end{array}$ \\
\hline
\end{tabular}

Table 1. RERTR-7A experiment matrix.

The mass of fuel alloy included in each plate is based on measurements performed during fabrication. Prior to assembling the monolithic plates, the weight of the foil is recorded. 
This value is therefore used as a reliable reference for fuel alloy mass. The masses of both the fuel alloy and matrix material are recorded prior to pellet pressing. The final pellet mass is typically less than the total of the two masses and mass losses are assumed to be equally divided (by volume) between the matrix and fuel alloy. These final compact masses and the assumed component masses are recorded in the as-built data package and are used as the reference in this document. The foil and compact masses are tabulated in Table 2.

\begin{tabular}{|c|c|c|c|c|c|c|c|c|}
\hline $\begin{array}{l}\text { Fuel } \\
\text { Plate }\end{array}$ & $\begin{array}{l}\text { Fuel } \\
\text { Type }\end{array}$ & Plate ID & $\begin{array}{c}\text { Fuel Phase } \\
\text { Comp. }\end{array}$ & $\begin{array}{l}\text { Matrix } \\
\text { Phase }\end{array}$ & $\begin{array}{c}\text { Foil } \\
\text { Mass } \\
(\mathrm{gm})\end{array}$ & $\begin{array}{c}\text { Fuel } \\
\text { Comp. } \\
\text { Mass } \\
(\mathrm{gm})\end{array}$ & $\begin{array}{l}\text { Fuel } \\
\text { Alloy } \\
\text { Mass } \\
\text { (gm) }\end{array}$ & $\begin{array}{c}\text { Matrix } \\
\text { Mass } \\
(\mathrm{gm})\end{array}$ \\
\hline A-1 & blank & --- & --- & --- & --- & --- & -- & --- \\
\hline A-2 & blank & --- & --- & --- & --- & --- & --- & --- \\
\hline A-3 & blank & --- & --- & --- & --- & --- & --- & --- \\
\hline A-4 & blank & --- & --- & --- & --- & --- & --- & --- \\
\hline A-5 & disp & R3R040 & U-7Mo & Al-4043 & --- & 7.95 & 6.521 & 1.431 \\
\hline A-6 & disp & V5R040 & U-10Mo & $\mathrm{Al}-0.5 \mathrm{Si}$ & --- & 8.18 & 6.807 & 1.369 \\
\hline A-7 & disp & R5R030 & U-7Mo & Al-0.5 Si & --- & 7.96 & 6.535 & 1.422 \\
\hline A-8 & foil & H1F020 & U-12Mo & --- & 6.43 & --- & --- & --- \\
\hline B-1 & disp & R1R040 & U-7Mo & Al-6061 & --- & 7.96 & 6.580 & 1.380 \\
\hline B-2 & disp & R2R040 & U-7Mo & Al-2 Si & --- & 7.95 & 6.531 & 1.420 \\
\hline B-3 & disp & R0R010 & U-7Mo & $\mathrm{Al}$ & --- & 7.95 & 6.533 & 1.422 \\
\hline B-4 & foil & H1T010 & U-12Mo & --- & 6.27 & --- & --- & --- \\
\hline B-5 & foil & L1FOIL* & U-10Mo & --- & 5.90 & --- & --- & --- \\
\hline B-6 & disp & V5R050 & U-10Mo & Al-0.5 Si & --- & 8.15 & 6.782 & 1.372 \\
\hline B-7 & foil & L1F140 & U-10Mo & --- & 6.52 & --- & --- & --- \\
\hline B-8 & foil & MZ25 & U-7Mo & --- & 6.35 & --- & --- & --- \\
\hline C-1 & foil & H1F030 & U-12Mo & -- & 6.62 & --- & --- & --- \\
\hline C-2 & foil & L1T020 & U-10Mo & -- & 6.39 & --- & --- & --- \\
\hline C-3 & foil & L1F110 & U-10Mo & -- & 6.57 & --- & --- & --- \\
\hline C-4 & foil & MZ50 & U-7Mo & --- & 11.90 & --- & --- & --- \\
\hline C-5 & foil & L1F120 & U-10Mo & -- & 6.62 & --- & --- & --- \\
\hline C-6 & foil & H1T020 & U-12Mo & --- & 6.15 & --- & --- & --- \\
\hline C-7 & disp & R3R050 & U-7Mo & Al-4043 & --- & 7.97 & 6.535 & 1.432 \\
\hline C-8 & disp & R5R040 & U-7Mo & Al-0.5 Si & --- & 7.96 & 6.526 & 1.426 \\
\hline D-1 & disp & R1R050 & U-7Mo & Al-6061 & --- & 7.92 & 6.538 & 1.380 \\
\hline D-2 & blank & --- & --- & --- & --- & --- & --- & --- \\
\hline D-3 & disp & R0R020 & U-7Mo & $\mathrm{Al}$ & --- & 7.95 & 6.528 & 1.420 \\
\hline D-4 & blank & --- & --- & --- & --- & --- & --- & --- \\
\hline D-5 & blank & --- & --- & --- & --- & --- & --- & --- \\
\hline D-6 & foil & L1F160 & U-10Mo & --- & 5.41 & --- & --- & --- \\
\hline D-7 & foil & L2F040 & U-10Mo & --- & 12.04 & --- & --- & --- \\
\hline D-8 & disp & R2R050 & U-7Mo & Al-2 Si & --- & 7.95 & 6.529 & 1.418 \\
\hline
\end{tabular}

*Holed Foil

Table 2. Fuel meat masses from as-built data.

The amount of fuel alloy and matrix material incorporated into each fuel compact is set to achieve the target uranium density of $6 \mathrm{~g}-\mathrm{U} / \mathrm{cc}$. Consequently, fuel alloys with higher molybdenum content require a higher fuel alloy volume fraction. The data used to calculate the 
target volume fraction for both fuel phases is shown in Table 3. These values are used during scoping analysis of each experiment until as-built data is available.

\begin{tabular}{|c|c|c|c|c|c|c|c|c|}
\hline & $\begin{array}{c}\text { Alloy } \\
\text { Density } \\
(\mathbf{g} / \mathbf{c m} 3)\end{array}$ & $\begin{array}{c}\text { Alloy U } \\
\text { Density } \\
(\mathbf{g} / \mathbf{c m} 3)\end{array}$ & $\begin{array}{c}\text { Volume } \\
\text { \% Alloy }\end{array}$ & $\begin{array}{c}\text { Alloy } \\
\text { Mass } \\
(\mathbf{g})\end{array}$ & $\begin{array}{c}\text { Matrix } \\
\text { Mass } \\
(\mathbf{g})\end{array}$ & $\begin{array}{c}\text { Fuel } \\
\text { Meat } \\
\text { Mass } \\
(\mathbf{g})\end{array}$ & $\begin{array}{c}\text { U Mass } \\
(\mathbf{g})\end{array}$ & $\begin{array}{c}\text { Mo } \\
\text { Mass } \\
(\mathbf{g})\end{array}$ \\
\hline U-7Mo & 17.53 & 16.31 & $36.8 \%$ & 6.172 & 1.633 & 7.805 & 5.742 & 0.432 \\
\hline U-10Mo & 17.02 & 15.32 & $39.2 \%$ & 6.379 & 1.572 & 7.951 & 5.742 & 0.574 \\
\hline
\end{tabular}

Table 3. Dispersion fuel masses for fuel meat loading targets of $6.0 \mathrm{~g}-\mathrm{U} / \mathrm{cc}$.

The fuel alloy used in each mini-plate is based on a nominal target composition (either U-7Mo or U-10Mo in wt\%). The alloy is fabricated by blending known amounts of uranium and molybdenum. Samples from each alloy batch are examined in the MFC Analytical $\mathrm{Lab}$ to determine the uranium isotopics and composition. The isotopic analysis yielded a uranium enrichment of 58\%. The molybdenum content typically varies less than $0.5 \%$ from the nominal target and nominal values are used to calculate the uranium and molybdenum content in each plate from the fuel alloy content.

A variety of different matrix materials are used in the mini-plates including aluminum, Al-6061, Al-4043, Al-0.5 Si, and Al-2.0 Si. The composition of the aluminum alloys vary but average values are used to estimate the amount of minor elements incorporated into the fuel meat of each plate. The composition (in $\mathrm{wt} \%$ ) of matrix component is summarized in the Table 4.

\begin{tabular}{|c|c|c|c|c|c|c|c|c|c|c|}
\hline $\begin{array}{c}\text { Matrix } \\
\text { Compositions }\end{array}$ & Al & Si & Fe & Cu & Mn & Mg & Cr & Zn & Ti & other \\
\hline Al-6061 & 96.35 & 0.6 & 0.7 & 0.275 & 0.1 & 1.0 & 0.375 & 0.25 & 0.15 & 0.15 \\
\hline Al-4043 & 93.1 & 5.25 & 0.8 & 0.3 & 0.05 & 0.05 & & 0.1 & 0.2 & 0.15 \\
\hline Al-2.0Si & 80 & 20 & & & & & & & & \\
\hline Al-0.5Si & 95 & 5 & & & & & & & & \\
\hline Al & 100 & & & & & & & & & \\
\hline
\end{tabular}

Table 4. Compositions of matrix materials used in dispersion fuel meats (in wt\%).

Based on this information the constituent masses for each component are calculated for each mini-plate and are shown in Table 5. The constituent densities are then calculated and shown in Table 6 based on the nominal fuel meat volumes of $0.397 \mathrm{~cm} 3,0.794 \mathrm{~cm} 3$, and 0.957 $\mathrm{cm} 3$ for monolithic, thick monolithic, and dispersion, respectively. 


\begin{tabular}{|c|c|c|c|c|c|c|c|c|c|c|c|c|c|c|c|c|}
\hline \multirow[b]{2}{*}{ Fuel Plate } & \multirow{2}{*}{$\begin{array}{l}\text { Fuel } \\
\text { Type }\end{array}$} & \multirow{2}{*}{$\begin{array}{l}\text { Plate } \\
\text { ID }\end{array}$} & \multirow{2}{*}{\multicolumn{2}{|c|}{$\begin{array}{l}\text { Fuel Phase } \\
\text { Composition }\end{array}$}} & \multirow{2}{*}{\multicolumn{2}{|c|}{$\begin{array}{r}\text { Martix } \\
\text { Phase }\end{array}$}} & & & & uel Meat & & & uel Phase & e Constituen & Masses ( & \\
\hline & & & & & & & Thickn & ness $(\mathrm{mm})$ & & Mass & & rotal U & $\mathrm{U}-235$ & Mo & $\mathrm{Si}$ & $\mathrm{Cr}$ \\
\hline $\mathrm{A}-1$ & blank & --- & - & & 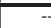 & $=$ & & --- & & -- & & --- & -- & --- & --- & --- \\
\hline A-2 & blank & -.- & $\ldots$ & & -. & - & & --- & & -.- & & --. & -.- & -.- & --- & --- \\
\hline A-3 & blank & --. & $\ldots$ & & .. & -. & & -.- & & -.- & & .-. & -.. & .-. & -.. & -.- \\
\hline A-4 & blank & --- & $\ldots$ & & $\ldots$ & - & & --- & & --- & & -- & $\cdots$ & --- & -- & -- \\
\hline A-5 & disp & R3R040 & U-71 & & $\mathrm{Al}-4$ & 4043 & & -.- & & 7.950 & & 6.060 & 3.530 & 0.459 & -.- & --- \\
\hline A-6 & disp & V5R040 & U-10 & & $\mathrm{Al}-0$ & $1.5 \mathrm{Si}$ & & --- & & 8.180 & & 6.130 & 3.550 & 0.681 & .-. & ... \\
\hline A-7 & disp & R5R030 & U-71 & & $\mathrm{Al}-0$ & $.5 \mathrm{Si}$ & & .-- & & 7.96 & & 6.080 & 3.54 & 0.458 & -.- & --. \\
\hline A-8 & foil & H1F020 & U-12 & & . & - & & .254 & & 6.430 & & 5.650 & 3.330 & 0.780 & -.. & --. \\
\hline B-1 & disp & R1R040 & U-71 & & $\mathrm{Al}-6$ & 5061 & & --. & & 7.960 & & 6.120 & 3.550 & 0.460 & -..- & -.- \\
\hline B-2 & disp & R2R040 & U-71 & & $\mathrm{Al}-2$ & $2 \mathrm{Si}$ & & -.- & & 7.950 & & 6.070 & 3.530 & 0.460 & -.. & --- \\
\hline B-3 & disp & R0R010 & U-71 & & A & $\mathrm{Al}$ & & ... & & 7.950 & & 6.080 & 3.530 & 0.448 & .-. & -.- \\
\hline B-4 & foil & H1T010 & U-12 & & . & - & & .254 & & 6.270 & & 5.520 & 3.210 & 0.750 & --- & --- \\
\hline B-5 & foil & L1FOIL & U-10 & & $\ldots$ & - & & .254 & & 5.900 & & 5.300 & 3.160 & 0.600 & -- & -- \\
\hline B-6 & disp & V5R050 & U-10 & & $\mathrm{Al}-0$ & $.5 \mathrm{Si}$ & & -- & & 8.150 & & 6.100 & 3.540 & 0.678 & -.. & --. \\
\hline B-7 & foil & L1F140 & U-10 & & & - & & .254 & & 6.520 & & 5.860 & 3.410 & 0.660 & -.- & -- \\
\hline B-8 & foil & MZ25 & U-71 & & ... & - & & 254 & & 6.350 & & 5.900 & 1.170 & 0.450 & -.- & --. \\
\hline C-1 & foil & H1F030 & U-12 & & - & - & & .254 & & 6.620 & & 5.820 & 3.380 & 0.800 & -.- & -- \\
\hline C-2 & foil & L1T020 & U-10 & & . & - & & .254 & & 6.390 & & 5.740 & 3.420 & 0.650 & .-. & --. \\
\hline C-3 & foil & L1F110 & U-10 & & . & . & & 254 & & 6.570 & & 5.910 & 3.440 & 0.660 & -.. & --- \\
\hline C-4 & foil & MZ50 & U-71 & & .. & -. & & .508 & & 11.900 & & 10.900 & 2.160 & 1.000 & ...- & -.- \\
\hline C-5 & foil & L1F120 & $\mathrm{U}-10$ & & - & - & & .254 & & 6.620 & & 5.960 & 3.470 & 0.660 & --. & --- \\
\hline C-6 & foil & H1T020 & U-12 & & $\ldots$ & - & & 254 & & 6.150 & & 5.400 & 3.190 & 0.750 & -..- & -.. \\
\hline C-7 & disp & R3R050 & U-7I & & $\mathrm{Al}-4$ & 4043 & & -- & & 7.970 & & 6.080 & 3.540 & 0.458 & -.. & -- \\
\hline C-8 & disp & R5R040 & U-71 & & $\mathrm{Al}-0$ & $.5 \mathrm{Si}$ & & .-. & & 7.96 & & 6.070 & 3.54 & 0.464 & -..- & -.- \\
\hline D-1 & disp & R1R050 & U-7I & & $\mathrm{Al}-6$ & 5061 & & -- & & 7.920 & & 6.080 & 3.540 & 0.460 & -.- & --- \\
\hline D-2 & blank & -..- & $\ldots$ & & - & - & & -.. & & -- & & -- & -.- & -.. & -.- & .-. \\
\hline D-3 & disp & R0R020 & U-71 & & A & $\mathrm{al}$ & & --- & & 7.950 & & 6.080 & 3.530 & 0.450 & -.- & --- \\
\hline D-4 & blank & --- & $\ldots$ & & . & - & & --. & & --- & & --- & -.. & --- & -.. & --. \\
\hline D-5 & blank & --- & .. & & -. & - & & --- & & -.. & & -.- & -.. & -.- & --. & --- \\
\hline D-6 & foil & L1F160 & U-10 & & .. & - & & .254 & & 5.41 & & 4.870 & 2.83 & 0.540 & -.- & --- \\
\hline D-7 & foil & L2F040 & U-10 & & .. & - & & .508 & & 12.040 & & 10.820 & 6.310 & 1.220 & -- & --. \\
\hline D-8 & disp & R2R050 & U-7N & & $\mathrm{Al}-\mathrm{Z}$ & $2 \mathrm{Si}$ & & -- & & 7.950 & & 6.070 & 3.530 & 0.462 & --- & --- \\
\hline & & otals: & & & & & & & & & & 56.670 & 84.930 & 15.458 & 0.000 & 0.000 \\
\hline & & Matrix Phase & & & & & & & & & & & Clad & Iding & & \\
\hline & & Mass (g) & & & Matt & rix Phas & se Cons & stituent $\mathrm{N}$ & Masses & $s(g)$ & & & Mas & $\mathrm{s}(\mathrm{g})$ & & \\
\hline & & & $\mathrm{Al}$ & $\mathrm{Si}$ & $\mathrm{Fe}$ & $\mathrm{Cu}$ & $\mathrm{Mn}$ & $\mathrm{Mg}$ & $\mathrm{Cr}$ & $\mathrm{Zn}$ & $\mathrm{Ti}$ & other & $\mathrm{Zr}$ & Al-6061 & & \\
\hline & & --- & -- & --- & --- & --- & --- & --- & --- & --- & -- & $-\cdots$ & & \begin{tabular}{|l|}
9.729 \\
\end{tabular} & & \\
\hline & & -- & -- & --- & --- & --- & --- & -.- & --. & -.- & --. & --- & & 9.729 & & \\
\hline & & --. & --. & -.- & --- & -.- & -- & -.- & -- & --. & --. & --. & & 9.729 & & \\
\hline & & ..- & -.- & --- & --- & -.- & -.- & .-. & --. & ..- & .-.- & ..- & & 9.729 & & \\
\hline & & 1.431 & 1.332 & 0.075 & 0.011 & 0.004 & 7E-04 & $7 \mathrm{E}-04$ & -.- & 0.001 & 0.003 & 0.002 & & 5.330 & & \\
\hline & & 1.369 & 1.3622 & 0.007 & -- & --- & --- & --- & -.- & --- & --- & --- & & 5.330 & & \\
\hline & & 1.422 & 1.4149 & 0.007 & -.- & --. & -.- & --. & -.- & --- & --. & -.- & & 5.330 & & \\
\hline & & -- & $-\cdots$ & -- & --- & --- & --. & -- & -.- & -.- & --. & --. & & 7.960 & & \\
\hline & & 1.380 & 1.330 & 0.008 & 0.010 & 0.004 & 0.002 & 0.014 & 0.005 & 0.003 & 0.002 & 0.002 & & 5.330 & & \\
\hline & & 1.420 & 1.392 & 0.028 & -- & $\cdots$ & $\cdots$ & $\cdots$ & $\cdots$ & $\cdots$ & $\cdots$ & $-\cdots$ & & 5.330 & & \\
\hline & & 1.422 & 1.31 & --- & -.- & --. & -..- & -..- & -..- & .-. & .-. & .-. & & 5.330 & & \\
\hline & & -.. & -.- & .-. & --. & .-. & -.. & -.. & --. & .-. & -.. & -.. & & 7.960 & & \\
\hline & & --. & ..- & -.- & -.- & .-- & -.-- & -.- & -.- & -.- & -.- & -.. & & 7.960 & & \\
\hline & & 1.372 & 1.3651 & 0.007 & --. & -.- & --. & -.- & --. & -.. & -.. & -.- & & 5.330 & & \\
\hline & & -.- & $-\cdots$ & --- & --- & --. & --. & --. & --. & -.- & -.. & -.- & & 7.960 & & \\
\hline & & --. & -.- & -.- & -.- & --. & -.- & --. & -.- & -.- & .-. & .-. & 19.369 & & & \\
\hline & & -.- & --. & --- & --- & --. & --. & --- & --. & -.. & -.- & -.. & & 7.960 & & \\
\hline & & -.- & -.- & -.- & --- & --- & --. & --- & --. & -.. & -.. & -.- & & 7.960 & & \\
\hline & & -- &.-- & -.. & -- & --. & -.. & -- & --. & -.- & --. & --. & & 7.960 & & \\
\hline & & -.- & -.- & -.- & --.- & .-- & -.- & -.- & -.- & -.- & -.- & -.- & 15.062 & & & \\
\hline & & -- & --. & --- & -- & --. & -- & -- & -- & -- & -- & -- & & 7.960 & & \\
\hline & & -.- & -.- & .-. & --- & --.- & .-. & --- & --.- & -.-- & -.- & -.- & & 7.960 & & \\
\hline & & 1.432 & 1.333 & 0.075 & 0.011 & 0.004 & 7E-04 & $7 \mathrm{E}-04$ & --. & 0.001 & 0.003 & 0.002 & & 5.330 & & \\
\hline & & 1.426 & 1.4189 & 0.007 & -- & --- & --- & -- & --- & --- & --- & --- & & 5.330 & & \\
\hline & & 1.380 & 1.330 & 0.008 & 0.010 & 0.004 & 0.002 & $\mid 0.014$ & 0.005 & 0.003 & 0.002 & 0.002 & & 5.330 & & \\
\hline & & -.- &.-- & --- & --. & 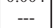 & --- & --- & --. & -- & --- & --- & & 9.729 & & \\
\hline & & 1.420 & 1.420 & -.- & --- & --- & --. & --- & --. & --- & --- & --. & & 5.330 & & \\
\hline & & -- & -- & --. & -- & --- & --. & --. & --. & --- & --. & --. & & 9.729 & & \\
\hline & & -.- & -.- & -.. &.-- & -.- & -..- &.-- & -.- & --. & --. & --. & & 9.729 & & \\
\hline & & -.. & -.. & ... &.- & ... &.- & -.. & $\ldots$ & -.. & -.. & -.- & & 7.960 & & \\
\hline & & -.- & -.- & --.- & --- & --- & --. & --- & --. & -.- & --. & -.- & & 6.190 & & \\
\hline & & 1.418 & 1.390 & 0.028 & -.- & .-- & ..- & .-- & $\ldots$ & .-- & -.- & --- & & 5.330 & & \\
\hline & & 16.892 & \begin{tabular}{|l|}
16.397 \\
\end{tabular} & \begin{tabular}{|l|}
0.252 \\
\end{tabular} & 0.042 & \begin{tabular}{|l|}
0.016 \\
\end{tabular} & \begin{tabular}{|l|}
0.006 \\
\end{tabular} & \begin{tabular}{|l|}
0.029 \\
\end{tabular} & 0.010 & 0.010 & \begin{tabular}{|l|}
0.010 \\
\end{tabular} & 0.008 & 34.431 & \begin{tabular}{|l|}
217.853 \\
\end{tabular} & & \\
\hline
\end{tabular}

Table 5. Constituent masses for each RERTR-7A mini-plate. 


\begin{tabular}{|c|c|c|c|c|c|c|c|c|c|c|c|c|c|c|c|c|c|c|}
\hline \multirow[b]{2}{*}{ Fuel Plate } & \multirow{2}{*}{$\begin{array}{l}\text { Fuel Phase } \\
\text { Composition }\end{array}$} & \multirow{2}{*}{$\begin{array}{l}\text { Fuel Meat } \\
\text { Volume }(\mathrm{cc})\end{array}$} & \multicolumn{5}{|c|}{ Fuel Phase Constituent Densities $(\mathrm{g} / \mathrm{cm} 3)$} & $\begin{array}{l}\text { Matrix Phase } \\
\text { Dens. }(\mathrm{g} / \mathrm{cm} 3)\end{array}$ & \multicolumn{10}{|c|}{ Matrix Phase Constituent Densities $(\mathrm{g} / \mathrm{cc})$} \\
\hline & & & Total U & $\mathrm{U}-235$ & Mo & $\mathrm{Si}$ & $\mathrm{Cr}$ & $\mathrm{Al}$ & $\mathrm{Al}$ & $\mathrm{Si}$ & $\mathrm{Fe}$ & $\mathrm{Cu}$ & $\mathrm{Mn}$ & $\mathrm{Mg}$ & $\mathrm{Cr}$ & $\mathrm{Zn}$ & $\mathrm{Ti}$ & other \\
\hline $6 \mathrm{AA}-1$ & --- & --- & --- & --- & --- & $-\overline{--}$ & --- & --- & --- & --- & --- & $\overline{---}$ & --- & --- & --- & --- & $\overline{---}$ & --- \\
\hline $6 \mathrm{~B}-5$ & --- & -- & -- & --- & --- & -- & -.- & --. & --. & --. & --- & -- & --- & --- & --- & --- & -- & --- \\
\hline $6 \mathrm{~A}-3$ & --- & --- & -- & --- & -.- & -.- & --- & --. & --- & --- & --- & --- & -- & --- & --- & --- & --- & --- \\
\hline $6 \mathrm{~A}-4$ & --- & --- & -- & --- & --- & --- & --. & -.- & --- & --- & --- & --- & --- & --- & --- & --- & --- & --- \\
\hline $6 \mathrm{~A}-5$ & U-7Mo & 0.957 & 6.332 & 3.689 & 0.480 & -- & --. & 1.495 & 1.392 & 0.079 & 0.012 & 0.004 & 0.001 & -- & -- & 0.001 & -- & 0.002 \\
\hline $6 \mathrm{~A}-6$ & U-10Mo & 0.957 & 6.405 & 3.710 & 0.712 & --- & --- & 1.431 & 1.423 & 0.007 & --- & --- & --- & --- & --- & --- & --- & --- \\
\hline $6 \mathrm{~A}-7$ & U-7Mo & 0.957 & 6.353 & 3.699 & 0.479 & --- & --. & 1.486 & 1.478 & 0.007 & --- & --- & --- & --- & --- & --- & --- & --- \\
\hline $6 \mathrm{~A}-8$ & U-12Mo & 0.397 & 14.223 & 8.383 & 1.964 & --- & --- & -- & --- & --- & --- & -.. & -.. & --- & --- & --. & -- & --- \\
\hline $6 \mathrm{~B}-1$ & U-7Mo & 0.957 & 6.395 & 3.710 & 0.481 & --- & --- & 1.442 & 1.389 & 0.009 & 0.010 & 0.004 & 0.002 & $\mid 0.014$ & 0.005 & 0.004 & 0.002 & 0.002 \\
\hline $6 \mathrm{~B}-2$ & U-7Mo & 0.957 & 6.343 & 3.689 & 0.481 & --- & --. & 1.484 & 1.454 & 0.030 & --- & --- & --- & --- & --- & --- & --- & --- \\
\hline $6 \mathrm{~B}-3$ & U-7Mo & 0.957 & 6.353 & 3.689 & 0.468 & -- & -- & 1.486 & 1.369 & -- & -- & -- & --- & -- & -- & -- & -- & -- \\
\hline 6B-4 & U-12Mo & 0.397 & 13.896 & 8.081 & 1.888 & --- & --. & -- & --- & --- & --- & --. & --- & --- & --- & --- & --- & --- \\
\hline 6B-5 & U-10Mo & 0.397 & 13.342 & 7.955 & 1.510 & --. & --. & -- & -.- & --. & --- & --. & -.. & --. & --- & --- & --. & --. \\
\hline 6B-6 & U-10Mo & 0.957 & 6.374 & 3.699 & 0.708 & -- & -- & 1.434 & 1.426 & 0.007 & --. & -- & -- & -- & -- & -- & -- & -- \\
\hline 6B-7 & U-10Mo & 0.397 & 14.752 & 8.584 & 1.662 & --- & --. & -- & --- & --- & --- & --- & --- & --- & --- & --- & --- & --- \\
\hline 6B-8 & U-7Mo & 0.397 & 14.853 & 2.945 & 1.133 & --- & --- & -- & --- & --- & --- & --- & --- & -- & --- & --- & --. & --- \\
\hline $6 \mathrm{C}-1$ & U-12Mo & 0.397 & 14.651 & 8.509 & 2.014 & --- & --- & -- & --- & --- & --- & -- & --- & --- & --- & --- & -- & --- \\
\hline $6 \mathrm{C}-2$ & U-10Mo & 0.397 & 14.450 & 8.610 & 1.636 & --- & --- & -- & --- & --- & --- & --- & --- & --- & --- & --- & --- & --- \\
\hline $6 \mathrm{C}-3$ & U-10Mo & 0.397 & 14.878 & 8.660 & 1.662 & -- & --- & -- & --- & --- & --. & --- & --- & -- & --- & --- & -- & --- \\
\hline $6 \mathrm{C}-4$ & U-7Mo & 0.794 & 13.720 & 2.719 & 1.259 & -- & --- & -- & -- & -- & --- & --- & -- & -- & --- & --- & -- & --- \\
\hline $6 \mathrm{C}-5$ & U-10Mo & 0.397 & 15.004 & 8.735 & 1.662 & --- & --. & -- & --- & --- & --- & --- & --- & --- & --- & --- & -- & --- \\
\hline $6 \mathrm{C}-6$ & U-12Mo & 0.397 & 13.594 & 8.031 & 1.888 & --- & --- & -- & -.- & --- & -.. & --- & --- & -.- & --- & --- & -.. & --- \\
\hline $6 \mathrm{~A}-7$ & U-7Mo & 0.957 & 6.353 & 3.699 & 0.479 & -- & --- & 1.496 & 1.393 & 0.079 & 0.012 & 0.004 & 0.001 & -- & --- & 0.001 & --- & 0.002 \\
\hline $6 C-8$ & U-7Mo & 0.957 & 6.343 & 3.699 & 0.485 & -- & --- & 1.490 & 1.483 & 0.007 & --- & --- & --- & --- & --- & -- & --- & --- \\
\hline $6 \mathrm{D}-1$ & U-7Mo & 0.957 & 6.353 & 3.699 & 0.481 & --. & --. & 1.442 & 1.389 & 0.009 & 0.010 & 0.004 & 0.002 & 0.014 & 0.005 & 0.004 & 0.002 & 0.002 \\
\hline $6 \mathrm{C}-3$ & --- & --- & --- & --- & --- & --- & --- & -- & --- & -- & --- & --- & -- & --- & --- & --- & --- & --- \\
\hline $6 \mathrm{D}-3$ & U-7Mo & 0.957 & 6.353 & 3.689 & 0.470 & --- & --. & 1.484 & 1.484 & --- & -- & -- & --. & --- & --- & --- & --- & --- \\
\hline $6 \mathrm{D}-4$ & --- & --- & -- & -- & -- & -- & --- & -- & --- & -- & --. & --- & -- & -- & --- & --- & -- & --- \\
\hline $6 \mathrm{~B}-5$ & --- & --- & -.- & --- & --- & --- & --. & -- & --- & --- & --. & --- & --- & --- & --- & --- & --- & --- \\
\hline $6 \mathrm{D}-6$ & U-10Mo & 0.397 & 12.260 & 7.124 & 1.359 & --. & $\cdots$ & -- & --- & --- & --- & --. & --- & -.- & --- & --- & --. & --. \\
\hline $6 \mathrm{D}-7$ & U-10Mo & 0.794 & 13.619 & 7.942 & 1.536 & --- & --- & -- & --- & --- & --- & --- & --- & --- & --- & --- & --. & --- \\
\hline $6 \mathrm{D}-8$ & U-7Mo & 0.957 & 6.343 & 3.689 & 0.483 & --- & --- & 1.482 & 1.452 & 0.030 & --- & --- & --- & --- & --- & --- & --- & --- \\
\hline
\end{tabular}

Table 6. Constituent densities for RERTR-7A mini-plates based on nominal fuel meat volume. 